\title{
Unprecedented Integral-Free Debye Temperature Formulas: Sample Applications to Heat Capacities of ZnSe and ZnTe
}

\author{
R. Pässler \\ Institut für Physik, Technische Universität Chemnitz, 09107 Chemnitz, Germany \\ Correspondence should be addressed to R. Pässler; passler@physik.tu-chemnitz.de
}

Received 19 March 2017; Accepted 30 April 2017; Published 18 September 2017

Academic Editor: Oleg Derzhko

Copyright (C) 2017 R. Pässler. This is an open access article distributed under the Creative Commons Attribution License, which permits unrestricted use, distribution, and reproduction in any medium, provided the original work is properly cited.

\begin{abstract}
Detailed analytical and numerical analyses are performed for combinations of several complementary sets of measured heat capacities, for ZnSe and ZnTe, from the liquid-helium region up to $600 \mathrm{~K}$. The isochoric (harmonic) parts of heat capacities, $C_{V h}(T)$, are described within the frame of a properly devised four-oscillator hybrid model. Additional anharmonicity-related terms are included for comprehensive numerical fittings of the isobaric heat capacities, $C_{p}(T)$. The contributions of Debye and nonDebye type due to the low-energy acoustical phonon sections are represented here for the first time by unprecedented, integralfree formulas. Indications for weak electronic contributions to the cryogenic heat capacities are found for both materials. A novel analytical framework has been constructed for high-accuracy evaluations of Debye function integrals via a couple of integralfree formulas, consisting of Debye's conventional low-temperature series expansion in combination with an unprecedented hightemperature series representation for reciprocal values of the Debye function. The zero-temperature limits of Debye temperatures have been detected from published low-temperature $C_{p}(T)$ data sets to be significantly lower than previously estimated, namely, $270( \pm 3) \mathrm{K}$ for ZnSe and $220( \pm 2) \mathrm{K}$ for ZnTe. The high-temperature limits of the "true" (harmonic lattice) Debye temperatures are found to be $317 \mathrm{~K}$ for $\mathrm{ZnSe}$ and $262 \mathrm{~K}$ for $\mathrm{ZnTe}$.
\end{abstract}

\section{Introduction}

Since the invocation of the concept of apparently characteristic, material-specific temperature parameters, $\Theta$, within Debye's classical paper [1] on specific heats of solids, one was concerned with a large variety of quotations of corresponding $\Theta_{D}$ values (so-called "Debye temperatures") within numerous thermophysical research papers, including various representative review articles [2-5] and books [6-10]. A brief inspection of the enormous $\Theta_{D}$ data material published hitherto, however, showed readily that it is obviously not possible to find unique $\Theta_{D}$ values for the individual materials in question, which should apply to duly broad temperature regions. In order to save, nevertheless, the apparently rather popular concept of Debye temperatures, it became thus already long ago a widely accepted custom to adopt the physically somewhat problematic notion of variable (T-dependent) Debye temperatures, $\Theta_{D}(T)$. Accordingly,
Debye's heat capacity model function [1] used to be employed in practice in the general form $[3,4,6,10-15]$

$$
\frac{C_{p}(T)}{C_{V h}(\infty)} \equiv \kappa(T)=\frac{3}{\left(x_{D}(T)\right)^{3}} \int_{0}^{x_{D}(T)} d x \frac{x^{4} e^{x}}{\left(e^{x}-1\right)^{2}} \leq 1,
$$

where the upper limits of integration are given by the ratios, $x_{D}(T)=\Theta_{D}(T) / T$, of adjustable Debye temperatures versus the respective lattice temperatures and $C_{V h}(\infty)$ represents the familiar Dulong-Petit limiting value for the isochoric lattice heat capacity in harmonic approximation (i.e., in particular $C_{V h}(\infty)=6 R$, for binary materials).

In the course of numerical fittings of measured (isobaric) heat capacities, $C_{p}(T)$, on the basis of (1), it has continually been found that, in contrast with Debye's original suggestion [1], proper simulations of such heat capacity curves can in fact be realized only by admitting rather strong $T$-dependencies of the material-specific Debye temperatures, $\Theta_{D}(T)$. This statement applies above all to the liquid-helium-hydrogen 
region, where the adjusted $\Theta_{D}(T)$ values are as a rule rapidly falling [3-5, 12-17] from their $T \rightarrow 0$ limiting levels, $\Theta_{D}(0)$, to certain minimum values, $\Theta_{D \min }$, which used to be located in the vicinities of $T \approx \Theta_{D}(0) / 15$. Furthermore, towards higher temperatures, many $\Theta_{D}(T)$ curves show a more or less pronounced increase up to certain material-specific maxima, $\Theta_{D \max }$, the actual magnitudes of which are in many cases significantly higher than the respective $\Theta_{D \min }$ levels. Such typical nonmonotonic behaviours of $\Theta_{D}(T)$ curves in the cryogenic region have been found in particular for $\mathrm{Si}$ and $\mathrm{Ge}$ [18-21] as well as for a large variety of III-V [11, 12, 15, 22-30], II-VI [14, 31-38], and I-VII [16, 39, 40] materials.

Globally one can thus assess that Debye's original idea, according to which heat capacities of solids might be represented by Debye function integrals (of type (1)), whose upper limits of integration should be based on fixed, materialspecific $\Theta_{D}$ values, is largely illusionary. Actually, not a single binary or ternary material could be found hitherto, within a wealth of thermophysical researches, for which it would have been possible to simulate in adequate way the temperature dependence of measured heat capacities, from the liquidhelium region up to the vicinity of room temperature, in terms of a unique $\Theta_{D}$ value. This means, among other things, that there is hardly a chance to come to appropriate quantitative descriptions of the temperature dependencies of heat capacities mainly via constructions of various, highly elaborate analytical models [41-44] for integrals of Debye's type and imputing, at the same time, only some fixed ( $T$ independent) values for Debye temperatures (like $\Theta_{D}=632 \mathrm{~K}$ for $\mathrm{GaN}$ [43] or $\Theta_{D}=920 \mathrm{~K}$ for $\mathrm{ZnO}[44]$ and $\Theta_{D}=946 \mathrm{~K}$ for $\mathrm{MgO}[44])$.

The basic cause of the occurrence of relatively deep minima, $\Theta_{D \min }$, in the cryogenic region had been indicated already many years ago by Schrödinger [2] to be obviously due to the onset of thermal activation of short-wavelength transverse acoustical (TA) phonons, which are quantumtheoretically manifested by pronounced peaks (singularities) in the respective material-specific phonon density of states (PDOS) spectra (see, e.g., the calculated $g_{P}(\varepsilon)$ spectra shown in $[45,46]$ for $\mathrm{ZnSe}$ and $\mathrm{ZnTe})$. Such drastic deviations of physically realistic PDOS spectra from Debye's naïve (quadratic) PDOS model function [1], $g_{D}(\varepsilon) \propto \varepsilon^{2}$, are continually confirmed to be indeed the main cause of the obviously typical, nonmonotonic (non-Debye) behaviours of $\Theta_{D}(T)$ curves.

Of course, when a representative set of properly determined $\Theta_{D}(T)$ values is actually available for one or the other material in question, one can readily calculate the respective heat capacities, either via direct numerical evaluations of the integral occurring in (1) or by interpolations between discrete $\widehat{x}_{D}$ values quoted in some of the more or less comprehensive $C\left(\widehat{x}_{D}\right)$ or $C\left(\widehat{x}_{D}\right) / C_{V h}(\infty)$ tables presented by various authors $[6,8,47-49]$ (see also the list of presently obtained highaccuracy sample values given in Table 7).

Considerably more problematic and difficult, however, is a proper computational solution of the hitherto largely ignored inverse problem, namely, the reliable determination of effective Debye temperatures, $\Theta_{D}(T)$, on the basis of measured heat capacities, $C_{p}(T)$. In view of the longstanding absence of corresponding integral-free formulas, it was the custom in thermophysical investigations over a long time to resort to some of the tables quoted above and to perform thus approximate estimations of the respective $x_{D}$-ratios via interpolations between discrete $\widehat{x}_{D}$-ratios quoted for the couples of the two neighbouring $C_{p}(\widehat{T})\left(\right.$ or $\left.C_{p}(\widehat{T}) / C_{V h}(\infty)\right)$ values. Such conventional interpolation procedures were still feasible as long as one used to be concerned only with relative small sets (limited to few dozens) of measured $C_{p}(T)$ data points. However, in contemporary literature, one is repeatedly concerned with rather large sets (of hundreds) of $C_{p}(T)$ data points (like the presently analyzed $C_{p}(T)$ data sets given in [46] for ZnSe and ZnTe). With respect to the latter such clumsy point-by-point interpolation procedures appear to be largely obsolete. A practicable computational alternative, however, requires the availability of a duly elaborate analytical framework for the inverse relationship, which should hence enable performing straightforward evaluations of the actual $x_{D}(T)=\Theta_{D}(T) / T$-ratios directly on the basis of given heat capacity values, $C_{p}(T)$. It is the main purpose of the present study to develop here (within Appendix B) such a representative analytical framework of integral-free formulas and to present corresponding sample results for Debye temperatures of $\mathrm{ZnSe}$ and $\mathrm{ZnTe}$.

Concerning the successive development of this novel analytical apparatus for Debye temperatures, we would still like to note that some partial results in form of asymptotic (approximate) Debye temperature expressions had already been published in two preceding papers, namely, in [13] for the low-temperature region $\left(T<\Theta_{D}(T) / 12\right)$,

$$
\Theta_{D}(T) \longrightarrow \Theta_{D}^{l_{0}}(T)=T \cdot\left(\frac{4 \pi^{4} C_{V h}(\infty)}{5 C_{p}(T)}\right)^{1 / 3},
$$

and in [15] for the complementary range of higher temperatures $\left(T>\Theta_{D}(T) / 12\right)$,

$$
\begin{aligned}
\Theta_{D}(T) & \approx \Theta_{D}^{h_{0}}(T) \\
& =T \cdot \sqrt[2]{-35+5 \cdot \sqrt[2]{49+56\left(\frac{C_{V h}(\infty)}{C_{p}(T)}-1\right)} .} .
\end{aligned}
$$

However, due to a lack of space, the somewhat unusual derivation procedure for this highly useful high-temperature formula (i.e., Eq. (26) in [15]) could not be included into the former paper. A duly detailed display of the corresponding analytical derivation procedure is now made up, among other things, in Appendix B.

In Section 2 we briefly sketch the basic arguments and equations of the analytical framework for theoretical calculations of isobaric heat capacities. In Section 3 we display a reformulated, integral-free version of the multioscillator hybrid model $[14,21,50]$. This is based mainly on the usage of novel, unprecedented analytical formulas (derived in Appendix A) for the contributions of the continuous, longwavelength sections of transverse acoustical (TA) phonons to 
the resulting lattice heat capacities. In Section 4 we adopt (in analogy to [14]) a special 4-peak Einstein oscillator constellation of the hybrid model, and we use the respective analytical heat capacity expression for careful fittings of compatible sets of low- and high-temperature data available for $\mathrm{ZnSe}$ and ZnTe (up to $600 \mathrm{~K}$ ). In Section 5 we perform, on the basis of a couple of precision formulas derived in Appendix B, transformations of the experimental heat capacity data in question (including the corresponding theoretical $C_{p}(T)$ and $C_{V h}(T)$ curves), into the respective Debye temperature representations. The results are discussed in Section 6.

\section{Basic Equations for Temperature Dependencies of Heat Capacities in Solids}

Within the frame of the harmonic lattice regime, the temperature dependencies of the isochoric heat capacities, $C_{V h}(T)$, are well known to be given by corresponding heat capacity shape functions, $\kappa_{h}(T)$, of the general form $[4,6,45,50-55]$

$$
\begin{aligned}
\frac{C_{V h}(T)}{C_{V h}(\infty)} & \equiv \kappa_{h}(T)=\int d \varepsilon g_{P}(\varepsilon)\left(\frac{\varepsilon / 2 k_{B} T}{\sinh \left(\varepsilon / 2 k_{B} T\right)}\right)^{2} \\
& \leq 1,
\end{aligned}
$$

where we have denoted by $\varepsilon=\hbar \omega$ the energies of the individual phonons and $g_{P}(\varepsilon)$ represents the material-specific PDOS spectral function $[3,4,19,56]$. The latter is supposed within the present context to have been normalized to unity $[6,45,52,53,55]$; that is, $\int d \varepsilon g_{P}(\varepsilon)=1$.

Within the frame of numerical analyses of experimental heat capacity data, however, one is continually concerned with a basic theoretical complication due to the inherent differences between the temperature dependencies of theoretical $C_{V h}(T)$ functions, on the one hand, and those of measured (isobaric) heat capacities, $C_{p}(T)$, on the other hand. The latter are well known to be throughout somewhat higher, $C_{p}(T)>C_{V h}(T)$, than their theoretical (isochoric) counterparts pertaining to the harmonic lattice regime $[4,50$, 54]. The respective differences, $C_{p}(T)-C_{V h}(T)>0$, are usually found to be very small from 0 up to temperatures of order $T_{h}$, where the heat capacity amounts to about $50 \%$ of the Delong-Petit limiting value, $C_{p}\left(T_{h}\right) \cong C_{V h}\left(T_{h}\right)=$ $C_{V h}(\infty) / 2$. On the other hand, one is usually concerned with a relatively strong monotonic increase of these differences, $d\left(C_{p}(T)-C_{V h}(T)\right) / d T>0$, at higher temperatures $(T>$ $T_{h}$ ). These differences are generally ascribed to cumulative effects of lattice expansion and lattice anharmonicities [3, $11,18,21,23,39,50,54,56,57]$. We have repeatedly found within a larger series of heat capacity studies $[14,15,21,50,54]$ that, within regions from $T_{h}$ up to temperatures of order $5 T_{h}$ (at least), the rapid increase of these differences can be simulated in good approximation by a proportionality of type $C_{p}(T)-C_{V h}(T) \propto T \times\left(C_{V h}(T)\right)^{2} \propto T \times\left(\kappa_{h}(T)\right)^{2}$ (note that the structure of the latter is analogous to the known Nernst-Lindemann formula $[2,3,58], C_{p}(T)-C_{V}(T) \propto T \times$ $\left(C_{p}(T)\right)^{2}$, for the differences between isobaric and isochoric heat capacities). Admitting, furthermore, that at sufficiently high temperatures (up to melting points, if necessary) some additional higher-order power terms of $T$ may come into play, it appeared reasonable to simulate the $T$-dependencies of isobaric heat capacities by a duly general algebraic expression of the form $[14,15,21,50,54,59]$

$$
\begin{aligned}
C_{p}(T)= & C_{V h}(\infty)\left[\kappa_{h}(T)+\left(\kappa_{h}(T)\right)^{2} \sum_{n=1}^{(\infty)} A_{n} T^{n}\right] \\
& +c_{1} T,
\end{aligned}
$$

where the empirical Taylor series expansion coefficients, $A_{n}(n=1,2, \ldots)$, are quantifying the material-specific weights of contributions of the individual, anharmonicityrelated $T^{n}$ power terms $[15,21,54,57]$. Furthermore we have still included into (5) an additional linear term, $C^{(E)}(T)=c_{1} T$, which has to represent possible contributions of a degenerate electronic system $[4,6,13,23,60-63]$ to the measured heat capacities. Such electronic contributions ("Sommerfeld terms" $[4,6,60])$ are known to be rather strong (tending to become even the dominant ones, at sufficiently low temperatures) in metals, due to large concentrations of conduction electrons. In contrast to this, such electronic contributions are as a rule rather weak in semiconductors and insulators due to the relatively low concentrations of free electrons associated with lattice imperfections (impurities). Accordingly, the actual strengths of such electronic contributions can vary considerably even between different samples of one and the same semiconductor material $[4,62]$. We will find below (in Section 4) indications for some weak electronic contributions to the isobaric heat capacities measured at liquid-helium temperatures [46], for both $\mathrm{ZnSe}$ and $\mathrm{ZnTe}$.

\section{Multioscillator Hybrid Model and Its Integral-Free Representation}

Physically realistic, material-specific phonon density of states (PDOS) functions, $g_{P}(\varepsilon)$ (like those shown, e.g., in Figure 3 of [45] or in Figure 4 of [46], for ZnSe and ZnTe), which are determining the $T$-dependencies of the respective harmonic heat capacity shape functions, $\kappa_{h}(T)$ (see (4)), are known to consist in general of numerous peaks (critical points) in combination with various continuous curve sections and/or gaps between them. Many fine details of such materialspecific PDOS spectra, however, are practically not detectible via analyses of experimental heat capacity data $[3,64]$. Their possible influence on thermal properties used to be strongly reduced by the thermal averaging process (in (4)), so that they are not discernable from the background of accidental experimental uncertainties (being usually of the order $\pm 1 \%$ ). Consequently, it is as a rule possible to realize rather good fittings of available $C_{p}(T)$ data on the basis of relatively simple theoretical model functions for PDOS spectra [64], provided that the latter are sufficiently general and flexible for reasonable reproductions of various prominent features, at least. This concerns above all the liquid-helium-hydrogen region $[13,64]$, where the lattice heat capacities are known to be represented in good approximation by Taylor series of odd-order terms $[13,16,18,23,35,39,65]$

$$
C_{p}^{(L)}(T) \longrightarrow C_{V h}(T)=c_{3} T^{3}+c_{5} T^{5}+\cdots .
$$


This limiting $C_{V h}(T)$ behaviour is the automatic consequence of the well-known fact that the low-energy (TA phonon) tails of PDOS spectra for three-dimensional crystals are generally given by Taylor series $[13,16,18,23,35,39$, $64,65]$ of exclusively even-order power terms, $g_{P}^{\text {(cont.) }}(\varepsilon)=$ $\gamma_{2} \varepsilon^{2}+\gamma_{4} \varepsilon^{4}+\cdots$, of the phonon energy. An incorporation of a corresponding continuous low-energy section into theoretical $g_{p}(\varepsilon)$ model functions is therefore indispensable for their actual applicability to the cryogenic region. At the same time it is also clear that such continuous low-energy tail sections are naturally ending (i.e., $g_{P}^{\text {(cont.) }}\left(\varepsilon \geq \varepsilon_{1}\right)=0$ ) at the positions of the first critical points $[14,21,50,64], \varepsilon_{1}$, which are usually corresponding to the first peaks of shortwavelength TA phonons (note that, for materials with zinc blende structure like $\mathrm{ZnSe}$ and $\mathrm{ZnTe}$, the energy positions of the first critical points are corresponding to those of the Lpoints on the first Brillouin zone boundaries, i.e., $\varepsilon_{1}=\varepsilon_{\mathrm{TA}}(L)$ $[7,32,36,45,46,66,67])$.

\subsection{General Analytical Framework of Multioscillator Hybrid} Models. Less critical than the general ansatz for the $0<\varepsilon<\varepsilon_{1}$ region is the modelling of $g_{P}(\varepsilon)$ spectra for energies higher than the first critical points, $\varepsilon>\varepsilon_{1}$. Numerous analyses have shown that excellent simulations of $C_{V h}(T) \propto \kappa_{h}(T)$ dependencies (4), from cryogenic to room temperatures, can be realized on the basis of combinations of several (macroscopic) Einstein oscillators [68], $\propto w_{n} \delta\left(\varepsilon-\varepsilon_{n}\right)\left(n=1\right.$ to $\left.n_{E}\right)$, the ordered energy positions of which, $\varepsilon_{1}<\varepsilon_{2}<\cdots<\varepsilon_{n_{E}}$, including their actual weights, $w_{n}$, are to be properly adjusted in the course of least-mean-square fitting processes for heat capacities. Thus one can take the normalized PDOS function in (4) to be generally representable by a multioscillator hybrid model function of the sufficiently general form $[14,21,50]$

$$
\begin{aligned}
g_{P}(\varepsilon)= & \vartheta\left(\varepsilon_{1}-\varepsilon\right) \sum_{k=1}^{2} w_{C k} \frac{(2 k+1) \varepsilon^{2 k}}{\varepsilon_{1}^{2 k+1}} \\
& +\sum_{n=1}^{n_{E}} w_{n} \delta\left(\varepsilon-\varepsilon_{n}\right)
\end{aligned}
$$

where we have denoted by $\vartheta\left(\varepsilon_{1}-\varepsilon\right)\left(=1\right.$ for $\varepsilon<\varepsilon_{1}$ and 0 for $\varepsilon>$ $\varepsilon_{1}$ ) the step function, which realizes the cut of the continuous part of the $g_{P}(\varepsilon)$ function just at the first singular point. By integrating (7) over the whole spectrum (from 0 beyond the highest Einstein oscillator), we see that the supposed normalization of the $g_{P}(\varepsilon)$ function to unity is equivalent to a normalization condition of the form $[14,21,50]$

$$
1=\int_{0}^{(\rightarrow \infty)} d \varepsilon g_{P}(\varepsilon)=\sum_{k=1}^{2} w_{C k}+\sum_{n=1}^{n_{E}} w_{n}
$$

for the whole set of weighting factors. This condition is to be strictly fulfilled within any fitting process of experimental data on the basis of the $g_{P}(\varepsilon)$ model function (7).

Inserting, finally, the $g_{P}(\varepsilon)$ function (see (7)) into the integral (4) and denoting by $\Theta_{n} \equiv \varepsilon_{n} / k_{B}\left(n=1\right.$ to $\left.n_{E}\right)$ the characteristic Einstein temperatures corresponding to the energy positions of the individual macroscopic oscillators we obtain for the normalized (harmonic) heat capacity shape function the general expression $[14,21,50]$

$$
\begin{aligned}
\kappa_{h}(T)= & \sum_{k=1}^{2} w_{C k} \kappa_{C k}\left(x_{1}(T)\right) \\
& +\sum_{n=1}^{n_{E}} w_{n}\left(\frac{\Theta_{n} / 2 T}{\sinh \left(\Theta_{n} / 2 T\right)}\right)^{2},
\end{aligned}
$$

where the $\kappa_{C k}\left(x_{1}(T)\right)$ functions (being due to the two continuous components, $\propto \varepsilon^{2 k}$, in (7)) are given in form of integrals of Debye and non-Debye type $[14,21]$

$$
\kappa_{C k}\left(x_{1}(T)\right)=\frac{2 k+1}{\left(x_{1}(T)\right)^{2 k+1}} \int_{0}^{x_{1}(T)} d x \frac{x^{2 k+2} e^{x}}{\left(e^{x}-1\right)^{2}} .
$$

Here we have denoted by

$$
x_{1}(T) \equiv \frac{\Theta_{1}}{T}
$$

the ratio of the characteristic phonon temperature associated with the first (lowest) special point, $\Theta_{1}$, versus lattice temperature, $T$ (in analogy with the notation used within Debye's theory, $x_{D}(T) \equiv \Theta_{D} / T$; cf. (1) and Appendix A).

Informative byproducts of numerical fittings of measured $C_{p}(T)$ data sets via (5), in combinations with (9) for the harmonic heat capacity shape function, $x_{h}(T)$ (including (10), for the $\kappa_{C k}\left(x_{1}(T)\right)$ components), are the moments, $\mu_{P}^{(m)}$, of material-specific PDOS spectral functions $[3,4,16,18,19,21$, $50,52,56,69-72]$, which are generally defined by

$$
\mu_{P}^{(m)} \equiv \int d \varepsilon g_{P}(\varepsilon) \varepsilon^{m}, \quad \text { for }-3<m \neq 0
$$

Inserting the $g_{P}(\varepsilon)$ hybrid model function (see (7)) into the integral for $\mu_{P}^{(m)}$ (see (12)), we obtain for these moments the model-specific expression $[14,21]$

$$
\mu_{P}^{(m)}=\left(\frac{3 w_{C 1}}{3+m}+\frac{5 w_{C 2}}{5+m}\right) \varepsilon_{1}^{m}+\sum_{n=1}^{n_{E}} w_{n} \varepsilon_{n}^{m} .
$$

3.2. Integral-Free Formulas for the Contributions of LongWave Acoustical (TA) Phonons. On the basis of the hitherto used original (i.e., integral) expressions (see (10)) for the two low-phonon-energy contributions, $\kappa_{C 1 / C 2}\left(x_{1}(T)\right)$ (in (9)), the corresponding least-mean-square analysis procedures performed in several preceding studies $[14,21,50]$ turned out to be somewhat heavy and time-consuming. This disadvantage of the former hybrid model versions [14, 21, 50] was one of the main motivations for performing here (in Appendix A) a comprehensive analytical study aiming at the construction of more practicable, integral-free formulas for the integrals (10) of Debye and non-Debye type.

Consider first the integral (10) for the low-energy component of Debye type, $\kappa_{C k=1}\left(x_{1}(T)\right)$ (see Figure 1). A detailed analyses (in Section A.2 of Appendix A) has shown that the corresponding $\kappa_{C 1}\left(x_{1}\right)$ dependence (see (A.13)) can be 
represented in good approximation in terms of a proper combination (A.16) of truncated low- and high-temperature expansions, $\kappa_{C 1 l}^{n_{D}=1}\left(x_{1} \geq x_{c 1(D)}\right)\left(\right.$ see (A.14)) and $\kappa_{\mathrm{C} 1 h}^{m_{R}=7}\left(x_{1} \leq\right.$ $x_{c 1(D)}$ ) (see (A.15)), assuming a switch between the two complementary branches just at their crossing point, $x_{c 1(D)}$ $=6.5135$ (cf. the upper inset of Figure 1). This means for the low-temperature branch, $x_{1}(T) \equiv \Theta_{1} / T \geq x_{c 1(D)}=6.5135$,

$$
\begin{aligned}
\kappa_{C 1}\left(x_{1}\right) \longrightarrow & \kappa_{C 1 l}^{n_{D}=1}\left(x_{1}\right) \\
= & \frac{4 \pi^{4}}{5 x_{1}^{3}}-\frac{3 x_{1}}{e^{x_{1}}-1} \\
& -12 e^{-x_{1}}\left(1+\frac{3}{x_{1}}+\frac{6}{x_{1}^{2}}+\frac{6}{x_{1}^{3}}\right),
\end{aligned}
$$

and for the high-temperature branch, $0 \leq x_{1}(T) \equiv \Theta_{1} / T \leq$ $x_{c 1(D)}=6.5135$,

$$
\kappa_{C 1}\left(x_{1}\right) \longrightarrow \kappa_{C 1 h}^{m_{R}=7}\left(x_{1}\right)=\left[1+\sum_{m=1}^{m_{R}=7} c_{2 m}^{D R} x_{1}^{2 m}\right]^{-1}
$$

(the respective expansion coefficients of which, $c_{2 m}^{D R}$, are listed in Table 6).

Concerning the integral (10) for the second low-energy component, $\kappa_{C k=2}\left(x_{1}(T)\right)$ (see Figure 1), we have found within the frame of a detailed study (in Section A.2, of Appendix A) that the corresponding $\kappa_{C 2}\left(x_{1}\right)$ dependence of non-Debye type (see (A.17)) can be represented in good approximation in terms of the combination (A.24) of the truncated low- and high-temperature expansions, $\kappa_{\mathrm{C} 2 l}^{n_{N}=1}\left(x_{1} \geq x_{c 2(N)}\right)$ (see (A.18)) and $\kappa_{C 2 h}^{m_{R}=6}\left(x_{1} \leq x_{c 2(N)}\right)$ (see (A.23)), assuming again a switch between the two complementary branches at the respective crossing point, $x_{c 2(N)}=7.0962$ (cf. the lower inset of Figure 1). This means for the low-temperature branch, $x_{1}(T) \equiv \Theta_{1} / T \geq$ $x_{c 2(N)}=7.0962$, explicitly

$$
\begin{aligned}
\kappa_{C 2}( & \left.x_{1}\right) \longrightarrow \kappa_{C 2 l}^{n_{N}=1} \\
= & \frac{80 \pi^{6}}{21 x_{1}^{5}}-\frac{5 x_{1}}{e^{x_{1}}-1} \\
& -30 e^{-x_{1}}\left(1+\frac{5}{x_{1}}+\frac{20}{x_{1}^{2}}+\frac{60}{x_{1}^{3}}+\frac{120}{x_{1}^{4}}+\frac{120}{x_{1}^{5}}\right),
\end{aligned}
$$

and for the high-temperature branch, $0 \leq x_{1}(T) \equiv \Theta_{1} / T \leq$ $x_{c 2(N)}=7.0962$,

$$
\kappa_{\mathrm{C} 2}\left(x_{1}\right) \longrightarrow \kappa_{\mathrm{C} 2 h}^{m_{R}=6}\left(x_{1}\right)=\left[1+\sum_{m=1}^{m_{R}=6} c_{2 m}^{N R} x_{1}^{2 m}\right]^{-1}
$$

(the respective expansion coefficients of which, $c_{2 m}^{N R}$, are listed in Table 6).

The large scale representations of the $\kappa_{C 1}\left(x_{1}\right)$ and $\kappa_{C 2}\left(x_{1}\right)$ curves in Figure 1 are suggesting apparently perfect coincidences between the exact (solid) curves and the respective approximate curve sections. At the same time we see, from



FIGURE 1: Graphical representation of the exact $x_{1}$-dependencies (-) of the normalized low-phonon-energy contributions to lattice heat capacities of Debye and non-Debye type, $\kappa_{\mathrm{C} 1}\left(x_{1}\right)$ and $\kappa_{\mathrm{C} 2}\left(x_{1}\right)$ (see (10)), in comparison with the respective low-temperature approximations (--.---·), due to the functions $\kappa_{C 1 l}^{n_{D}=1}\left(x_{1} \geq x_{c 1(D)}\right)$ (see (14)) or $\kappa_{C 2 l}^{n_{N}=1}\left(x_{1} \geq x_{c 2(D)}\right)$ (see (16)), the associated high-temperature approximations $(-----)$, due to the functions $\kappa_{C 1 h}^{m_{R}=7}\left(x_{1} \leq x_{c 1(D)}\right)($ see $(15))$ or $\kappa_{C 2 h}^{m_{R}=6}\left(x_{1} \leq x_{c 2(D)}\right)$ (see (17)), and the normalized heat capacity contribution of the first Einstein oscillator $(-\cdots-), \kappa_{E 1}\left(x_{1}\right)=\left(\left(x_{1} / 2\right) / \sinh \left(x_{1} / 2\right)\right)^{2}$.

the insets of Figure 1, that the approaches to the exact $\kappa_{C k}\left(x_{1}\right)$ functions are really excellent only in the regions $x_{1}<5$ and $x_{1}>9$, whereas one is concerned in the vicinities of the crossing points, that is, at $x_{1} \approx x_{c 1(D)}=6.5135$, for the Debye-like component, and at $x_{1} \approx x_{c 2(N)}=7.0962$, for the non-Debye component, with certain overestimations of the exact $\kappa_{C k}\left(x_{1}\right)$ values by the approximate formulas (up to a maximum order of $0.008 \%$, for $k=1$, or of $0.015 \%$, for $k=2$ ). Yet, such computational inaccuracies are by 1 to 2 orders of magnitude smaller than the typical uncertainties of measured heat capacities, $C_{p}(T)$. Thus, these computational inaccuracies are usually negligible within the frame of practical applications.

3.3. Connection with Conventional Cryogenic Heat Capacity Parameters. At sufficiently low (liquid-helium) temperatures, where $x_{1}(T) \equiv \Theta_{1} / T>12$, the first terms $\left(\propto x_{1}^{-3}\right.$ or $\propto x_{1}^{-5}$ ) in the respective low-temperature expressions (see (14) and (16)) are strongly dominating. Thus the Debye-like and non-Debye components, $\kappa_{C k}\left(x_{1}(T)\right)$ ((see (10) for $k=$ 1 and 2 , resp.), tend here to the known $\propto T^{3}$ and $\propto T^{5}$ asymptotes $[14,21]$

$$
\begin{aligned}
& \kappa_{C 1}\left(x_{1}(T)\right) \longrightarrow\left(\frac{4 \pi^{4}}{5}\right) \cdot\left(\frac{T}{\Theta_{1}}\right)^{3}, \\
& \kappa_{C 2}\left(x_{1}(T)\right) \longrightarrow\left(\frac{80 \pi^{6}}{21}\right) \cdot\left(\frac{T}{\Theta_{1}}\right)^{5} .
\end{aligned}
$$

The asymptote of the $k=1$ component is of Debye type [1], whereas the asymptote of the $k=2$ component is 
responsible for the onset of the typical non-Debye behaviour. The latter is frequently emerging already at very low temperatures (of order $T \approx \Theta_{D}(0) / 50$ ), where the Einstein oscillator terms in (9) for the $\kappa_{h}(T)$ dependence are practically negligible (owing to their plateau behaviour, $\left(\Theta_{n} /\left(2 T \sinh \left(\Theta_{n}\right)\right.\right.$ $2 T)))^{2} \rightarrow\left(\Theta_{n} / T\right)^{2} \exp \left(-\Theta_{n} / T\right) \rightarrow 0$; cf. also the $\kappa_{E 1}\left(x_{1}\right)$ curve in Figure 1). In this way we satisfy ourselves that the low-temperature dependence of the harmonic lattice heat capacity, $C_{V h}(T)=C_{V h}(\infty) \kappa_{h}(T)$ (see (4)), reduces, as expected, to a couple of two odd-order terms $[13,16,18,23$, $35,39,65], C_{V h}(T) \rightarrow c_{3} T^{3}+c_{5} T^{5}$ (see (6)). The respective expansion coefficients, $c_{3}$ and $c_{5}$, are readily seen to be given in terms of the weighting factors $w_{\mathrm{C} 1}$ and $w_{\mathrm{C} 2}$, the characteristic phonon temperature associated with the first Einstein peak, $\Theta_{1} \equiv \varepsilon_{1} / k_{B}$, and the classical Dulong-Petit heat capacity value, $C_{V h}(\infty)$, by the relations

$$
\begin{aligned}
& c_{3}=w_{C 1} \frac{4 \pi^{4}}{5 \Theta_{1}^{3}} C_{V h}(\infty), \\
& c_{5}=w_{C 2} \frac{80 \pi^{6}}{27 \Theta_{1}^{5}} C_{V h}(\infty) .
\end{aligned}
$$

Comparing, finally, the analytical expression for the limiting (cubic) asymptote implied by the present formalism, $C_{V h}(T) \rightarrow c_{3} T^{3}=w_{C 1}\left(4 \pi^{4} / 5\right)\left(T / \Theta_{1}\right)^{3} C_{V h}(\infty)$ (due to (19)), with its conventional predecessor, $C_{V h}(T) \rightarrow\left(4 \pi^{4} / 5\right)(T /$ $\left.\Theta_{D}(0)\right)^{3} C_{V h}(\infty)$ (due to Debye's theory $[1-3,8,13]$ ), we see that these two $C_{V h}(T \rightarrow 0)$ expressions are coincident when the weighting factor for the Debye-like component, $w_{\mathrm{Cl}}$, is just given by the cube $[14,21]$ of the ratio between the characteristic phonon temperature associated with the first Einstein peak, $\Theta_{1}$, and the $T \rightarrow 0$ limit value, $\Theta_{D}(0)$ of the conventional Debye temperature; that is,

$$
w_{C 1}=\left(\frac{\Theta_{1}}{\Theta_{D}(0)}\right)^{3} .
$$

\section{Sample Analyses of Heat Capacity Data Sets for $\mathrm{ZnS}$ and $\mathrm{ZnTe}$}

For the sake of numerical analyses of given experimental heat capacity data within the frame of a multioscillator hybrid model like that displayed above, it is still necessary to specify the material-specific constellation (in particular the total number, $n_{E}$ ) of Einstein oscillators to be taken into consideration. A relatively simple version in form of a threeoscillator-based hybrid model [21] had been used in [50] for a large variety of III-V and II-VI materials with partly different crystal structures. Within the present (more detailed) study, for $\mathrm{ZnSe}$ and $\mathrm{ZnTe}$, we shall consider a structure-specific constellation of four discrete oscillators (in analogy to [14]), which offers a physically adequate association with the contributions of the 6 phonon branches in zinc blende structure materials (consisting of 2 TA branches and $1 \mathrm{LA}$ branch, for the lower parts, and 2 TO branches and $1 \mathrm{LO}$ branch, for the upper parts, of the respective PDOS spectra [7, 32, 36, 45, 46, $66,67])$. Let us briefly sketch the basic arguments in favour of the corresponding four-oscillator model [14].
4.1. Four-Oscillator Constellation Chosen for ZnSe and ZnTe. In the course of numerous analyses of experimental $C_{p}(T)$ data sets by different versions of the multioscillator hybrid model $[14,21,50]$ we have repeatedly made the experience that a necessary condition for the contributions of two oscillator peaks to be actually discernible, in the course of leastmean-square fitting processes, consists in a sufficiently large distance (of more than $10 \%$, at least) between their respective $\left(\varepsilon_{n+1}\right.$ versus $\left.\varepsilon_{n}\right)$ energy positions. Considering under this aspect the known positions of the dominating optical $\left(\varepsilon_{\mathrm{LO}}\right.$ versus $\left.\varepsilon_{\mathrm{TO}}\right)$ phonon peaks $[7,32,36,45,46,66,67]$ in $\mathrm{ZnSe}$ and $\mathrm{ZnTe}$ we see that their relative distances are markedly smaller than $10 \%$, so that their individual contributions to the measured $C_{p}(T)$ dependencies are practically indiscernible. Consequently, the contributions of the three optical phonon branches can be lumped together, being thus representable within the four-oscillator hybrid model [14] by a single oscillator with the highest energy, $\varepsilon_{4} \equiv k_{B} \Theta_{4}=k_{B} \Theta_{\text {TO\&LO }}$, and a fixed weight of

$$
w_{4}=w_{\text {TO\&LO }}=\frac{1}{2} .
$$

Qualitatively quite different is the state of affairs for the three acoustical phonon branches. From the theoretically calculated PDOS spectra [7, 32, 36, 45, 46, 66, 67] one can see that the distances of the dominating short-wave LA peaks from the prominent TA peaks (as well as from LO/TO peaks) are larger than $30 \%$. The contributions of the dominating LA peaks, $\varepsilon_{3} \equiv k_{B} \Theta_{3}=k_{B} \Theta_{\mathrm{LA}}$ (= centres of gravity of the LA branches), are thus well discernible from the contributions of TA phonons as well as from those of the LO/TO phonons. This corresponds in reasonable approximation to a fixed weight of

$$
w_{3}=w_{\mathrm{LA}}=\frac{1}{6}
$$

for the $\varepsilon_{3}$-oscillator. Finally, with respect to the 2 TA phonon branches, we observe that the energy differences, for example, between the special points $\varepsilon_{\mathrm{TA}}(L)$ and $\varepsilon_{\mathrm{TA}}(X)$, are larger than $20 \%[7,32,36,45,46,66,67]$, so that their individual contributions have good chances to be still discernible within the frame of duly careful numerical analysis procedures. This observation suggests representing the whole variety of shortwave TA phonons by two discrete model oscillators, $\varepsilon_{n} \equiv$ $k_{B} \Theta_{n}=k_{B} \Theta_{\mathrm{TA} n}(n=1$ and 2$)$. Concerning their actual weights, $w_{1}$ and $w_{2}$, we observe that, owing to the preceding fixations of the weights for the LA and LO/TO oscillators (i.e., $w_{4}=1 / 2(21)$ and $\left.w_{3}=1 / 6(22)\right)$, the global normalization condition (8) for the weights of all contributions associated with the two TA phonon branches (i.e., $w_{1}$ and $w_{2}$, in combination with $w_{\mathrm{C} 1}$ and $w_{\mathrm{C} 2}$ ) reduces, consequently, to a normalization condition of the TA phonon-related weighting factors of the special form [14]

$$
w_{\mathrm{C} 1}+w_{\mathrm{C} 2}+w_{1}+w_{2}=w_{\mathrm{TA}}=\frac{1}{3} .
$$

Accordingly, only 3 of these 4 weighting factors are actually playing the role of independent fitting parameters; 
that is, the magnitude of one of them is automatically fixed by the set of the three other ones (e.g., $w_{\mathrm{C} 2}=1 / 3-w_{\mathrm{C} 1}-w_{1}-w_{2}$ ). In summary, within this special 4-oscillator hybrid model [14], which turns out to be obviously well suited for binary materials with zinc blende structure, the normalized heat capacity shape function, $\kappa_{h}(T)(9)$, reduces to a parameterized function of just 7 independent parameters (consisting of the 4 discrete phonon temperatures $\Theta_{n}, n=1$ to 4 , in combination with the three weighting factors $w_{1}, w_{2}$, and $w_{C 1}$ ).

Furthermore we would still like to observe that, in many cases, one knows at least approximate values for the $T \rightarrow$ 0 limiting values of Debye temperatures, $\Theta_{D}(0)$ (either as results of former analyses of cryogenic heat capacity data $[13,34]$ or from independent estimations on the basis of elastic constants [35, 73-77]). This preliminary knowledge allows us, if necessary, to substitute the weight, $w_{C 1}$, of the Debye-like component, $\kappa_{C 1}\left(x_{1}(T)\right)$, by $w_{C 1} \rightarrow\left(\Theta_{1} / \Theta_{D}(0)\right)^{3}$ (according to (20)).

4.2. Additional Empirical Parameters Involved by the Isobaric Heat Capacities. It has already been pointed out above (in Section 2) that, for numerical simulations of measured isobaric heat capacity data, $C_{p}(T)$, by means of (5), it is generally necessary to envisage the inclusion of further empirical parameters (like $A_{n}, n=1,2, \ldots$, and/or $c_{1}$ ) into the fitting process. Such additional parameters have been introduced above for a quantification of the deviations, $C_{p}(T)-C_{V h}(T)>$ 0 , of measured $C_{p}(T)$ values from the theoretical (harmonic) lattice heat capacities, $C_{V h}(T) \propto \kappa_{h}(T)$ (see (4)), due to lattice anharmonicities and possible electronic contributions. The actual inclusion (or exclusion) of one or the other additional parameter depends, of course, on the concrete constellation of the whole set of experimental $C_{p}(T)$ data in consideration.

The crucial experimental basis of the present heat capacity analyses, for ZnSe and ZnTe, was provided by Kremer et al. [46] in form of unusually comprehensive and informative sets of $C_{p}^{\text {low }}(T)$ data points comprising the regions from $2 \mathrm{~K}$ up to $277 \mathrm{~K}$, for natural $\mathrm{ZnSe}$, and up to about $150 \mathrm{~K}$, for natural $\mathrm{ZnTe}$ (see the sets of $C_{p}^{\text {low }}(T)$ and $C_{p}^{\text {low }}(T) / T^{3}$ data points [46] represented by empty circles in Figures 2 and 3 , resp., and cf. Figure 8 in [46]). Yet, particularly from the inset of Figure 2 we see that the sequence of the respective $\rho_{p}(T) \equiv C_{p}(T) / T^{3}$ data points $(O)$ for $\mathrm{ZnSe}$ are indicative of some nonmonotonic (local minimum) behaviour in the liquid-helium region. This is at variance in particular to earlier $C_{p}^{\text {low }}(T)$ data $(+)$ given for the interval $1.7 \mathrm{~K}$ to $24.8 \mathrm{~K}$ by Birch (see Table 1 of [34]), which showed an exclusively monotonic behaviour of the sequence of the respective $\rho_{p}(T) \equiv C_{p}(T) / T^{3}$ data points $(+)$ in the same region.

The more or less pronounced nonmonotonic behaviours of Kremer's $\rho_{p}(T)$ data points for natural ZnSe (Figure 2) and ZnTe (Figure 3), in the vicinities of $3.7 \mathrm{~K}$ or $2.6 \mathrm{~K}$, respectively, can be ascribed to relatively weak (nevertheless not completely negligible) contributions of degenerate electronic systems to the measured $C_{p}^{\text {low }}(T)$ dependencies (in analogy to similar observations reported for semiconductor materials like Si [61], Ge [62], several III-V materials [23], and $\mathrm{FeGa}_{3}$

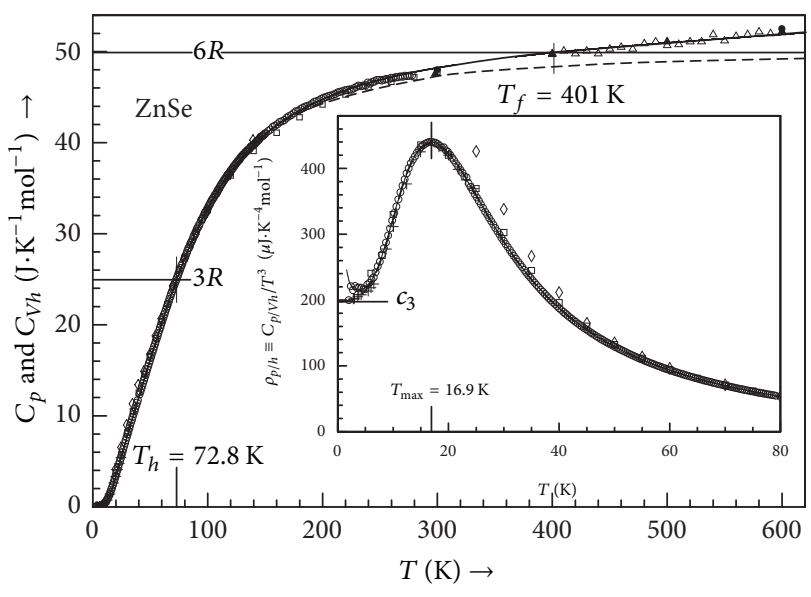

FIGURE 2: Fitting of the comprehensive set of $C_{p}^{\text {low }}(T)$ data $(O)$ provided for ZnSe by Kremer et al. [46] in combination with a series of directly measured $C_{p}^{\text {high }}(T)$ values $(\triangle)$ given for $400 \mathrm{~K} \leq T \leq$ $600 \mathrm{~K}$ by Pashinkin and Malkova (Table 1 in [78]). For comparisons with other data available for $\mathrm{ZnSe}$, we have also visualized the lowtemperature data points given by Irwin and Lacombe [33] ( $\diamond)$, Birch [34] (+), and Sirota et al. [79] (ם), including the smoothed hightemperature data points quoted by Gadzhiev et al. [80] (๑) and Pashinkin and Malkova [78] $(\mathbf{\Lambda})\left[C_{p}(T)\right.$ curve $(-$; see (5)) and $C_{V h}(T)$ curve (-----; see (4), in combination with (9) and (14) to (17)].

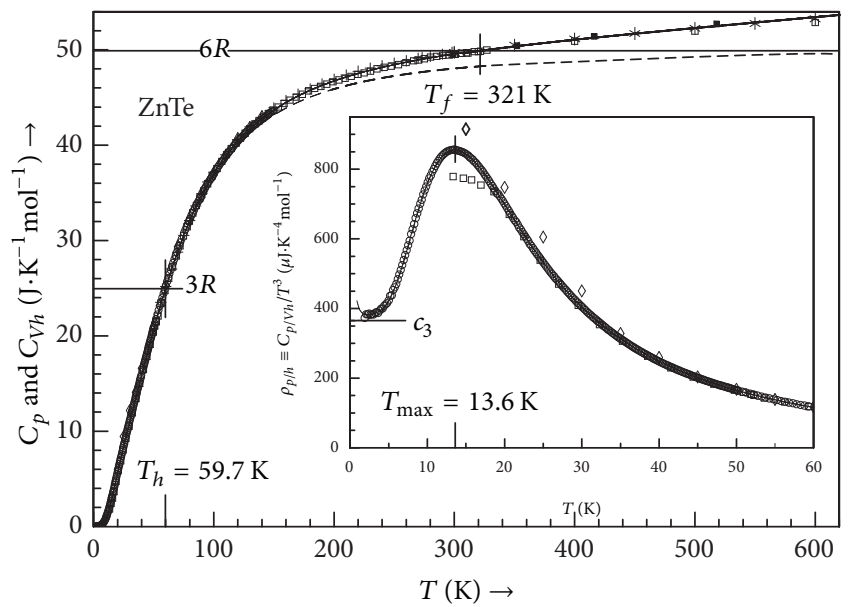

FIgURE 3: Fitting of the set of $C_{p}^{\text {low }}(T)$ data $(O)$ provided for ZnTe by Kremer et al. [46] in combination with the upper section $(102 \mathrm{~K}$ $<T<327 \mathrm{~K}$ ) of the $C_{p}^{\text {low }}(T)$ data set given by Gavrichev et al. [38] ( $\square$ ), a set of approximate $C_{p}^{\text {high }}(T)$ data points due to Gadzhiev et al. [80] (घ) (which we have redigitalized from their Figure 1), and a series of equidistant (smoothed) $C_{p}^{\text {high }}(T)$ data points $(*)$ available from the SGTE data review [81]. For comparisons with other data available for ZnTe we have also visualized the low-temperature data points given by Demidenko and Maltsev [31] (+) and Irwin and Lacombe [33] $(\diamond)$, including the smoothed high-temperature data points quoted by Gavrichev et al. [82] (口) $\left[C_{p}(T)\right.$ curve $\left(-\right.$; see (5)) and $C_{V h}(T)$ curve (- - - -; see (4), in combination with (9) and (14) to (17)]. 
TABLE 1: Sets of the adjusted model-specific parameter values, which have been obtained (for properly fixed $\Theta_{D}(0)$ values) via combined least-mean-square fittings of the presently considered sets of lowand high-temperature isobaric heat capacity data, $C_{p}(T)$, for $\mathrm{ZnSe}$ (Figure 2) and for ZnTe (Figure 3), using (5) in combination with (9) and (14) to (17). In the lower part of Table 1 we have still quoted a series of characteristic, material-specific quantities (cf. the text), the actual values of which are resulting in unambiguous way from the fitted parameter values listed previously.

\begin{tabular}{lcc}
\hline Material & ZnSe & ZnTe \\
$T$-ranges $(\mathrm{K})$ & 2 to 600 & 2 to 600 \\
\hline$\Theta_{D}^{(\text {fixed })}(0)(\mathrm{K})$ & $\mathbf{2 7 0}$ & $\mathbf{2 2 0}$ \\
$\Theta_{1(\mathrm{TA})}(\mathrm{K})$ & 78.32 & 61.93 \\
$\Theta_{2(\mathrm{TA})}(\mathrm{K})$ & 109.4 & 88.01 \\
$\Theta_{3(\mathrm{LA})}(\mathrm{K})$ & 213.0 & 167.1 \\
$\Theta_{4(\mathrm{TO} \& \mathrm{LO})}(\mathrm{K})$ & 314.4 & 263.4 \\
$w_{1}$ & 0.0632 & 0.0698 \\
$w_{2}$ & 0.2349 & 0.2331 \\
$A_{1}\left(\mathrm{~K}^{-1}\right)$ & $0.539 \times 10^{-4}$ & $0.751 \times 10^{-4}$ \\
$A_{2}\left(\mathrm{~K}^{-2}\right)$ & $0.600 \times 10^{-7}$ & $1.011 \times 10^{-7}$ \\
$c_{1}\left(\mathrm{~J} \cdot \mathrm{K}^{-2} \mathrm{~mol}{ }^{-1}\right)$ & $0.120 \times 10^{-3}$ & $0.045 \times 10^{-3}$ \\
\hline$w_{\mathrm{C} 1}=\left(\Theta_{1(\mathrm{TA})} / \Theta_{D}(0)\right)^{3}$ & 0.0244 & 0.0223 \\
$w_{\mathrm{C} 2}=1 / 3-w_{\mathrm{C} 1}-w_{1}-w_{2}$ & 0.0108 & 0.0081 \\
$c_{3}\left(\mathrm{~J} \cdot \mathrm{K}^{-4} \mathrm{~mol}^{-1}\right)$ & $0.1975 \times 10^{-3}$ & $0.3651 \times 10^{-3}$ \\
$c_{5}\left(\mathrm{~J} \cdot \mathrm{K}^{-6} \mathrm{~mol}^{-1}\right)$ & $0.667 \times 10^{-6}$ & $1.626 \times 10^{-6}$ \\
\hline$\mu_{P}^{(1)}(\mathrm{meV})$ & 19.43 & 16.05 \\
$\mu_{P}^{(2)}\left((\mathrm{meV})^{2}\right)$ & 448.2 & 308.0 \\
$\Theta_{P}(\mathrm{~K})$ & $\mathbf{2 2 5 . 5}$ & $\mathbf{1 8 5 . 8}$ \\
$\Delta_{P}$ & 0.43 & 0.45 \\
$\Theta_{D h}(\infty)(\mathrm{K})$ & 317.1 & $\mathbf{2 6 2 . 9}$ \\
\hline$C_{p}\left(T_{r}\right)(\mathrm{J} / \mathrm{K})$ & 48.19 & 49.48 \\
$S_{p}\left(T_{r}\right)(\mathrm{J} / \mathrm{K})$ & 72.12 & 82.12 \\
$H_{p}\left(T_{r}\right)-H_{p}(0)(\mathrm{kJ})$ & 10.188 & \\
\hline & & \\
\hline
\end{tabular}

[63]). Such electronic contributions are analytically represented within the present context by the respective linear (electronic) term, $C^{(E)}(T)=c_{1} T$, in (5).

The subsequent least-mean-square fitting processes are thus to be performed on the basis of (5) in combination with (9), (14) to (17), and (20). For present purposes we shall limit the sets of high-temperature $C_{p}(T)$ data, which are to be actually included into the fitting processes, to temperatures up to $600 \mathrm{~K}$ (for both materials under study). Owing to these limitations we found that couples of just the first two anharmonicity-related expansion coefficients, $A_{1}$ and $A_{2}$ (in (5)), are sufficient for good simulations of the respective hightemperature curve sections. The present least-mean-square fitting processes are thus involving simultaneous adjustments of the (more or less strongly correlated) magnitudes of altogether 10 model-specific parameters.

4.3. Combined Fittings of Low- and High-Temperature Data and Results. Concerning the case of $\mathrm{ZnSe}$ (Figure 2) we have performed a simultaneous fit of the comprehensive set of
$C_{p}^{\text {low }}(T)$ data $(O)$ given by Kremer et al. [46] in combination with a series of directly measured $C_{p}^{\text {high }}(T)$ values $(\triangle)$ given for the region $400 \mathrm{~K} \leq T \leq 600 \mathrm{~K}$ by Pashinkin and Malkova (cf. Table 1 in [78]). The resulting total set of adjusted parameters is listed in Table 1. For comparisons of the presently analyzed data with some former ones given by various authors we have still shown in Figure 2 the fine $C_{p}^{\text {low }}(T<25 \mathrm{~K})$ data points due to Birch [34] (+), the more or less strongly deviating $C_{p}^{\text {low }}(T)$ data points due to Adachi et al. $[10,33](\diamond)$ and Sirota et al. [79] ( $\square)$, two $C_{p}^{\text {high }}(T)$ data points given (for $T=300$ and $600 \mathrm{~K}$ ) by Gadzhiev et al. [80] (๑), and several smoothed $C_{p}^{\text {high }}(T)$ values estimated by Pashinkin and Malkova [78] (ム).

For the case of ZnTe (Figure 3), the present fit comprises the set of $C_{p}^{\text {low }}(T)$ data $(O)$ given by Kremer et al. [46] in combination with the upper section $(102 \mathrm{~K}<T<327 \mathrm{~K})$ of the set of $C_{p}^{\text {low }}(T)$ data points $(\square)$ given by Gavrichev et al. (in Table 1 of [38]), including a set of approximate $C_{p}^{\text {high }}(T)$ data points ( $\mathbf{a})$ due to Gadzhiev et al. (which we have redigitalized from Figure 1 shown in [80]), and a series of equidistant (smoothed) $C_{p}^{\text {high }}(T)$ data points $(*)$ available from the SGTE data review [81]. The resulting total set of adjusted parameters is listed in Table 1. For comparisons of the presently analyzed data with some former ones given by various authors we have still shown in Figure 3 the $C_{p}^{\text {low }}(T)$ data points due to Adachi et al. $[10,33](\diamond)$, Demidenko and Maltsev [31] $(+)$, and the compatible sections (up to $600 \mathrm{~K}$ ) of a series of smoothed $C_{p}^{\text {high }}(T)$ values ( $\square$ ) quoted by Gavrichev et al. [82].

The fitted (continuous) $C_{p}(T)$ and $C_{V h}(T)$ dependencies, which are implied by the material-specific sets of fitted parameter values (listed for $\mathrm{ZnSe}$ and $\mathrm{ZnTe}$ in the upper part of Table 1), are represented by solid and dashed curves, respectively, in Figures 2 and 3. In the insets of these figures we have represented the respective magnitudes of $\rho_{p / h}(T) \equiv$ $C_{p / V h}(T) / T^{3}$ ratios. The series of empty circles [46] as well as the corresponding solid curves, $\rho_{p}(T)$, show pronounced non-Debye behaviours (maxima) in the vicinities of $T_{\max }$ $=16.9 \mathrm{~K}$ (for $\mathrm{ZnSe}$ ) and $T_{\max }=13.6 \mathrm{~K}$ (for $\mathrm{ZnTe}$ ). The $c_{3}$ levels indicate the estimated $T \rightarrow 0$ limiting values, $c_{3}=$ $\rho_{h}(T \rightarrow 0)$, of the $\rho_{h}(T) \equiv C_{V h}(T) / T^{3}$ curves, which are due exclusively to the lattice contributions to the measured heat capacities; that is, $C_{V h}(T) \cong C_{p}(T)-c_{1} T$ (within the liquidhelium-hydrogen region).

In the lower part of Table 1 we have still quoted a series of characteristic, material-specific quantities, the actual values of which are resulting in unambiguous way from the fitted parameter sets. This concerns in particular the associated magnitudes (due to (19)) of the coefficients $c_{3}$ and $c_{5}$ (referring to the conventional cryogenic odd-order series expansion (6)), the first- and second-order moments of the PDOS model function, $\mu_{P}^{(1)}$ and $\mu_{P}^{(2)}$ (due to (13)), the respective average phonon temperatures $[14,21,50], \Theta_{P} \equiv \mu_{P}^{(1)} / k_{B}$, the dispersion coefficients $[14,21,50], \Delta_{P} \equiv \sqrt[2]{\mu_{P}^{(2)} /\left(\mu_{P}^{(1)}\right)^{2}-1}$, and the high-temperature $(T \rightarrow \infty)$ limiting values of the true 
Debye temperatures [14, 21, 50], $\Theta_{D h}(\infty) \equiv \sqrt[2]{(5 / 3) \mu_{P}^{(2)}} / k_{B}$ (cf. (42) below). Furthermore, with respect to the frequently considered (thermochemical) reference temperature, $T_{r} \equiv$ $298.15 \mathrm{~K}$, we have still quoted the fitted (smoothed) isobaric heat capacity values, $C_{p}\left(T_{r}\right)[14,50]$, and the respective entropy values and enthalpy differences defined by $[14,15,50]$

$$
\begin{array}{r}
S_{p}\left(T_{r}\right)=\int_{0}^{T_{r}} d T \frac{C_{p}(T)}{T}, \\
H_{p}\left(T_{r}\right)-H_{p}(0)=\int_{0}^{T_{r}} d T C_{p}(T) .
\end{array}
$$

We have given in Table 2, for a series of selected $T$ values, a list of smoothed isobaric lattice heat capacity values, $C_{p}^{(L)}(T)=C_{p}(T)-c_{1} T$, including the corresponding (effective) lattice Debye temperatures, $\Theta_{D}^{(L)}(T)$, which have been calculated on the basis of precision formulas to be presented below (in Section 5).

\section{Transformation of Heat Capacities into Debye Temperatures}

Expressive visualizations of typical non-Debye features of the temperature dependencies of heat capacities of solids are based above all on the results of point-by-point transformations of isobaric heat capacity data, $C_{p}(T)$, into the respective (effective) Debye temperature values, $\Theta_{D}(T)$. The latter are well known from Debye's classical paper [1] to be defined in implicit way $[1-4,13-15,56]$ by an integral of the form (1), where the magnitudes of the characteristic (dimensionless) variable, $x_{D}(T) \equiv \Theta_{D}(T) / T$, are representing the upper limits of integration. The original task of calculating temperature dependencies of heat capacities within the frame of Debye's theory [1] involved thus, primarily, the necessity of preparing good approximations for the dependence of Debye's modelspecific heat capacity function, $C_{D}(T) \rightarrow C_{V h}(\infty) \kappa_{D}(T)$, on $x_{D}(T)$. A detailed analytical study of the corresponding $\kappa_{D}\left(x_{D}\right)$ function (see (A.1)) is performed in Appendix A. Crucial results of this study are given, above all, in terms of approximate algebraic expressions for the respective low- and high-capacity dependencies, $\kappa_{D l}\left(x_{D}>14\right)$ and $\kappa_{D h}\left(x_{D}<\right.$ 12 ), which can be, fortunately, transformed in exact way into corresponding algebraic expressions for the respective inverse dependencies, $x_{D}^{l_{0}}(0<\kappa<0.03)$ (see (B.1)) and $x_{D}^{h_{0}}(0.05<$ $\kappa \leq 1$ ) (see (B.3)). Starting from these approximate expressions for low- and high-capacity curve sections, we succeeded to construct in Appendix B an unprecedented, integral-free analytical apparatus for approximate (more or less rough) estimations up to high-precision calculations of the complete $x_{D}(0<\kappa \leq 1)$ dependence. Let us display in explicit form the integral-free formulas which are actually to be used here for the transformations of the heat capacity data under study (for $\mathrm{ZnSe}$ and $\mathrm{ZnTe}$ ) into the respective Debye temperatures.

5.1. Asymptotic Low- and High-Temperature $\Theta_{D}(T)$ Formulas. The $x_{D} \rightarrow \infty$ asymptote of Debye's low-temperature expansion $[1,3], \kappa\left(x_{D}\right) \rightarrow\left(4 \pi^{4} / 5 x_{D}^{3}\right)($ cf. (A.4)), was already found in [13] to be of primary importance for a proper interpretation of the $\Theta_{D}(T)$ behaviour in the liquid-heliumhydrogen region. From the latter followed readily for the inverse function, $x_{D}(\kappa)$, an asymptotic dependence of the form $x_{D}(\kappa \rightarrow 0) \rightarrow x_{D}^{l_{0}}(\kappa)=\sqrt[3]{4 \pi^{4} / 5 \kappa}$ (see (B.1)). The respective limiting $(T \rightarrow 0)$ dependence of the Debye temperature, $\Theta_{D}(T) \equiv T \cdot x_{D}(T)$, was thus explicitly given by the asymptotic expression $[13,15]$

$$
\begin{aligned}
\Theta_{D}^{l_{0}}(T) & =T \cdot\left(\frac{4 \pi^{4}}{5 \kappa(T)}\right)^{1 / 3}=T \cdot\left(\frac{4 \pi^{4} C_{V h}(\infty)}{5 C_{p}(T)}\right)^{1 / 3} \\
& =T \cdot \Theta_{D}(0)\left(\frac{c_{3}}{C_{p}(T)}\right)^{1 / 3}
\end{aligned}
$$

(cf. Eq. (9) in [13]), where the last representation of the $\Theta_{D}^{l_{0}}(T)$ dependence in (25) is in accordance with the general relation $c_{3}=(4 / 5) \pi^{4} C_{V h}(\infty) /\left(\Theta_{D}(0)\right)^{3}$ (cf. Eq. (6) in [13]). However, the inherent deviations (overestimations) of approximate $\Theta_{D}^{l_{0}}(T)$ values (due to (25)) from exact $\Theta_{D}(T)$ values are found to be lower than $1 \%$ only for very low magnitudes of heat capacities, $0 \leq \kappa(T)=C_{p}(T) / C_{V h}(\infty)<0.03$, which are corresponding to $x_{D}(T)=\Theta_{D}(T) / T$ values higher than 14 (cf. Table 9 and Figure 9).

A good basis for establishing an appropriate algebraic formula for the qualitatively different behaviour at considerably smaller $x_{D}$ values is provided by the unprecedented Taylor series expansion $x_{D}^{h_{0}}\left(\kappa_{D}\right)$ (see (B.3)), which we have shown here to follow in unambiguous way for the reciprocal version, $\kappa_{D h}^{m_{R}=2}\left(x_{D}\right) \equiv\left(\kappa_{D R h}^{m_{R}=2}\left(x_{D}\right)\right)^{-1}$ (see (A.11) and (B.2)), of the $\kappa_{D R h}^{m_{R}=2}\left(x_{D}\right)$ function (see (A.10)). Accordingly, the temperature dependence of the Debye temperature, $\Theta_{D}(T) \equiv$ $T \cdot x_{D}(T)$, for moderate-to-high heat capacities, is clearly confirmed to be given by the approximate algebraic expression [15]

$$
\begin{aligned}
\Theta_{D}(T) & \cong \Theta_{D}^{h_{0}}(T) \\
& =T \cdot \sqrt[2]{-35+5 \cdot \sqrt[2]{49+56\left(\frac{C_{V h}(\infty)}{C_{p}(T)}-1\right)}} .
\end{aligned}
$$

Note that the latter formula corresponds just to Eq. (26) in [15], where it had already been taken into consideration for $\Theta_{D}(T)$ data discussions of various III-V materials. This $\Theta_{D}^{h_{0}}(T)$ formula is seen to provide a reasonable approximation (within possible deviations up to $\pm 0.7 \%$ ) to the exact $\Theta_{D}(T)$ curve throughout the range $0.05<\kappa(T)=C_{p}(T) / C_{V h}(\infty) \leq$ 1 (as shown in the upper inset of Figure 9 by the relative deviations, $\delta x_{D} / x_{D}$, of the approximate $x_{D}^{h_{0}}(\kappa)$ curve from the exact $x_{D}(\kappa)$ dependence).

5.2. Couple of High-Precision Formulas for Effective Debye Temperatures. In view of the sample character of the numerical analyses performed within the present study for the unusually fine and comprehensive $C_{p}^{\text {low }}(T)$ data provided 
TABLE 2: Smoothed values of the isobaric lattice heat capacities, $C_{p}^{(L)}(T)=C_{p}(T)-c_{1} T$, and of the corresponding (effective) Debye temperatures, $\Theta_{D}^{(L)}(T)$ (due to (27) and (28)).

\begin{tabular}{|c|c|c|c|c|}
\hline$T(\mathrm{~K})$ & $\begin{array}{c}\mathrm{ZnSe} \\
C_{p}^{(L)}\left(\mathrm{J} \cdot \mathrm{K}^{-1} \mathrm{~mol}^{-1}\right)\end{array}$ & $\begin{array}{c}\text { ZnSe } \\
\Theta_{D}^{(L)}(\mathrm{K})\end{array}$ & $\begin{array}{c}\mathrm{ZnTe} \\
C_{p}^{(L)}\left(\mathrm{J} \cdot \mathrm{K}^{-1} \mathrm{~mol}^{-1}\right)\end{array}$ & $\begin{array}{c}\mathrm{ZnTe} \\
\Theta_{D}^{(L)}(\mathrm{K})\end{array}$ \\
\hline 2 & 0.0015949 & 269.16 & 0.0029434 & 219.43 \\
\hline 4 & 0.013255 & 265.76 & 0.025043 & 214.98 \\
\hline 6 & 0.048985 & 257.84 & 0.10158 & 202.19 \\
\hline 8 & 0.13589 & 244.67 & 0.31540 & 184.80 \\
\hline 10 & 0.32260 & 229.26 & 0.75959 & 172.32 \\
\hline 12 & 0.65875 & 216.85 & 1.44623 & 166.75 \\
\hline 14 & 1.15330 & 209.86 & 2.3390 & 165.39 \\
\hline 16 & 1.78730 & 207.06 & 3.3764 & 166.53 \\
\hline 18 & 2.5396 & 206.75 & 4.4832 & 169.36 \\
\hline 20 & 3.3793 & 208.10 & 5.6015 & 173.32 \\
\hline 25 & 5.6132 & 216.48 & 8.3098 & 185.43 \\
\hline 30 & 7.7928 & 228.48 & 10.903 & 197.66 \\
\hline 35 & 9.9059 & 240.78 & 13.439 & 208.50 \\
\hline 40 & 12.002 & 251.94 & 15.931 & 217.65 \\
\hline 45 & 14.096 & 261.58 & 18.361 & 225.22 \\
\hline 50 & 16.176 & 269.74 & 20.704 & 231.40 \\
\hline 55 & 18.221 & 276.58 & 22.938 & 236.40 \\
\hline 60 & 20.210 & 282.27 & 25.045 & 240.41 \\
\hline 65 & 22.125 & 287.00 & 27.014 & 243.60 \\
\hline 70 & 23.952 & 290.91 & 28.841 & 246.12 \\
\hline 75 & 25.682 & 294.13 & 30.525 & 248.07 \\
\hline 80 & 27.309 & 296.78 & 32.071 & 249.57 \\
\hline 85 & 28.832 & 298.95 & 33.485 & 250.68 \\
\hline 90 & 30.251 & 300.72 & 34.775 & 251.47 \\
\hline 95 & 31.569 & 302.14 & 35.950 & 251.99 \\
\hline 100 & 32.790 & 303.28 & 37.021 & 252.28 \\
\hline 110 & 34.964 & 304.85 & 38.822 & 252.29 \\
\hline 120 & 36.819 & 305.68 & 40.429 & 251.69 \\
\hline 130 & 38.401 & 305.94 & 41.721 & 250.58 \\
\hline 140 & 39.755 & 305.73 & 42.807 & 249.01 \\
\hline 150 & 40.916 & 305.14 & 43.727 & 247.01 \\
\hline 160 & 41.917 & 304.19 & 44.512 & 244.57 \\
\hline 170 & 42.784 & 302.91 & 45.188 & 241.67 \\
\hline 180 & 43.540 & 301.31 & 45.774 & 238.29 \\
\hline 190 & 44.203 & 299.38 & 46.288 & 234.38 \\
\hline 200 & 44.787 & 297.12 & 46.740 & 229.88 \\
\hline 220 & 45.766 & 291.54 & 47.503 & 218.84 \\
\hline 240 & 46.522 & 284.38 & 48.122 & 204.41 \\
\hline 260 & 47.197 & 275.36 & 48.640 & 185.43 \\
\hline 280 & 47.735 & 264.13 & 49.083 & 159.77 \\
\hline 300 & 48.194 & 250.18 & 49.471 & 122.68 \\
\hline 350 & 49.100 & 197.64 & 50.283 & - \\
\hline 400 & 49.792 & 77.81 & 50.961 & - \\
\hline 450 & 50.363 & - & 51.573 & - \\
\hline 500 & 50.863 & - & 52.153 & - \\
\hline 550 & 51.321 & - & 52.722 & - \\
\hline 600 & 51.757 & - & 53.293 & - \\
\hline
\end{tabular}


by Kremer et al. [46], we have constructed in Appendix B (Section B.3) a high-precision framework for the $x_{D}(\kappa)$ dependence. This consists in a combination of a highly elaborate (12-parameter) low-capacity formula, $x_{D}^{l_{12}}(\kappa \leq$ $\kappa_{c}$ ) (see (B.8)), with a duly modified (11-parameter) highcapacity formula, $x_{D}^{h_{11}}\left(\kappa \geq \kappa_{c}\right.$ ) (see (B.9)) (where $\kappa_{c}=0.26$ represents the position of the point of crossing between the two complementary $x_{D}(\kappa)$ branches). The respective lowand high-temperature formulas for the Debye temperature, $\Theta_{D}^{l / h}(T)=T \cdot x_{D}^{l_{12} / h_{11}}(\kappa(T))$, are thus given explicitly by the couple of analytical expressions of the form

$$
\begin{aligned}
& \Theta_{D(h)}^{l}(T)=T \cdot\left[\left(\frac{4 \pi^{4}}{5 \kappa_{(h)}(T)}\right)^{1 / 3}\right. \\
& -\left(\sum_{n=2}^{10} \frac{a_{n}}{\left(\kappa_{(h)}(T)\right)^{n / 2}}\right)^{-1} \\
& \left.-\exp \left(-\frac{p}{\kappa_{(h)}(T)}\right) \sum_{n=2}^{3} g_{n}\left(\kappa_{(h)}(T)\right)^{n}\right],
\end{aligned}
$$

for the interval $0 \leq C_{p}(T) / C_{V h}(\infty)=\kappa_{(h)}(T) \leq \kappa_{c}=0.26$ (with expansion coefficients $a_{n}$, $p$, and $g_{n}$ listed in the second column of Table 8), and

$$
\begin{aligned}
\Theta_{D(h)}^{h}(T)= & T \\
& \cdot \frac{\sqrt[2]{-35+5 \cdot \sqrt[2]{49+56\left(1 / \kappa_{(h)}(T)-1\right)}}}{\left[1+\sum_{n=2}^{12} b_{n}\left(1-\kappa_{(h)}(T)\right)^{n}\right]},
\end{aligned}
$$

for the interval $0.26=\kappa_{c} \leq \kappa_{(h)}(T)=C_{p(V h)}(T) / C_{V h}(\infty) \leq 1$ (with expansion coefficients $b_{n}$ listed in the third column of Table 8). Note that we have still endowed the $\kappa(T)$ functions in (27) and (28) by an additional subscript " $h$ " (included in brackets) in order to indicate that precisely the same formulas can also be used, alternatively, for high-accuracy calculations of the "true" Debye temperatures, $\Theta_{D h}(T)$, which are visualizing the characteristic non-Debye features of the harmonic parts of lattice heat capacity curves, $C_{V h}(T)$ (see (4); Figures 4 and 5).

By means of this couple of the high-accuracy formulas (27) and (28) we have performed, first of all, the point-bypoint transformations, $C_{p}(T) \Rightarrow \Theta_{D}(T)$, for all those isobaric heat capacity data points, $C_{p}(T)$, with respect to which the concept of effective Debye temperatures is actually applicable, that is, for $C_{p}(T)<C_{V h}(\infty)=6 R$ (cf. Figures 2 and 3 ). The respective Debye temperature values, $\Theta_{D}(T)>0$, are represented for $\mathrm{ZnSe}$ (in Figure 4) and for $\mathrm{ZnTe}$ (in Figure 5) by the same symbols as the original $C_{p}(T)$ data points (shown in Figures 2 and 3, resp.).

Furthermore, we have transformed in the same way (i.e., via (27) or (28)) the continuous $C_{p}(T)$ curves (solid curves shown in Figures 2 and 3), which resulted from the preceding numerical fittings in Section 4. These continuous $\Theta_{D}(T)>0$ curves (solid curves, in Figures 4 and 5) are naturally ending

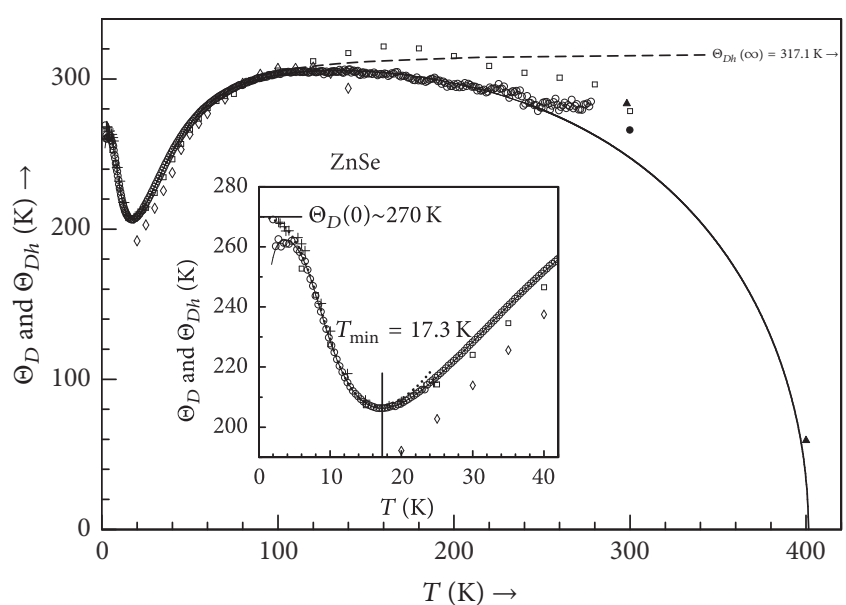

FIgURE 4: Effective Debye temperature values, $\Theta_{D}(T)$, as resulting for $\mathrm{ZnSe}$ from measured (isobaric) heat capacities, $C_{p}(T)([46](O)$, [34] (+), [10] ( $\diamond),[79](\square),[80](\bullet)$, and [78] (४)). The continuous curve for the true Debye temperature, $\Theta_{D h}(T)(-----)$, is due to (28) in combination with (9) for the isochoric (harmonic) heat capacity shape function, $\kappa_{h}(T) \equiv C_{V h}(T) / 6 R$ (according to the parameters listed in Table 1). The continuous curve for the effective Debye temperature, $\Theta_{D}(T)$ (solid curve), is due to (27) and (28) in combination with (5) and (9) for the associated isobaric (anharmonic) heat capacity shape function, $\kappa(T) \equiv C_{p}(T) / 6 R$. The dotted curve (in the inset) shows the low-temperature dependence of the true Debye temperature, $\Theta_{D h}(T)$, according to (30).

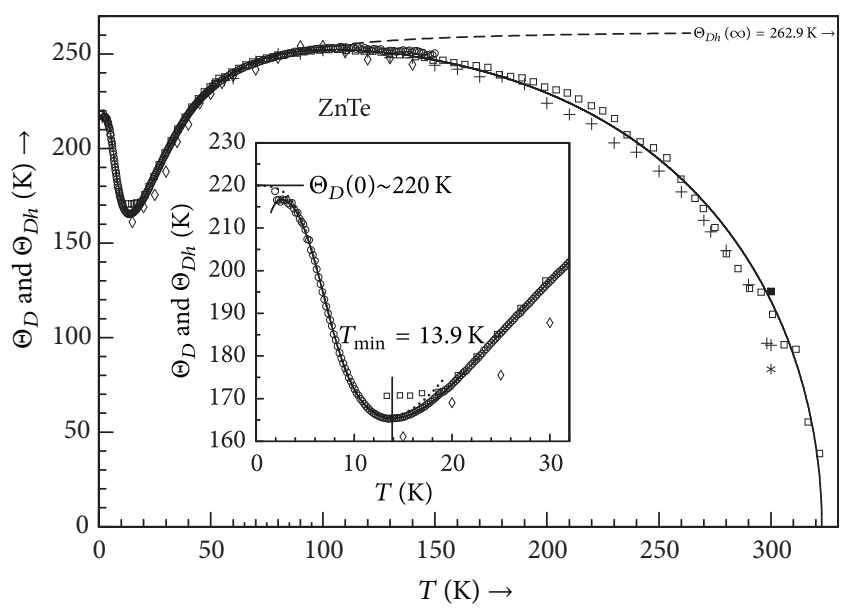

FIgURE 5: Effective Debye temperature values, $\Theta_{D}(T)$, as resulting for ZnTe from measured (isobaric) heat capacities, $C_{p}(T)([46](O)$, [38] (), [31] (+), [10] ( $\diamond),[80](\square)$, and [81] $(*))$. The continuous curve for the true Debye temperature, $\Theta_{D h}(T)(-----)$, is due to (28) in combination with (9) for the isochoric (harmonic) heat capacity shape function, $\kappa_{h}(T) \equiv C_{V h}(T) / 6 R$ (according to the parameters listed in Table 1). The continuous curve for effective Debye temperature, $\Theta_{D}(T)$ (solid curve), is due to (27) and (28) in combination with (5) and (9) for the associated isobaric (anharmonic) heat capacity shape function, $\kappa(T) \equiv C_{p}(T) / 6 R$. The dotted curve (in the inset) shows the low-temperature dependence of the true Debye temperature, $\Theta_{D h}(T)$, according to (30). 
at those temperature points, $T_{f}$, where the fitted $C_{p}(T)$ curves (in Figure 2 or 3 ) are crossing just the classical Delong-Petit heat capacity value, $C_{p}\left(T_{f}\right)=C_{V h}(\infty)=6 R$, that is, at about $401 \mathrm{~K}$ for $\mathrm{ZnSe}$ (cf. Figures 2 and 4 ) and about $323 \mathrm{~K}$ for $\mathrm{ZnTe}$ (cf. Figures 3 and 5).

5.3. Calculation of "True" (Harmonic Lattice) Debye Temperatures. Of considerable interest, particularly from theoretical points of view $[3,14,21,28,29,32,36,56,72]$, is the determination of the "true" $\left(C_{V h}(T)\right.$-related) Debye temperature curves, $\Theta_{D h}(T)$. The latter are defined with respect to the harmonic (isochoric) lattice heat capacities, $C_{V h}(T)$ (see (4)), by $[14,21]$

$$
\begin{aligned}
\frac{C_{V h}(T)}{C_{V h}(\infty)} & \equiv \kappa_{h}(T)=\frac{3}{\left(x_{D h}(T)\right)^{3}} \int_{0}^{x_{D h}(T)} d x \frac{x^{4} e^{x}}{\left(e^{x}-1\right)^{2}} \\
& \leq 1
\end{aligned}
$$

where the dimensionless variables $x_{D h}(T) \equiv \Theta_{D h}(T) / T$ are representing the respective upper boundaries of integration. Comparing (29) with (A.1) we see that the analytical connection of the upper boundaries of integration, $x_{D h}$, with the respective normalized (harmonic) heat capacity ratios, $\kappa_{h}$ (in (29)), is just the same as the preceding connection between $x_{D}$ and $\kappa$ (in (A.1)). Consequently, all the approximation formulas derived in Appendix B, for low- and high-capacity branches of the $x_{D}(\kappa)$ function (cf. Figure 9$)$, can be readily used for calculations of the $x_{D h}\left(\kappa_{h}\right)$ dependence implied by (29). This means, in particular, that (27) and (28) can be simultaneously used (as already indicated by the inclusion of the subscript " $h$ ") for high-precision calculations of the dependencies of the "true" Debye temperatures, $\Theta_{D h}(T)=$ $T \cdot x_{D h}(T)$, on the respective harmonic (isochoric) heat capacities, $C_{V h}(T)=\kappa_{h}(T) \cdot C_{V h}(\infty)$. The corresponding material-specific $\Theta_{D h}(T)$ curves are represented by dashed curves (for ZnSe and ZnTe in Figures 4 and 5, resp.).

With respect to this alternative use of (27) and (28), for calculations of $\Theta_{D h}(T)$ curves, we would still like to point out that, apart from the liquid-helium-hydrogen region (see below), appreciable differences between $\Theta_{D h}(T)$ and $\Theta_{D}(T)$ values, similarly to the differences between the underlying $C_{V h}(T)$ and $C_{p}(T)$ values (cf. Section 4 ), are encountered as a rule only in ranges of moderately low to higher temperatures, $T>T_{h}$, where the heat capacities in question are higher than about $50 \%$ of the classical Dulong-Petit limiting value, $\kappa_{h}(T)=C_{V h}(T) / C_{V h}(\infty)>0.5$. This region is automatically comprised by the range of validity of (28). Thus it is mostly not necessary to involve (27) into separate calculations of $\Theta_{D h}(T)$ curve sections.

On the other hand, significant differences between $\Theta_{D h}(T)$ and $\Theta_{D}(T)$ values may also occur at very low (liquidhelium-hydrogen) temperatures, when we are (accidentally) concerned with nonnegligible contributions, $C^{(E)}(T)=c_{1} T$, of a degenerate electronic system to the measured heat capacities, $C_{p}(T) \rightarrow C_{V h}(T)+c_{1} T$ (cf. (5)). Consequently, in order to determine the respective temperature dependence of the $\Theta_{D h}(T)$ curve within the liquid-helium-hydrogen region, we have to insert $\kappa_{h}(T)=\left(C_{p}(T)-c_{1}(T)\right) / C_{V h}(\infty)$, instead of $\kappa(T)=C_{p}(T) / C_{V h}(\infty)$, into the respective asymptotic (low- $T$ ) expression (see (25)). The monotonically decreasing sections, $d \Theta_{D h}(T) / d T<0$, of the "true" Debye temperature curves at sufficiently low temperatures, $T<T_{\text {min }}$ (see the insets of Figures 4 and 5), are thus adequately described by a corresponding asymptotic expression of the form [13]

$$
\begin{aligned}
\Theta_{D h}^{l_{0}}(T) & =T \cdot\left(\frac{4 \pi^{4}}{5 \kappa_{h}(T)}\right)^{1 / 3} \\
& =T \cdot\left(\frac{4 \pi^{4} C_{V h}(\infty)}{5 C_{V h}(T)}\right)^{1 / 3} \\
& =\Theta_{D}(0)\left(\frac{\rho_{h}(0)}{\rho_{h}(T)}\right)^{1 / 3}
\end{aligned}
$$

where $\rho_{h}(T) \equiv C_{V h}(T) / T^{3}$ and $\rho_{h}(0)=c_{3}=(4 / 5) \pi^{4} C_{V h}(\infty) /$ $\left(\Theta_{D}(0)\right)^{3}$ (see also [13] and cf. the insets of Figures 2 and 3). These asymptotic $\Theta_{D h}^{l_{0}}(T)$ dependencies are represented by dotted curve sections in the insets of Figures 4 and 5 .

\section{Discussion}

The present investigations were devoted above all to the finding of practicable solutions for longstanding computational problems which are frequently emerging within interpretations of the results of experimental heat capacity studies utilizing the somewhat troublesome concept of Debye temperatures $[1,3,4,6,10-15]$. The notorious computational complications are due to circumstance that the respective $\Theta_{D}(T)$ values are involved into the definition of the upper limits of integration, $x_{D}(T) \equiv \Theta_{D}(T) / T$, due to the integral expression for isobaric heat capacities, $C_{p}(T)$ (see (1)). Consequently, in view of the previous lack of integral-free formulas for $C_{p}(T)$ dependencies on $x_{D}(T)$ values, it was thus necessary either to calculate the individual $C_{p}(T)$ values via numerical integration procedures (using (1)) for all the individual $x_{D}(T)$ points in consideration or to determine the $C_{p}(T)=C_{V h}(\infty) \kappa(T)$ values approximately, via pointby-point interpolations, using some of the available $\kappa_{D}\left(x_{D}\right)$ tables [6, 8, 47-49].

Within our detailed study of the analytical properties of the conventional $\kappa_{D}\left(x_{D}\right)$ dependence (in Appendix A) we succeeded to derive, first of all, an unprecedented, rapidly converging high-temperature Taylor series expansion, $\kappa_{D h}^{\left(m_{R}=7\right)}\left(x_{D}\right)$ (see (A.11)). Combining the latter with Debye's conventional low-temperature expansion [1], $\kappa_{D l}^{\left(n_{D}=15\right)}\left(x_{D}\right)$ (see (A.4)), it became possible to perform high-accuracy calculations of $\kappa_{D}\left(x_{D}\right)$ values (up to at least 10 significant figures; cf. Table 7) without involving any numerical integration procedure. The subsequent adoption of respective $\kappa_{D h}\left(x_{D}\right)$ and $\kappa_{D l}\left(x_{D}\right)$ formulas to the Debyelike component, $\kappa_{C 1}\left(x_{1}(T)\right)$ (see (10)), of the long-wave TA phonon contribution to the cryogenic heat capacity, including the derivation of analogous formulas for the associated non-Debye component, $\kappa_{C 2}\left(x_{1}(T)\right)$ (see (10)), enabled us to display (in Section 3.2) an integral-free version of the multioscillator hybrid model $[14,21,50]$. This has been 
used here (in Section 4) for numerical fittings of the $C_{p}(T)$ data sets under study.

The high-accuracy results obtained in Appendix A for the $\kappa\left(x_{D}\right)$ dependence provided us, among other things, with the numerical basis for the solution of another and practically even more important mathematical problem, namely, the construction of appropriate integral-free formulas for the inverse Debye function dependence, that is, the $x_{D}(\kappa)$ function (see Figure 9 and Table 9), which describes the functional dependence of the characteristic Debye versus lattice temperature ratios, $x_{D}(T)=\Theta_{D}(T) / T$, on the ratios, $\kappa(T)=C_{p}(T) / C_{V h}(\infty)$, of given heat capacities versus Dulong-Petit classical heat capacity limit.

We have derived (in Appendix B) a series of more or less elaborate formulas for low- and high-capacity curve sections of the $x_{D}(\kappa)$ curve (solid curve in Figure 9). The simplest one of these approximate $x_{D}(\kappa)$ expressions is given in form of a junction formula, $\tilde{x}_{D}(\kappa)$ (see (B.4a)/(B.4b)), the actual use of which, however, is still connected with deviations from the exact $x_{D}(\kappa)$ dependence up to $0.6 \%$ (cf. the upper inset of Figure 9). Reductions of residual deviations to $\pm 0.13 \%$, or even to $\pm 0.0153 \%$, could be achieved (in Sections B.1 and B.2) via least-mean-square fittings of the exact $x_{D}(\kappa)$ dependence, adopting either the still relatively simple (2parameter) formula, $x_{D}^{l_{2}}\left(0<\kappa \leq \kappa_{2}\right)$ (see (B.5a) and (B.5b)), or the refined (9-parameter) formula, $x_{D}^{l_{9}}\left(0<\kappa \leq \kappa_{9}\right)$ (see (B.6)) (cf. the upper and middle insets of Figure 9, resp.). Furthermore, for the sake of high-precision calculations of Debye temperatures, we have still constructed (in Section B.3) an elaborate couple of formulas consisting of a significantly refined low-capacity (12-parameter) formula, $x_{D}^{l_{12}}(0<\kappa \leq$ $0.26)$ (see (B.8)), in combination with a similarly accurate high-capacity (11-parameter) formula, $x_{D}^{h_{11}}(0.26 \leq \kappa \leq$ 1) (see (B.9)). From the lower inset of Figure 9 we see that this couple of high-precision formulas effectuates an enormous reduction of residual deviations from the exact $x_{D}(\kappa)$ dependence by several orders of magnitude (down to $\pm 0.000016 \%)$. A set of corresponding high-precision $x_{D}(\kappa)$ values (consisting of 7 significant figures) is given, for a series of equidistant $\kappa$-points, in Table 9.

Another important aim of the present study was an exhibition of the significant progress (in comparison with [14]) in the analytical and numerical description of the heat capacity properties of $\mathrm{ZnSe}$ and $\mathrm{ZnTe}$, which we could achieve here owing to the availability of the unusually comprehensive and informative $C_{p}(T)$ data sets given by Kremer et al. [46]. A remarkable improvement for the case of $\mathrm{ZnSe}$ is due, above all, to the dense set of novel $C_{p}(T)$ data points $(O)$ for the region $2 \mathrm{~K} \leq T \leq 277 \mathrm{~K}$, which can be seen from Figure 2 to differ significantly, for $T>100 \mathrm{~K}$, from the relatively scarce (and obviously less accurate) set of former $C_{p}(T)$ data points ( $\square$ ) due to [79], which had been considered previously in [14]. The qualitative difference between the novel and the former $C_{p}(T)$ data sets for $\mathrm{ZnSe}$ is especially apparent from the visualization of the respective Debye temperature points, $\Theta_{D}(T)$, in Figure 4. As a consequence of this qualitative difference we note, above all, that the present numerical analyses (in Section 4) led to a $T \rightarrow \infty$ limiting value for the "true" (harmonic lattice) Debye temperature of $\Theta_{D h}(\infty)$ $=317.1 \mathrm{~K}$ (cf. Table 1 and Figure 4 ), which is by $1.5 \%$ lower than the former estimate (of about $322 \mathrm{~K}$; cf. [14])

Another significant progress is brought about by the unusually dense, informative sets of $C_{p}^{\text {low }}(T)$ data points available from [46] for the liquid-helium-hydrogen region $(2 \mathrm{~K} \leq T \leq 20 \mathrm{~K})$. These novel cryogenic data were of great importance particularly for the case of ZnTe, because in experimental papers published up to 2011 no $C_{p}(T)$ data at all had been given for temperatures lower than $13 \mathrm{~K}$ (and those quoted in Table 1 of [38] for the interval $13 \mathrm{~K}<T<18 \mathrm{~K}$ were obviously not sufficiently accurate; cf. the insets of Figures 3 and 5). This was the reason why it was practically impossible, prior the year 2012, to perform a direct determination of the caloric value for the $T \rightarrow 0$ limiting Debye temperature value, $\Theta_{D}(0)$ (cf. [13]).

Just with respect to the latter, however, we are now concerned, for $\mathrm{ZnTe}$, with a significant difference (of about $4.5 \%$ ) between the present estimate of $\Theta_{D}(0) \cong 220 \mathrm{~K}$ (see the inset of Figure 5) and a former estimate of $\Theta_{D}(0) \approx 230 \mathrm{~K}$ due to Kremer et al. [46]. Even a larger difference (of about 7\%) is an analogous discrepancy, for $\mathrm{ZnSe}$, between the present value of $\Theta_{D}(0) \cong 270 \mathrm{~K}$ (see the inset of Figure 4) and a former estimate of $\Theta_{D}(0) \approx 289 \mathrm{~K}$ due to Kremer et al. [46]. Let us examine in due detail the obvious causes of the discrepancies between $\Theta_{D}(0)$ values determined for $\mathrm{ZnSe}$ and $\mathrm{ZnTe}$ within the present study versus the former estimates quoted in [46].

6.1. Limiting Debye Temperatures and Electronic Heat Capacity Contributions. A provisional estimation of $\Theta_{D}(0)$ values can be made in simple way by viewing the sets of cryogenic Debye temperature points, $\Theta_{D}(T)$ (empty circles visualized in the insets of Figures 4, 5, and 6), which are exactly corresponding to the experimental $C_{p}(T) / T^{3}$ data points provided for $\mathrm{ZnSe}$ and ZnTe by Kremer et al. [46] (as shown above by empty circles in the insets of Figures 2 and 3). Concerning the lower part of the liquid-helium-hydrogen region $(2 \mathrm{~K}<T<$ $10 \mathrm{~K}$ ), we see readily that the respective $\Theta_{D}(T)$ values are throughout ranging below the following upper boundaries:

$$
\begin{array}{ll}
\Theta_{D}(T)<270 \mathrm{~K} & \text { for ZnSe, } \\
\Theta_{D}(T)<220 \mathrm{~K} & \text { for ZnTe. }
\end{array}
$$

It is remarkable that these strict limitations refer even to the lowest experimental points (which are located in the vicinity of $2 \mathrm{~K}$, in both cases).

In accordance with these mere visual estimations, it followed also from our detailed analyses (in Section 4) that the limiting $(T \rightarrow 0)$ values of Debye temperatures, $\Theta_{D}(0)$ (cf. Table 1), are nearly coinciding (within possible uncertainties of order $\pm 2 \mathrm{~K}$ ) with these upper boundaries (31); that is,

$$
\begin{array}{ll}
\Theta_{D}(0) \cong 270 K( \pm 2 \mathrm{~K}), & \text { for ZnSe, } \\
\Theta_{D}(0) \cong 220 K( \pm 2 \mathrm{~K}), & \text { for ZnTe. }
\end{array}
$$

These results are, of course, in clear contrast to the former $\Theta_{D}(0)$ estimates by Kremer et al. [46] (of about $289 \mathrm{~K}$ for $\mathrm{ZnSe}$ and $230 \mathrm{~K}$ for $\mathrm{ZnTe}$ ). Let us reveal the basic cause of these discrepancies. 
It is well known from various earlier studies of lowtemperature heat capacities of nonmetals that, within relatively small temperature regions (from 0 up to about $\left.\Theta_{D}(0) / 30\right)$, the isobaric heat capacities can as a rule be well approximated by odd-order power series expansions of the conventional form $[13,16,18,23,35,39,65]$

$$
C_{p}(T)=\sum_{n=0}^{3} c_{2 n+1} T^{2 n+1},
$$

where the $c_{1} T$ term $[4,6,13,23,60-63]$ represents a possible electronic contribution and the subsequent three oddorder terms are due to harmonic lattice contributions. Of special interest in this connection is the lowest-order lattice contribution, $c_{3} T^{3}$, which corresponds to Debye's classical $\left(T \rightarrow 0\right.$ limiting) cubic law $[1,3,6,8,21], C_{V h}(T \rightarrow 0) \propto$ $T^{3}$. The corresponding proportionality factor, $c_{3}$, is known to be connected in a simple way with the limiting Debye temperature, $\Theta_{D}(0)$, by the relation $[4,6,13,15,46,59,62]$

$$
\Theta_{D}(0)=\sqrt[3]{\frac{4 \pi^{4} C_{V h}(\infty)}{5 c_{3}}} .
$$

Consequently, within the frame of the frequently adopted $C_{p}(T) / T$ versus $T^{2}$ representation $[4,6,13,15,46,62,63,83]$

$$
\frac{C_{p}(T)}{T}=c_{1}+c_{3} T^{2}+c_{5} T^{4}+c_{7} T^{6}
$$

the coefficient $c_{3}$ corresponds just to the slopes of the $C_{p}(T) / T$ versus $T^{2}$ curves in the $T \rightarrow 0$ limit (which are represented in Figure 6 by dash-dot lines). However, these asymptotic lines used to be nearly coincident with the actual $C_{p}(T) / T$ versus $T^{2}$ curves only within very narrow intervals, from 0 to about $\Theta_{D}(0) / 100[16,18,35,83,84]$ (i.e., here up to only about $3 \mathrm{~K}$ for $\mathrm{ZnSe}$ and $2 \mathrm{~K}$ for $\mathrm{ZnTe}$ ). Consequently, in view of the complete lack of $C_{p}(T)$ data for the interval from 0 to $2 \mathrm{~K}$, there remains only the possibility of determining $c_{3}$ in combination with the other expansion coefficients (i.e., $c_{1}, c_{5}$, and $c_{7}$ ) by careful fittings via (35) of the somewhat larger $C_{p}(T) / T$ versus $T^{2}$ curve sections extending from $2 \mathrm{~K}$ up to about $\Theta_{D}(0) / 30$ (see the solid curves in Figure 6). The parameter values obtained in this way are listed in Table 3. Viewing the respective $\Theta_{D}(0)$ values (listed in the last line of Table 3) we satisfy ourselves that, within the existing uncertainty of $\pm 2 \mathrm{~K}$ (as indicated above, in (32)), they are in accordance with the results of the analyses performed in Section 4 (see Table 1 and cf. Figures 4 and 5).

On the background of the curves shown in Figure 6 one can now easily see why the rougher simulations of $C_{p}(T) / T$ data (in [46]) via a shortened series expansion of type

$$
\frac{C_{p}(T)}{T} \cong c_{3} T^{2}+c_{5} T^{4},
$$

for $T$ up to $10 \mathrm{~K}$, were a priori incapable of giving adequate results. We see that a shortened $C_{p}(T) / T$ expansion of type (36) (due to taking $c_{7}=0$, in (35)) corresponds to the dashdouble-point curves (shown in Figure 6), which begin to
TABle 3: Conventional Taylor series expansion coefficients determined by least-mean square fittings of cryogenic $C_{p}(T) / T$ data [46] for $\mathrm{ZnSe}$ and $\mathrm{ZnTe}$ (see Figure 6) via (35). The respective $\Theta_{D}(0)$ values are resulting from fitted $c_{3}$ values via (34).

\begin{tabular}{lcc}
\hline & ZnSe & ZnTe \\
\hline Intervals $(\mathrm{K})$ & 2.0 to 8.7 & 1.9 to 6.4 \\
$c_{1}\left(\mathrm{~mJ} \cdot \mathrm{K}^{-2} \mathrm{~mol}^{-1}\right)$ & 0.1339 & 0.0409 \\
$c_{3}\left(\mathrm{~m} \cdot \mathrm{K}^{-4} \mathrm{~mol}^{-1}\right)$ & 0.1937 & 0.3686 \\
$c_{5}\left(\mathrm{~mJ} \cdot \mathrm{K}^{-6} \mathrm{~mol}^{-1}\right)$ & $0.602 \times 10^{-3}$ & $0.537 \times 10^{-3}$ \\
$\left.\mathcal{c}_{7}(\mathrm{~m}) \cdot \mathrm{K}^{-8} \mathrm{~mol}^{-1}\right)$ & $0.815 \times 10^{-5}$ & $0.638 \times 10^{-4}$ \\
$\Theta_{D}(0)(\mathrm{K})$ & 271.7 & 219.3 \\
\hline
\end{tabular}

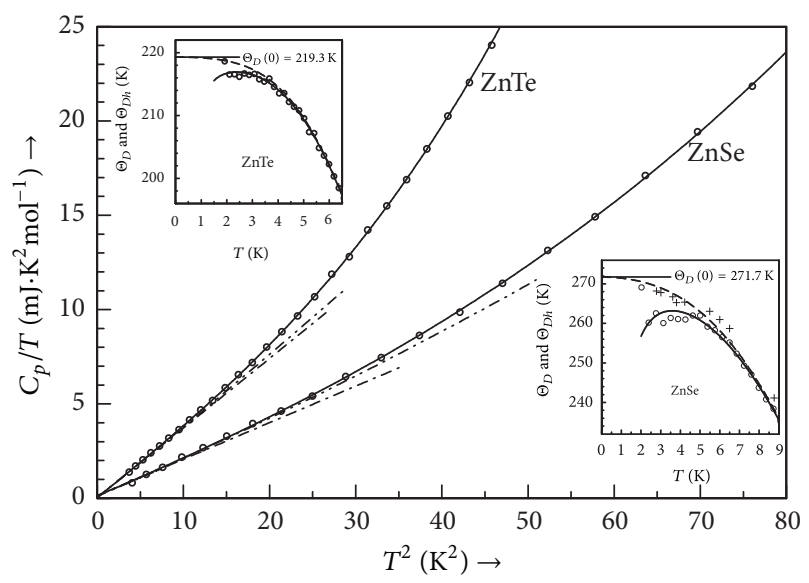

FIgure 6: Fittings via (35) of the $C_{p}(T) / T$ data points $(O)$ corresponding to the cryogenic $C_{p}(T)$ data provided by Kremer et al. [46] for $\mathrm{ZnSe}$ and $\mathrm{ZnTe}[-$, least-mean square fittings from $2 \mathrm{~K}$ to $8.7 \mathrm{~K}$ or to $6.4 \mathrm{~K}$, resp.; $\cdot \cdot-\cdot \cdot-\cdot \cdot-\cdot \cdot$, tail behaviour, for $c_{7} \rightarrow 0 ; \cdot \cdot \cdot \cdot \cdot \cdot-\cdot, T \rightarrow 0$ asymptote, for $c_{7}=0$ and $\left.c_{5}=0\right]$. The insets show the effective $\Theta_{D}(T)$ values $(O)$ following directly (via (25)) from Kremer's cryogenic $C_{p}(T)$ data [46] for ZnSe and ZnTe. For comparison we have still included the $\Theta_{D}(T)$ values $(+)$ following from former $C_{p}(T)$ data quoted for ZnSe by Birch [34], which show no indication for an appreciable electronic contribution. The continuous curves in the insets are resulting from (25), implying the cryogenic $C_{p}(T)$ series expansion (see (33)), with fitted Taylor series expansion coefficients listed in Table 3. $\left[-, \Theta_{D}(T)\right.$ curve, for $c_{1}>0 ;-----, \Theta_{D h}(T)$ curve, for $c_{1} \rightarrow 0$.]

deviate significantly from the exact (solid) curves already in the vicinity of $6 \mathrm{~K}$, for $\mathrm{ZnSe}$, or $4 \mathrm{~K}$, for $\mathrm{ZnTe}$ (i.e., at temperatures of order $\left.\Theta_{D}(0) / 50\right)$. Thus one comes unavoidably to inadequate parameter values when a shortened (twoparameter) series expansion like (36) is carelessly applied to a markedly larger interval (up to $10 \mathrm{~K}$ [46]). In accordance with this observation we have still verified that, if necessary, even by means of a shortened series expansion like (36) it is, in principle, possible to detect physically reasonable $\Theta_{D}(0)$ values (closely approaching to those quoted in Table 3), provided the fitting ranges had been strictly limited to $T<$ $6 \mathrm{~K}$, for $\mathrm{ZnSe}$, and $T<4 \mathrm{~K}$, for $\mathrm{ZnTe}$. 
In summary one can assess that the obviously erroneous $\Theta_{D}(0)$ values quoted in [46] are the inevitable consequence of a careless application of an unnecessarily shortened series expansion (of type (36)) to disproportionately extended temperature intervals (up to $10 \mathrm{~K} \mathrm{[46]).} \mathrm{At} \mathrm{the} \mathrm{same} \mathrm{time} \mathrm{we}$ have found that, even within the frame of careful applications of the full-blown low-temperature series expansion (of type (35)), one is still concerned with residual uncertainties (of about $\pm 1 \%)$ for estimated $\Theta_{D}(0)$ values. These moderate uncertainties are obviously due to certain contributions of degenerate electronic systems to the measured heat capacities, $C_{p}(T)$, which found their numerical expression in nonvanishing magnitudes for the coefficients $c_{1}$ (cf. Tables 1 and 3). Concerning the actual magnitudes of the latter we see, among other things, that the respective $c_{1}$ value for $\mathrm{ZnSe}$ is by a factor of about 3 higher than its counterpart for ZnTe. Accordingly, the electronic contribution for $\mathrm{ZnSe}$ finds its graphical representations in form of a sufficiently well discernible nonmonotonic behaviour in the vicinity of $3.7 \mathrm{~K}$, whereas the indications for a similar behaviour in the case of $\mathrm{ZnTe}$, in the vicinity of $2.6 \mathrm{~K}$, are markedly weaker (see the more or less pronounced local minimum behaviours of the $\rho_{p}(T) \equiv C_{p}(T) / T^{3}$ data points [46] in the insets of Figures 2 and 3 and the respective local maximum behaviours of the corresponding $\Theta_{D}(T)$ data points in the insets of Figures 4, 5, and 6). Our analyses have shown that the presently available cryogenic $C_{p}(T)$ data sets are not yet comprehensive enough for a more incisive quantification of the electronic contributions to the heat capacities of $\mathrm{ZnSe}$ and $\mathrm{ZnTe}$. A significant refinement would require rather detailed heat capacity data to be available especially for the interval from $1 \mathrm{~K}$ to $2 \mathrm{~K}$. The complete lack of such data is clearly the reason of the still existing uncertainties (of order $\pm 2 \mathrm{~K}$.)

We satisfy ourselves, at least, that the present estimation of $\Theta_{D}(0) \cong 270 \mathrm{~K}( \pm 2 \mathrm{~K})$, including the particular value of $\Theta_{D}(0)=271.4 \mathrm{~K}$ (Table 3 ), for $\mathrm{ZnSe}$, is in good accordance with earlier caloric values of $\Theta_{D}(0) \cong 271 \mathrm{~K}( \pm 1 \mathrm{~K}$ or $\pm 2 \mathrm{~K})$ due to Birch [34] and Collins et al. [35]. Furthermore we can make the satisfactory statement that the present estimation of $\Theta_{D}(0) \cong 220 \mathrm{~K}( \pm 2 \mathrm{~K})$, including the particular value of $\Theta_{D}(0)=219.3 \mathrm{~K}$ (Table 3 ), for $\mathrm{ZnTe}$, is in good agreement with several $\Theta_{D}^{e l}(0)$ values derived from elastic constants. This concerns in particular the $\Theta_{D}^{e l}(0)$ values of $223.2 \mathrm{~K}( \pm 5 \mathrm{~K})$ due to [35], $223.3 \mathrm{~K}$ due to [75], and $219.3 \mathrm{~K}$ due to [76]. On the other hand, one can also find in literature somewhat higher as well as markedly lower $\Theta_{D}^{e l}(0)$ values for $\mathrm{ZnTe}$, such as $225.3 \mathrm{~K}$ due to [74] and $228 \mathrm{~K}$ due to [77] or $190 \mathrm{~K}( \pm 20 \mathrm{~K})$ [38], $210 \mathrm{~K}$ due to [73], and $204.5 \mathrm{~K}$ due to [85]. Yet, in striking contrast to this multitude of earlier (mutually more or less compatible) estimates of $\Theta_{D}^{e l}(0)$ values, for ZnTe, we have still found in contemporary literature a drastically deviating quotation of $758 \mathrm{~K}$ by Guo et al. [86], which appears to be classifiable as a physically obviously absurd one.

Concerning the $\Theta_{D}(T)$ and $\Theta_{D h}(T)$ curves shown in the insets of Figure 6 we would still like to note that, by inserting the conventional odd-order power series expansions (see (33)) for the low-temperature $C_{p}(T)$ dependence into the low-capacity formula (see (25)) for effective Debye temperatures, one comes readily to a relatively simple (algebraic) $\Theta_{D}(T)$ expression of type [13]

$$
\begin{aligned}
& \Theta_{D}^{l_{0}}(T) \longrightarrow \\
& \sqrt[3]{\frac{\left(4 \pi^{4} / 5\right) \cdot C_{V h}(\infty)}{c_{1} / T^{2}+c_{3}+c_{5} T^{2}+c_{7} T^{4}}} \\
& =\frac{\Theta_{D}(0)}{\sqrt[3]{1+\left(c_{1} / T^{2}+c_{5} T^{2}+c_{7} T^{4}\right) / c_{3}}}
\end{aligned}
$$

in accordance with (34). This formula has been used here, with the parameter values quoted in Table 3 , for calculations of the low-temperature sections of the $\Theta_{D}^{l_{0}}(T)$ dependencies (solid curves in the insets of Figure 6). The associated $\Theta_{D h}^{l_{0}}(T)$ dependencies (due to (30)) for the "true" Debye temperatures, which are represented by dashed curves in the insets of Figure 6, have equally been calculated via a formula of type (37) where, merely, the electronic coefficient has been removed $\left(c_{1} \rightarrow 0\right)$. With respect to this characteristic $\Theta_{D h}^{l_{0}}(T \rightarrow 0)$ behaviour it is necessary, of course, to point out that simple algebraic formulas [13] of type (37), for $\Theta_{D}^{l_{0}}(T)$ and $\Theta_{D h}^{l_{0}}(T)$, apply only to very narrow cryogenic intervals, 0 $<T<\Theta_{D}(0) / 30$, where the respective Debye temperature curves show an exclusively convex shape (cf. the insets of Figure 6). Equivalently one can state that algebraic formula of the type (37) is not applicable to temperatures higher than $\Theta_{D}(0) / 30$ (i.e., to $T>9 \mathrm{~K}$ for $\mathrm{ZnSe}$ or $T>7 \mathrm{~K} \mathrm{ZnTe}$ ), where the (nearly coinciding) $\Theta_{D}^{l_{0}}(T)$ and $\Theta_{D h}^{l_{0}}(T)$ dependencies are showing concave shapes (cf. the insets of Figures 4 and 5).

6.2. Minima of Debye Temperature Curves and Their Interpretation. The monotonic fall of the true Debye temperatures, $\Theta_{D h}(T)$, from their limiting $(T \rightarrow 0)$ levels, $\Theta_{D}(0)$, to the respective minima, $\Theta_{D \min } \equiv \Theta_{D}\left(T_{\text {min }}\right)$, is described in good approximation by the relatively simple (algebraic) lowtemperature formula, $\Theta_{D h}^{l_{0}}(T)$ (see (30)). We see from the graphical representation of the latter by dotted curves (in the insets of Figures 4 and 5) that this $\Theta_{D h}^{l_{0}}(T)$ expression provides, indeed, close approaches to the exact $\Theta_{D h}(T)$ curves from 0 to $T_{\min } \approx \Theta_{D}(0) / 15$. With respect to these limited cryogenic regions, $0<T<\Theta_{D}(0) / 15$, one can thus describe the monotonic decrease of the ratios $\Theta_{D}(0) / \Theta_{D h}(T)$ in good approximation by the relationship $[13,14]$

$$
\frac{\Theta_{D}(0)}{\Theta_{D h}(T)} \cong \frac{\Theta_{D}(0)}{\Theta_{D h}^{l_{0}}(T)}=\sqrt[3]{\frac{\rho_{h}(T)}{\rho_{h}(0)}},
$$

in accordance with (30). The latter allows us to establish a useful approximate relationship between the minima, $\Theta_{D \min }=$ $\Theta_{D h}\left(T_{\text {min }}\right)$, of the $\Theta_{D h}(T) \rightarrow \Theta_{D}(T)$ curves, on the one hand, and the maxima, $\rho_{\max }=\rho_{h}\left(T_{\max }\right)$ of the $\rho_{h}(T) \rightarrow \rho_{p}(T)$ curves, on the other hand. Observing that the relative shifts of $T_{\min }^{(\Theta)}$ positions (indicated in the insets of Figures 4 and 5) versus the $T_{\max }^{(\rho)}$ positions (indicated in the insets of Figures 2 
and 3 ) are smaller than $2.5 \%$, in both cases (cf. also Table 4), we can hence approximate the minima of the $\Theta_{D h}(T)$ curves by $\Theta_{D \min } \cong \Theta_{D h}\left(T_{\max }^{(\rho)}\right)$. Accordingly, by specializing (38) for $T \rightarrow T_{\max }^{(\rho)} \cong T_{\min }^{(\Theta)}$, we come to an approximate relationship between the extreme values of the $\Theta_{D h}(T)$ versus $\rho_{h}(T)$ curves of the form [14]

$$
\frac{\Theta_{D}(0)}{\Theta_{D \min }} \cong \sqrt[3]{\frac{\rho_{\max }}{\rho_{h}(0)}}=\sqrt[3]{\frac{\rho_{\max }}{c_{3}}} .
$$

Comparing the actual $\Theta_{D}(0) / \Theta_{D \min }$ and $\sqrt[3]{\rho_{\max } / \rho_{h}(0)}=$ $\sqrt[3]{\rho_{\max } / c_{3}}$ values (quoted in Table 4 ) we see that approximate relation (39) is indeed well fulfilled (within deviations smaller than $0.25 \%$ ) by the empirical $\Theta_{D \min }$ and $\rho_{\max }$ values for $\mathrm{ZnSe}$ and ZnTe.

It is instructive to compare the presently obtained (empirical) $\Theta_{D \text { min }}$ values with their theoretical counterparts that had been estimated in various earlier papers. For the case of $\mathrm{ZnSe}$ we see that the previously estimated (theoretical) $\Theta_{\text {Dmin }}$ values of about $207 \mathrm{~K}$ (cf. Figure 2 in [34]) and $218 \mathrm{~K}$ (cf. Figure 14 in [36]) are in reasonable agreement with the presently determined (empirical) $\Theta_{D \min }$ value of $206.3 \mathrm{~K}$ (cf. Figure 4 and Table 4 ). In contrast to the latter, however, the theoretical $\Theta_{D}(T)$ curve presented in Figure 7 of [87] shows an exclusively monotonic behaviour for any $T>10 \mathrm{~K}$, without giving any indication of a possible local minimum behaviour in the vicinity of $T_{\min } \approx 17 \mathrm{~K}$ (cf. the inset of Figure 4 ). It is suggested by Figure 7 of [87] that the magnitude of the Debye temperature at $10 \mathrm{~K}$ should be of order $120 \mathrm{~K}$, which is about $40 \%$ lower than the actual $\Theta_{D \text { min }}$ value. A detailed numerical comparison with Figure 4 in the present paper (including Table 2) reveals, consequently, that the $\Theta_{D}(T)$ curve shown for $\mathrm{ZnSe}$ in Figure 7 of [87] is quite inadequate within a rather large cryogenic interval, from $10 \mathrm{~K}$ up to about $80 \mathrm{~K}$ (note that an analogous criticism applies also to the obviously erroneous, monotonic $\Theta_{D}(T>16 \mathrm{~K})$ curve shown for $\mathrm{ZnS}$ in Figure 7 of the same paper).

For the case of ZnTe, we see that the previously estimated $\Theta_{\text {Dmin }}$ values of about $157 \mathrm{~K}$ (cf. Figure 5 in [32]), $162 \mathrm{~K}$ (cf. Figure 1 in [35]), $159 \mathrm{~K}$ (cf. Figure 15 in [36]), and $168 \mathrm{~K}$ (cf. Figure 3 in [38]) are throughout in reasonable agreement with the presently determined (empirical) $\Theta_{D \text { min }}$ value of 165.4 K (cf. Figure 5 and Table 4).

Concerning the physical interpretation of the occurrence of the closely correlated maxima and minima of the respective $\rho_{p / h}(T)$ and $\Theta_{D / D h}(T)$ curves, we reconsider briefly the typical experimental manifestation of a heat capacity contribution, $C_{E}(T)=\left(\Theta_{E} / 2 T\right)^{2} /\left(\sinh \left(\Theta_{E} / 2 T\right)\right)^{2}$, which is due to an individual Einstein oscillator located at $\varepsilon_{E}=k_{B} \Theta_{E}$. It can easily be shown that the maximum of the respective $\rho_{E}(T) \equiv$ $C_{E}(T) / T^{3}$ curve is located at $T_{\max }^{(E)}=0.203 \Theta_{E}$ (according to Figure A1 of the supporting information to [50]). Thus, when we connect the maximum of a given $\rho_{p / h}(T)$ curve in roughest approximation with the contribution of a single Einstein oscillator, we can estimate its characteristic phonon temperature to amount to $\Theta_{E}=4.93 \cdot T_{\max }^{(E)}$. Accordingly, one can readily derive from the known $T_{\max }$ positions (of 16.9 $\mathrm{K}$ for $\mathrm{ZnSe}$ and $13.6 \mathrm{~K}$ for $\mathrm{ZnTe}$ ) values of $\Theta_{E}=83.3 \mathrm{~K}$,
TABLE 4: Pieces of evidence of the close correlation between the characteristic cryogenic non-Debye features of the $\rho_{p / h}(T) \equiv$ $C_{p / V h}(T) / T^{3}$ curves (see the insets of Figures 2 and 3 ) and the respective Debye temperature curves, $\Theta_{D}(T)$ (see Figures 4 and 5). The prominent features of the $\rho_{p / h}(T)$ curves are characterized by the $T \rightarrow 0$ levels for the lattice heat capacity, $\rho_{h}(0)=c_{3}$, the coordinates of their maxima, $T_{\max }$ and $\rho_{\max } \equiv \rho\left(T_{\max }\right)$, and the respective elevation ratios, $\rho_{\max } / \rho_{h}(0)>1$. The $\Theta_{D(h)}(T)$ curves are characterized above all by the limiting $(T \rightarrow 0)$ levels, $\Theta_{D}(0)$, the coordinates of their minima, $T_{\min }$ and $\Theta_{D \min } \equiv \Theta_{D}\left(T_{\min }\right)$, and the corresponding drop ratios, $\Theta_{D(\min )} / \Theta_{D}(0)<1$. The materialspecific ratios $\Theta_{D(\min )} / T_{\min }$ are quoted for comparisons with the general condition $\Theta_{D(\min )} / T_{\min }>14$ (cf. Section 5) of close approach to asymptotic behaviour due to (25). Furthermore, the maximum values of the effective Debye temperatures, $\Theta_{D(\max )}$, the theoretical high-temperature limiting value of the true Debye temperature, $\Theta_{D h}(\infty)$ (42), and several characteristic ratios, the approximate equality of which, for both materials, is indicating their high degree of similarity, are quoted. Particularly the large deviations from unity of the cryogenic drop ratios, $\Theta_{D(\min )} / \Theta_{D}(0) \approx 0.76$, and of the ratios for the subsequent rise to maxima, $\Theta_{D(\max )} / \Theta_{D(\min )} \approx 1.5$, illustrate the considerable strength of $T$-related variations of Debye temperatures for both materials under study.

\begin{tabular}{lcc}
\hline Material & ZnSe & ZnTe \\
\hline$\rho_{h}(0)=c_{3}\left(\mu \mathrm{J} \cdot \mathrm{K}^{-4} \mathrm{~mol}^{-1}\right)$ & 197.5 & 365.0 \\
$T_{\max }(\mathrm{K})$ & $\mathbf{1 6 . 9}$ & $\mathbf{1 3 . 6}$ \\
$\rho_{\max }\left(\mu \mathrm{J} \cdot \mathrm{K}^{-4} \mathrm{~mol}^{-1}\right)$ & 440.0 & 855.7 \\
$\rho_{\max } / \rho_{h}(0)$ & 2.228 & 2.344 \\
$\sqrt[3]{\rho_{\max } / \rho_{h}(0)}$ & 1.306 & $\mathbf{1 . 3 2 8}$ \\
\hline$\Theta_{D}(0)(\mathrm{K})$ & 270 & 220 \\
$T_{\min }(\mathrm{K})$ & 17.3 & $\mathbf{1 3 . 9}$ \\
$\Theta_{D}(0) / T_{\min }$ & 15.6 & 15.8 \\
$\Theta_{D(\min )}(\mathrm{K})$ & 206.3 & 165.4 \\
$\Theta_{D}(0) / \Theta_{D \min }$ & 1.309 & 1.330 \\
$\Theta_{D(\min )} / T_{\min }$ & 11.9 & 11.9 \\
\hline$\Theta_{D(\max )}(\mathrm{K})$ & 305.7 & 252.3 \\
$\Theta_{D(\max )} / \Theta_{D(\min )}$ & 1.482 & 1.525 \\
$\Theta_{D h}(\infty)(\mathrm{K})$ & 317 & 262 \\
$\Theta_{D}(0) / \Theta_{D h}(\infty)$ & 0.852 & 0.840 \\
$\Theta_{D(\min )} / \Theta_{D h}(\infty)$ & 0.651 & 0.631 \\
\hline
\end{tabular}

for $\mathrm{ZnSe}$, and $\Theta_{E}=70.0 \mathrm{~K}$, for $\mathrm{ZnTe}$, for the characteristic phonon temperatures of apparently dominating low-energy Einstein oscillators. Comparing these $\Theta_{E}$ values with the set of $\Theta_{n}$ values obtained by means of the detailed analyses via the four-oscillator hybrid model (in Section 4) we see that they are ranging, for both materials, within the lower parts of the intervals between the first and second Einstein oscillators,

$$
\Theta_{1(\mathrm{TA})}<\Theta_{E}<\Theta_{2(\mathrm{TA})}
$$

(cf. Table 1). Moreover, from the TA phonon dispersion relations shown for ZnSe and ZnTe in Figures 1(a) and 2(a) of [46] we infer that the characteristic TA phonon temperatures associated with the $L$ and $X$ points of the Brillouin zone boundaries, $\Theta_{\mathrm{TA}(L)}$ and $\Theta_{\mathrm{TA}(X)}$, are about $75 \mathrm{~K}$ and $93 \mathrm{~K}$, for 
ZnSe, and about $59 \mathrm{~K}$ and $77 \mathrm{~K}$, for ZnSe. Accordingly, we find that the effective Einstein oscillator temperatures, $\Theta_{E}$, are located close to the centre of gravity of the intervals between the $\Theta_{\mathrm{TA}(L)}$ and $\Theta_{\mathrm{TA}(X)}$ values; that is, $\Theta_{E} \approx\left(\Theta_{\mathrm{TA}(L)}+\Theta_{\mathrm{TA}(X)}\right) / 2$ (for both materials). These findings can be considered as a clear confirmation of various earlier assessments [14, 20, 84], according to which the occurrences of the maximum behaviours of $\rho_{p / h}(T)$ curves (like those shown in the insets of Figures 2 and 3 ) as well as the closely related minimum behaviours of the associated $\Theta_{D(h)}(T)$ curves (shown in the insets of Figures 4 and 5) are characteristic manifestations of strongly increasing contributions of short-wave TA phonons to cryogenic heat capacities.

6.3. Debye Temperature Behaviour at Higher Temperatures. In view of the analogy of the analytical connections (1) and (29), between the $x_{D}(T)=\Theta_{D}(T) / T$ ratios with $C_{p}(T)$ values, on the one hand, and the $x_{D h}(T)=\Theta_{D h}(T) / T$ ratios with $C_{V h}(T)$ values, on the other hand, it is obvious that the physical causes of the general differences, $\Theta_{D h}(T)>$ $\Theta_{D}(T)$ (see Figures 4 and 5), between "true" and effective Debye temperatures, must be sought in the mechanisms which are responsible for the inherent differences, $C_{p}(T)-$ $C_{V h}(T)>0$ (Section 2), between measured (isobaric) and theoretical (isochoric/harmonic) lattice heat capacities. For nonmagnetic materials these differences are well known to be due to

(i) possible electronic contributions, $C^{(E)}(T)=c_{1} T$ (in (5)), which are responsible for qualitatively different asymptotic behaviours, that is, $C_{p}(T) \rightarrow c_{1} T$ versus $C_{V h}(T) \rightarrow c_{3} T^{3}$, in the $T \rightarrow 0$ limit,

(ii) the cumulative effects of lattice expansion and lattice anharmonicities, which are responsible for markedly increasing differences at $T>T_{h}$ (cf. Figures 2 and 3 ).

Concerning the latter, it has been found within a series of earlier studies $[14-18,21,71,88]$ that a relatively simple way for detection of temperature dependencies of "true" Debye temperatures, $\Theta_{D h}(T)$, is offered via fittings of properly confined (i.e., exclusively concave) sections of $\Theta_{D}(T)$ versus $T^{-2}$ curves on the basis of (C.1) (see Figure 10, for the presently considered cases of $\mathrm{ZnSe}$ and $\mathrm{ZnTe}$ ). This conventional method is as a rule capable of providing good results for materials showing a sufficiently high degree of phonon dispersion like the present ones, $\Delta_{P}>1 / 3$ (see Table 1; note that this frequently encountered state of affairs is in analogy to that found, for example, for silicon and germanium [21] as well as many II-VI and III-V materials $[14,50])$. This large dispersion regime is expressively visualized by $\Theta_{D}(T)$ curves showing pronounced nonmonotonic behaviours in the vicinities of their minima (like those shown in Figures 4 and 5). The respective large degree of variability of $\Theta_{D}(T)$ values, particularly with respect to the region $T>$ $T_{\min }$, is characterized by rise ratios, $\Theta_{D \max } / \Theta_{D \min }$, that are significantly higher than unity (like $\Theta_{D \max } / \Theta_{D \min } \approx 1.5$, for $\mathrm{ZnSe}$ and ZnTe; cf. Table 4). In contrast to this, for materials with relatively small phonon dispersion, $1 / 3>\Delta_{P}>$ 1/4 (like diamond and $\mathrm{BN}$ [50], or $\mathrm{MgO}$ [17]), for which the characteristic (material-specific) $\Theta_{D \max } / \Theta_{D \min }$ ratios are known to be closely approaching to unity, this conventional method [14-18, 21, 71, 88] usually does not give good results for high-temperature behaviours, $\Theta_{D h}(T \rightarrow \infty)$, of "true" Debye temperatures (for an illustration of significant residual uncertainties involved by a tentative application of this conventional $\Theta_{D h}(T)$ estimation method to a low-dispersion material like $\mathrm{MgO}$ see [17]).

In view of the limited applicability of this conventional $\Theta_{D h}(T) \rightarrow \Theta_{D}(T)$ fitting method (cf. Appendix C) we have displayed here in due detail (in Sections 2-4) a more general, alternative method, which is based on a properly adapted multioscillator hybrid model. This model involves, above all, a physically plausible decomposition of isobaric heat capacities, $C_{p}(T)$ (see (5)), into prevailing harmonic lattice heat capacity parts, $C_{V h}(T) \propto \kappa_{h}(T)$ (see (4)), and a rapidly increasing rest term, $C_{p}(T)-C_{V h}(T) \propto\left(\kappa_{h}(T)\right)^{2} T$, which represents the cumulative effects of lattice expansion and anharmonicities. Using this analytical framework for careful least-mean-square fittings of comprehensive sets of measured $C_{p}(T)$ data, we have determined the respective parameter sets (Table 1). On the basis of the latter it was then possible to calculate in unambiguous way the corresponding harmonic lattice heat capacities, $C_{V h}(T)=C_{V h}(\infty) \kappa_{h}(T)$ (using (9), in combination with (14) to (17)). Consequently, we could here readily determine the $T$-dependencies of the "true" Debye temperatures, $\Theta_{D h}(T)$, using the high-precision formulas (27) and (28). The respective $\Theta_{D h}(T)$ dependencies are shown (by dashed curves) in Figures 4 and 5.

Comparing the limiting $\Theta_{D h}(T \rightarrow \infty)$ values listed in Table 4 (indicated by arrows in Figures 4 and 5) with their counterparts resulting from the conventional estimation procedure listed in Table 10 (indicated in Figure 10 by the intercepts of the dashed curves with the ordinate), we can discern only minor differences (of less than $0.3 \%$ ) between the limiting $\Theta_{D h}(\infty)$ values estimated via the two alternative analysis procedures. The slightly different results due to the alternative analyses procedures can thus be summarized in the form

$$
\begin{array}{ll}
\Theta_{D h}(\infty) \cong 317 \mathrm{~K}( \pm 1 \mathrm{~K}) & \text { for ZnSe } \\
\Theta_{D h}(\infty) \cong 263 \mathrm{~K}( \pm 1 \mathrm{~K}) & \text { for ZnTe. }
\end{array}
$$

The rather small size of residual uncertainties can be considered as clear indications of an obviously high degree of internal consistency and compatibility of the two qualitatively different $\Theta_{D h}(\infty)$ estimation procedures.

An additional confirmation of the apparent adequacy of these caloric $\Theta_{D h}(\infty)$ values (see (41)) can be obtained by comparison with independent theoretical estimations based on first-principle calculations of PDOS spectra. It is well known that the magnitudes of $\Theta_{D h}(\infty)$ are connected in unambiguous way with the second moments, $\mu_{P}^{(2)}$, by $[14,16$, $17,21,54,56,71,72]$

$$
\Theta_{D h}(\infty)=\frac{\sqrt[2]{(5 / 3) \mu_{P}^{(2)}}}{k_{B}} \equiv \frac{\sqrt[2]{5 / 3} \cdot \varepsilon_{P}(2)}{k_{B}},
$$


where $\varepsilon_{P}(2) \equiv \sqrt[2]{\mu_{P}^{(2)}}$ represents the associated (second order) phonon energy. We have performed within a former study [56] evaluations of moments, $\mu_{P}^{(m)}$ (see (12)), on the basis of theoretically calculated PDOS spectral functions, $g_{P}(\varepsilon)$, which had been published for a variety of group-IV, III-V, and II-VI materials in a variety of earlier papers. Among the numerical results listed in Table I of [56] one can find, among other things, corresponding $\Theta_{D h}(\infty)$ values (due to (42)) for both materials here under study, namely, $324 \mathrm{~K}$ or $319 \mathrm{~K}$ for $\mathrm{ZnSe}$ (detected from the spectra presented in Figure 8 of [36] and Figure 2 of [66], resp.), and $264 \mathrm{~K}$ or $259 \mathrm{~K}$ for ZnTe (detected from the spectra presented in Figure 9 of [36] and Figure 3 of [32], resp.). Instructive are also comparisons with graphical representations of precalculated $\Theta_{D h}(T)$ curves, from which one can detect limiting $\Theta_{D h}(T \rightarrow \infty)$ values of about $324 \mathrm{~K}$ for $\mathrm{ZnSe}$ and $259 \mathrm{~K}$ for ZnTe (as indicated by Figures 14 and 15 in [36]) and $259 \mathrm{~K}$ for $\mathrm{ZnTe}$ (as indicated by Figure 5 in [32]). In this way we satisfy ourselves that the presently obtained (caloric) $\Theta_{D h}(\infty)$ values (41) show only moderate deviations $(<2.5 \%)$ from former theoretical estimates $[32,36]$.

Concerning the high-temperature dependencies of the effective (anharmonicity-related) Debye temperatures, $\Theta_{D}(T)$, we would still like to point out that, owing to the strict limitation of their definition (see (1)) to ratios $C_{p}(T) / C_{V h}(\infty) \leq 1$, their magnitudes are automatically dropping to zero at those positions, $T_{f}$, where the individual $C_{p}(T)$ curves are crossing the classical Delong-Petit level; that is,

$$
\Theta_{D}\left(T_{f}\right)=0 \quad \text { for } C_{p}\left(T_{f}\right)=C_{V h}(\infty)=6 R .
$$

This basic property is manifested by the rapid $\Theta_{D} \rightarrow 0$ drops of the solid $\Theta_{D}(T)$ curves (in Figures 4 and 5), which are located just at those positions, $T_{f}=401 \mathrm{~K}$ (for ZnSe) or $321 \mathrm{~K}$ (for ZnTe), where the respective $C_{p}(T)$ curves are crossing the classical Dulong-Petit level, $C_{V h}(\infty)=6 R$ (as shown in Figures 2 and 3).

6.4. Characteristic PDOS-Related Phonon Energy Values. Within the frame of the presently considered four-oscillator hybrid model, the totality of quantitative information on the material-specific PDOS spectra is given (in Table 1) in terms of the adjusted sets of effective Einstein oscillator temperatures, $\Theta_{n}(n=1$ to 4$)$, and the respective weights, $w_{n}(n$ $=1$ to 4 ), including the weights $w_{C 1}$ and $w_{C 2}$ for the associated continuous low-energy curve section components (of Debye and non-Debye type, resp.). The corresponding Einstein peak energy positions, $\varepsilon_{n} \equiv k_{B} \Theta_{n}(n=1$ to 4$)$, are quoted in Table 5 . On the basis of these sets of fitted parameter values one can readily calculate a variety of characteristic dispersion-related quantities, some of which are directly comparable with their theoretical counterparts being detectible from theoretically calculated PDOS spectra presented in various former papers $[32,36,45,46,66,89]$.

Of importance for a physical understanding of the prominent role of short-wave TA phonons, which are mainly responsible for the occurrence of the characteristic nonDebye (maximum) behaviours of $C_{p}(T) / T^{3}$ curves in the liquid hydrogen region (cf. the insets of Figures 2 and 3), is also the knowledge of the centres of gravity of TA phonon sections, $\bar{\varepsilon}_{\mathrm{TA}}$. The magnitudes of the latter are given, in accordance with the respective TA phonon normalization condition (see (23)), by [14]

$$
\bar{\varepsilon}_{\mathrm{TA}}=3\left[\left(\frac{3 w_{\mathrm{C} 1}}{4}+\frac{5 w_{\mathrm{C} 2}}{6}+w_{1}\right) \varepsilon_{1}+w_{2} \varepsilon_{2}\right]
$$

(cf. Eq. (18) in [14]). Concerning the presently estimated $\bar{\varepsilon}_{T A}$ values of $8.48 \mathrm{meV}$ for ZnSe and $6.80 \mathrm{meV}$ for ZnTe (as quoted in Table 5), we can make the satisfying statement that they are in good agreement with their counterparts (of about $8.4 \mathrm{meV}$ or $8.5 \mathrm{meV}$, for $\mathrm{ZnSe}$, and $6.8 \mathrm{meV}$ or $6.9 \mathrm{meV}$, for $\mathrm{ZnTe}$ ), which we have detected from the theoretically calculated PDOS spectra presented for ZnSe and ZnTe in Figure 3 of [45] or Figure 4 of [46], respectively. Accidentally, these $\bar{\varepsilon}_{\text {TA }}$ positions are even nearly coinciding with the positions of the highest TA peaks shown within the same figures (which are readily seen to be located at about $8.5 \mathrm{meV}$ or $8.6 \mathrm{meV}$, for $\mathrm{ZnSe}$, and between $6.8 \mathrm{meV}$ and $6.9 \mathrm{meV}$, for $\mathrm{ZnTe})$.

Similarly informative are also comparisons of the average values of LA phonon energies, $\bar{\varepsilon}_{\mathrm{LA}}$, which are represented within the present formalism (due to (22)) by the third discrete oscillator, $\bar{\varepsilon}_{\mathrm{LA}}=\varepsilon_{3}$. The presently estimated $\bar{\varepsilon}_{\mathrm{LA}}$ values of $18.36 \mathrm{meV}$ for $\mathrm{ZnSe}$ and $14.40 \mathrm{meV}$ for ZnTe (cf. Table 5) are in reasonable agreement with their counterparts (of about $18.5 \mathrm{meV}$ or $19.2 \mathrm{meV}$, for ZnSe, and $14.5 \mathrm{meV}$ or $15.0 \mathrm{meV}$, for $\mathrm{ZnTe}$ ), which we have detected, again, from the theoretically calculated PDOS spectra presented in Figure 3 of [45] or Figure 4 of [46], respectively.

The average values for all acoustical (i.e., TA and LA) phonon energies, $\bar{\varepsilon}_{\text {TA\&LA }}$, are another characteristic quantity for comparisons between the results of numerical analyses of heat capacity data with theoretically calculated PDOS spectra. The analytical expression for the latter, which thus comprises the whole lower halves of PDOS spectral sections, is given within the present formalism by [14]

$$
\begin{aligned}
\bar{\varepsilon}_{L} & =\bar{\varepsilon}_{\mathrm{TA} \& \mathrm{LA}} \\
& =2\left[\left(\frac{3 w_{\mathrm{C} 1}}{4}+\frac{5 w_{\mathrm{C} 2}}{6}+w_{1}\right) \varepsilon_{1}+w_{2} \varepsilon_{2}+\frac{\varepsilon_{3}}{6}\right] \\
& =\frac{\left[2 \bar{\varepsilon}_{\mathrm{TA}}+\bar{\varepsilon}_{\mathrm{LA}}\right]}{3} .
\end{aligned}
$$

The presently estimated $\bar{\varepsilon}_{L}=\bar{\varepsilon}_{\text {TA\&LA }}$ values of $11.77 \mathrm{meV}$ for ZnSe and $9.33 \mathrm{meV}$ for ZnTe (cf. Table 5) are found again to be in reasonable agreement with their counterparts of about $11.8 \mathrm{meV}$ or $12.1 \mathrm{meV}$, for $\mathrm{ZnSe}$, and $9.4 \mathrm{meV}$ or $9.6 \mathrm{meV}$, for ZnTe, which followed immediately via (45) from the couples of $\bar{\varepsilon}_{\mathrm{TA}}$ and $\bar{\varepsilon}_{\mathrm{LA}}$ values for the average TA and LA phonon energies (which had been detected from the theoretically calculated PDOS spectra presented in Figure 3 of [45] or Figure 4 of [46], resp.). Furthermore we observe that the presently estimated (caloric) $\bar{\varepsilon}_{L}=\bar{\varepsilon}_{\text {TA\&LA }}$ values are in good agreement with our former estimations [56], which were based on theoretically calculated PDOS spectral functions published in various earlier papers, namely, $11.7 \mathrm{meV}$ due to 
TABLE 5: Phonon energy values for the 4 discrete (Einstein) oscillators, $\varepsilon_{n}=k_{B} \Theta_{n}$ ( $n=1$ to 4 , corresponding to the $\Theta_{n}$ values given in Table 1), the average phonon energies for the TA phonon sections, $\bar{\varepsilon}_{\mathrm{TA}}(44)$, and the whole acoustic phonon energy sections, $\bar{\varepsilon}_{\mathrm{TA} \& \mathrm{LA}}$ (45), including a series of equivalent (moment-related) phonon energies, $\varepsilon_{P}(m)$ (due to (46) and (47)).

\begin{tabular}{lcc}
\hline Material & ZnSe & ZnTe \\
\hline$\varepsilon_{1}=\varepsilon_{\mathrm{TA} 1}(\mathrm{meV})$ & 6.75 & 5.34 \\
$\varepsilon_{2}=\varepsilon_{\mathrm{TA} 2}(\mathrm{meV})$ & 9.43 & 7.58 \\
$\varepsilon_{3}=\bar{\varepsilon}_{\mathrm{LA}}(\mathrm{meV})$ & $\mathbf{1 8 . 3 6}$ & $\mathbf{1 4 . 4 0}$ \\
$\varepsilon_{4}=\bar{\varepsilon}_{\mathrm{TO} \& \mathrm{LO}} \equiv \bar{\varepsilon}_{U}$ & $\mathbf{2 7 . 1 0}$ & $\mathbf{2 2 . 6 9}$ \\
$(\mathrm{meV})$ & $\mathbf{8 . 4 8}$ & $\mathbf{6 . 8 0}$ \\
\hline $\bar{\varepsilon}_{\mathrm{TA}}(\mathrm{meV})$ & $\mathbf{1 1 . 7 7}$ & $\mathbf{9 . 3 3}$ \\
$\bar{\varepsilon}_{\mathrm{TA} \& \mathrm{LA}}=\bar{\varepsilon}_{L}(\mathrm{meV})$ & 10.13 & 8.18 \\
$\mathcal{\varepsilon}_{P}(-5 / 2)(\mathrm{meV})$ & 11.78 & 9.49 \\
$\varepsilon_{P}(-2)(\mathrm{meV})$ & 13.12 & 10.58 \\
$\varepsilon_{P}(-3 / 2)(\mathrm{meV})$ & 14.44 & 11.68 \\
$\varepsilon_{P}(-1)(\mathrm{meV})$ & 15.79 & 12.83 \\
$\varepsilon_{P}(-1 / 2)(\mathrm{meV})$ & 17.11 & 13.97 \\
$\varepsilon_{P}(0)(\mathrm{meV})$ & $\mathbf{1 9 . 4 3}$ & $\mathbf{1 6 . 0 1}$ \\
$\varepsilon_{P}(1)=\bar{\varepsilon}_{L \& U}(\mathrm{meV})$ & 21.17 & 17.55 \\
$\varepsilon_{P}(2)(\mathrm{meV})$ & 23.22 & 19.36 \\
$\varepsilon_{P}(4)(\mathrm{meV})$ & 24.27 & 20.29 \\
$\varepsilon_{P}(6)(\mathrm{meV})$ & 24.89 & 20.83 \\
$\varepsilon_{P}(8)(\mathrm{meV})$ & 25.30 & 21.18 \\
$\varepsilon_{P}(10)(\mathrm{meV})$ & & \\
\hline
\end{tabular}

[66, 89], or $12.5 \mathrm{meV}$ due to [36], for $\mathrm{ZnSe}$, and $9.5 \mathrm{meV}$ or $9.4 \mathrm{meV}$ due to $[32,36]$, for ZnTe.

Let us still consider the upper part of the PDOS spectra in consideration, which are consisting of one LO and two TO branches. The corresponding average optical phonon energy is given within the present formalism (due to (21)) just by the fourth discrete oscillator; that is, $\bar{\varepsilon}_{U}=\bar{\varepsilon}_{\text {TO\&LO }}=$ $\varepsilon_{4}$. The presently estimated $\bar{\varepsilon}_{U}$ values (cf. Table 5) of 27.10 $\mathrm{meV}$, for $\mathrm{ZnSe}$, and $22.69 \mathrm{meV}$, for $\mathrm{ZnTe}$, are found to be in more or less good agreement with their counterparts (of about $27.3 \mathrm{meV}$ or $25.6 \mathrm{meV}$, for $\mathrm{ZnSe}$, and $23.1 \mathrm{meV}$ or $21.7 \mathrm{meV}$, for ZnTe), which we have detected again from the theoretically calculated PDOS spectra presented in Figure 4 of [46] and Figure 3 of [45], respectively. Informative is also a comparison of present (caloric) results with our former estimations [56] of $\bar{\varepsilon}_{U}$ values on the basis of PDOS spectral functions published in various earlier papers, namely, $27.3 \mathrm{meV}, 27.1 \mathrm{meV}$, or $28.4 \mathrm{meV}$ due to $[36,66,89]$, for $\mathrm{ZnSe}$, and $22.0 \mathrm{meV}$ or $22.7 \mathrm{meV}$ due to $[32,36]$, for ZnTe. Thus we are concerned in the case of the upper (optical) PDOS spectral sections with differences between approximate $\bar{\varepsilon}_{U}$ values of different origin up to a maximum order of $5 \%$ (for both $\mathrm{ZnSe}$ and $\mathrm{ZnTe}$ ). At the same time we can see that the presently estimated (caloric) $\bar{\varepsilon}_{U}$ values (Table 5) are ranging near the middle between the highest and lowest $\bar{\varepsilon}_{U}$ estimates referring to the variety of PDOS spectra in consideration for the two materials under study. This shows that one has good chances to get reliable information on various prominent features of material-specific PDOS spectra even via dispersion-related analyses of heat capacity data.

Very informative in this respect are, above all, comparisons between moments $\mu_{P}^{(m)}$, of material-specific PDOS spectral functions $[3,4,16,18,19,21,50,52,56,69-72]$, which are generally defined by (12) (for $-3<m \neq 0$ ). Within the present formalism of the four-oscillator hybrid PDOS model function, $g_{P}(\varepsilon)$ (i.e., (7), for $n_{E}=4$ ), one obtained for the corresponding moments, $\mu_{P}^{(m)}$ (see (12)), the modelspecific expression (13) [14, 21]. Yet, for visualizations of the $m$-dependencies of such PDOS spectra moments it is convenient to represent the individual $m$ th-order moments, $\mu_{P}^{(m)}$, in terms of their equivalent $m$ th-order phonon energies $[14,16,21,50,56], \varepsilon_{P}(m) \equiv \sqrt[m]{\mu_{P}^{(m)}}$. The latter are thus given within the frame of the present four-oscillator hybrid model (in accordance with (13)) by an expression of the form $[14,21,50]$

$$
\begin{aligned}
& \varepsilon_{P}(m) \\
& =\varepsilon_{1}\left[\frac{3 w_{C 1}}{3+m}+\frac{5 w_{C 2}}{5+m}+w_{1}+\sum_{n=2}^{4} w_{n}\left(\frac{\varepsilon_{n}}{\varepsilon_{1}}\right)^{m}\right]^{1 / m}, \\
& \text { for }-3<m<0,0<m .
\end{aligned}
$$

By means of a $m \rightarrow 0$ limiting transition one obtains from (46) in particular

$$
\begin{aligned}
& \varepsilon_{P}(m \longrightarrow 0) \\
& \quad=\varepsilon_{1} \exp \left[-\frac{w_{C 1}}{3}-\frac{w_{C 2}}{5}+\sum_{n=2}^{4} w_{n} \ln \left(\frac{\varepsilon_{n}}{\varepsilon_{1}}\right)\right] .
\end{aligned}
$$

Collections of corresponding material-specific $\varepsilon_{P}(m)$ values for a series of integers and half-integers (within the interval $-3<m \leq 10$ ) are listed in Table 5 and are represented by open circles in Figure 7. In view of the fact that the whole $m$-dependence of the $\varepsilon_{P}(m)$ curve (due to (46)) divides into a couple of continuous curve sections (for $-3<m<0$ and $0<m)$, the sequence of discrete $\varepsilon_{P}(m)$ values $(O)$ are perfectly connected by the respective solid curve sections. Furthermore we have shown in Figure 7 several sets of discrete $\varepsilon_{P}(m)$ values which we have obtained via detailed numerical analyses of the theoretically calculated PDOS spectra presented for ZnSe and/or ZnTe in [32] $(\times)$, [36] $(*)$, [45] $(\diamond)$, [46] $(\triangle)$, [66] (+), and [89] ( $(\Varangle)$. The comparisons between the different $\varepsilon_{P}(m)$ values, in Figure 7, show a multitude of good agreements between the discrete $\varepsilon_{P}(m)$ values due to the PDOS spectra published by different authors, including present (caloric) results $(\mathrm{O})$. This concerns especially the region of negative orders $(m<0)$, where the actual $\varepsilon_{P}(m)$ values are predominantly due to the contributions of TA phonons. At the same time we are concerned with some markedly larger deviations in the region of positive orders $(m>0)$, where the monotonically increasing $\varepsilon_{P}(m)$ values tend to be the stronger ones governed by optical phonons the higher their orders are. The maximum difference between estimated $\varepsilon_{P}(10)$ values for $\mathrm{ZnTe}$ (due to [46] $(\triangle)$ versus [45] 




FIgURE 7: Visualization of the effective phonon energy curves, $\varepsilon_{P}(m>-3)$ (see (46); solid curves), for $\mathrm{ZnSe}$ and $\mathrm{ZnTe}$. Included are the corresponding discrete $\varepsilon_{P}(m)$ points $((O)$; cf. Table 5$)$, for integer and several negative half integer orders. For comparisons with counterparts following from former theoretically calculated PDOS spectra we have shown here also the respective series of discrete $\varepsilon_{P}(m)$ values obtained via detailed numerical analyses of the theoretically calculated PDOS spectra presented for $\mathrm{ZnSe}$ and/or $\mathrm{ZnTe}$ in [32] $(\times),[36](*),[45](\diamond),[46](\triangle),[66](+)$, and [89] (ઐ). The series of even-order $\varepsilon_{P}(m)$ values (for $m=2$ to 10 , listed in Table 10), which have been determined in Appendix $C$ via the conventional analyses procedure for Debye temperature values representing the harmonic lattice, $\Theta_{D h}(T) \cong \Theta_{D}(T)$ (cf. Figure 10), are represented by squares ( $\square)$.

$(\diamond))$ amounts to about $6 \%$, whereas, for $\mathrm{ZnSe}$, the maximum difference (due to [89] ( $\downarrow$ ) versus [45] $(\diamond)$ ) amounts even to about $13 \%$. Relatively large differences like the latter ones are obviously mainly due to significant qualitative differences between the upper (optical) sections of the theoretically calculated PDOS spectra presented by the corresponding authors (of [45] $(\diamond)$ versus [89] $(\succsim))$. At the same time we can make the satisfactory statement that the presently obtained (caloric) $\varepsilon_{P}(10)$ values (Table 5) differ from those we have derived from several other PDOS spectra in consideration for $\mathrm{ZnSe}([36](*),[46](\triangle)$, and [66] $(+))$ and for $\mathrm{ZnTe}([32](\times)$, $[36](*)$, and $[46](\triangle))$ by less than $2 \%$.
The latter findings underscore the above assessment, according to which one has as a rule good chances to get reliable information of various characteristic features of material-specific PDOS spectra by means of detailed dispersion-related analyses of heat capacity data. The rather fine quantification abilities of our elaborate analytical framework (displayed in Section 4) concern obviously also such delicate quantities like higher-order phonon energy values, $\varepsilon_{P}(m \gg 1)$, and, consequently, even the corresponding higher-order moments, $\mu_{P}^{(m)}=\left(\varepsilon_{P}(m)\right)^{m}$, of PDOS spectra.

Just with respect to the latter it might be instructive to consider still the alternative series of five even-order $\varepsilon_{P}(m)$ values (for $m=2$ to 10 ), which have been determined in Appendix $\mathrm{C}$ by means of the conventional analyses procedure [14-18, 21, 71, 88] for Debye temperatures representing the harmonic lattice properties, $\Theta_{D h}(T) \cong \Theta_{D}(T)$ (the range of which is limited to $T<96 \mathrm{~K}$, for $\mathrm{ZnSe}$, and to $T<$ $80 \mathrm{~K}$, for ZnTe; cf. Figure 10 and Table 10). The corresponding alternative $\varepsilon_{P}(m)$ values (listed in Table 10) are represented in Figure 7 by squares ( $\square$ ). Comparing the latter with their hybrid-model-related counterparts $(O)$ (listed in the last five rows of Table 5) we find excellent agreements, at least, for the lowest two orders $(m=2$ and 4$)$. Towards higher orders $(m=$ 6 to 10), however, we are concerned with growing deviations between the two alternative series of $\varepsilon_{P}(m)$ values, which are seen to increase for $\mathrm{ZnSe}$ from $2.3 \%$ to $4.4 \%$ and for $\mathrm{ZnTe}$ from $1.5 \%$ to $3.6 \%$ (cf. Figure 7 ).

Finally, let us still consider the behaviour ( $m$-dependence) of the conventional Debye-model-related energy curves, which are defined (in analogy to $[16-19,64,71]$ ) by an expression of the form

$$
\begin{aligned}
\varepsilon_{D P}(m) & \equiv\left(\frac{(3+m) \mu_{P}^{(m)}}{3}\right)^{1 / m} \\
& =\left(\frac{(3+m)}{3}\right)^{1 / m} \varepsilon_{P}(m) .
\end{aligned}
$$

in accordance with the general definition, $\varepsilon_{P}(m) \equiv\left(\mu_{P}^{(m)}\right)^{1 / m}$, of the $m$ th-order phonon energies $[14,21,50,56]$. Observing that the $m$-dependencies of the two complementary $\varepsilon_{P}(m)$ curve sections (for $-3<m<0$ and $0<m$ ) are given by (46), we come thus readily (via (48)) to the material-specific $\varepsilon_{D P}(m)$ dependencies, for $\mathrm{ZnSe}$ and $\mathrm{ZnTe}$, we have visualized by corresponding dash-dot curves in Figure 7 (note that, for the special point $m \rightarrow 0$, the particular $\varepsilon_{D P}(0)$ value follows from a corresponding limiting transition to adopt just the value $\varepsilon_{D P}(m \rightarrow 0)=e^{1 / 3} \varepsilon_{P}(0)$ (cf. $\left.[16,17]\right)$, where $\varepsilon_{P}(0)$ is given by (47)).

A prominent feature of these (conventional) Debyemodel-related energy curves, $\varepsilon_{D P}(m)$ (see (48)), is due to the well-known property $[16,17,19]$, according to which the $m \rightarrow-3$ limit of this function is automatically (exactly) equal to the $T \rightarrow 0$ limit of the cut-off energy for Debye's fictive phonon energy spectrum; that is,

$$
\varepsilon_{D P}(m \longrightarrow-3)=\left(w_{C 1}\right)^{-1 / 3} \varepsilon_{1}=k_{B} \Theta_{D}(0)=\varepsilon_{D}(0),
$$


in accordance with (20). The corresponding magnitudes of $\varepsilon_{D}(0)=23.3 \mathrm{meV}$, for $\mathrm{ZnSe}$, and $\varepsilon_{D}(0)=19.0 \mathrm{meV}$, for ZnTe, are indicated by solid triangles $(\bullet)$ in Figure 7.

Another characteristic $\varepsilon_{D P}(m)$ value is that for $m=2$, which follows readily from (48), in combination with (42), to be just equal to the $T \rightarrow \infty$ limit of the cut-off energy for Debye's fictive (harmonic) phonon energy spectrum; that is,

$$
\varepsilon_{D P}(m=2)=\sqrt[2]{\frac{5}{3}} \varepsilon_{P}(2)=k_{B} \Theta_{D h}(\infty) \equiv \varepsilon_{D h}(\infty) .
$$

The corresponding magnitudes of $\varepsilon_{D h}(\infty)=27.3 \mathrm{meV}$, for $\mathrm{ZnSe}$, and $\varepsilon_{D h}(\infty)=22.6 \mathrm{meV}$, for $\mathrm{ZnTe}$, are indicated by solid squares (ם) in Figure 7 . Accordingly, the ratios between these two limiting Debye energy (or temperature) values, $\varepsilon_{D}(0) / \varepsilon_{D h}(\infty)=\Theta_{D}(0) / \Theta_{D h}(\infty)$, amount to about 0.85 or 0.84 for $\mathrm{ZnSe}$ and $\mathrm{ZnTe}$, respectively (cf. Table 4). The significant differences (of about 15\%) between these two limiting Debye temperature values are already, per se, clear indications for a pronounced non-Debye character of both PDOS spectra in consideration.

Actually, for the hypothetical case of a properly normalized phonon energy spectrum of Debye's type, that is, for the fictive (exclusively quadratic) PDOS function $g_{D}(\varepsilon)=$ $3 \varepsilon^{2} / \varepsilon_{D c}^{3}[14,21]$ (within the interval $0 \leq \varepsilon \leq \varepsilon_{D c}$, and $g_{D}(\varepsilon)=$ 0 , for $\left.\varepsilon_{D c}<\varepsilon<\infty\right)$, the corresponding moments are readily seen to be given just by $\mu_{D}^{(m)}=3 \varepsilon_{D c}^{m} /(3+m)$ [14]. Substituting hence the actual (material-specific) moments $\mu_{P}^{(m)}$ in (48) by the moments $\mu_{D}^{(m)}$ due to Debye's fictive model, we see readily that expression (48) for $\varepsilon_{D P}(m)$ reduces just to a constant

$$
\varepsilon_{D P}(m) \longrightarrow \varepsilon_{D D}(m) \equiv\left(\frac{(3+m) \mu_{D}^{(m)}}{3}\right)^{1 / m}=\varepsilon_{D c}
$$

for any order $(-3<m<\infty)$.

Such a constancy of an $\varepsilon_{D P}(m)$ function (of type (48)), however, has never been observed in physical reality (not even in form of a relatively close approach of one or the other $\varepsilon_{D P}(m)$ curve to a horizontal line). The rather strong variations of $\varepsilon_{D P}(m)$ values shown in Figure 7, particularly the pronounced nonmonotonic (minimum) behaviours of both $\varepsilon_{D P}(m)$ curves in the vicinity of $m \approx-2$, can be looked upon as clear visual manifestations (and a global confirmation) of the obviously very pronounced non-Debye character of the PDOS spectra $[32,36,45,46,66,89]$ for both materials under study.

\section{Conclusions}

The most important progress of the present study in comparison with all earlier papers, which involved in one or the other way the somewhat delicate concept of materialspecific Debye temperatures, $\Theta_{D}(T)$, consists obviously in our elaboration (in Appendix B) of appropriate integral-free formulas for direct calculations of $\Theta_{D}(T)$ values associated with given heat capacities, $C_{p}(T)$. In view of the sample character of the present applications of this novel analytical apparatus to given isobaric and/or isochoric (harmonic) heat capacity data sets, we have performed the corresponding $C_{p / V h}(T) \Rightarrow \Theta_{D / D h}(T)$ transformations here (in Section 5) on the basis of a couple of high-accuracy formulas (27) and (28), the residual deviations from exact Debye temperature values can be seen (from the lower inset of Figure 9) to be extremely small (limited to an order of $\pm 0.000016 \%$ ). Such a high degree of accuracy, however, did not use to be actually required in practice, owing to the well-known fact that typical experimental uncertainties of measured heat capacities used to be of order $\pm 1 \%$. Couples of somewhat simpler low- and high-capacity formulas might thus also be largely adequate for future practical applications. In this respect we would like to draw attention above all to the somewhat simpler lowcapacity formulas of type (B.5a) and (B.5b) or (B.6) which, in combination with the complementary high-capacity formula (B.3), are providing already good results (within maximum deviations of orders $\pm 0.13 \%$ or $\pm 0.015 \%$, resp.; cf. Figure 9 ). In particular the latter choice appears to be quite sufficient for forthcoming numerical applications.

A crucial prerequisite for our analytical constructions and tests of appropriate analytical formulas suitable for $C_{p / V h}(T) \Rightarrow \Theta_{D / D h}(T)$ transformations (within Appendix B) consisted in a preliminary, high-accuracy determination of the general dependence of heat capacities, $C_{p / V h}(T)$, on given Debye temperatures, $\Theta_{D / D h}(T)$. An excellent solution of this conventional problem has been found in Appendix $\mathrm{A}$ in form of a couple of an unprecedented high-capacity series expansion (see (A.11)) and Debye's conventional low-capacity (low-temperature) representation [1-3] (see (A.4)). We have shown (in Appendix A) that it is rather easy to perform highaccuracy $\Theta_{D / D h}(T) \Rightarrow C_{p / V h}(T)$ transformations by means of this couple of integral-free expressions. A collection of corresponding high-accuracy sample values (consisting of 10 significant figures) for Debye's model-specific $\kappa_{D}\left(x_{D}\right)$ function (see (A.5)) is given in Table 7. In view of this extraordinarily comfortable way offered here (in Section A.1 of Appendix A) for high-accuracy calculations of heat capacities, $C_{p}(T) \equiv$ $\kappa_{D}(T) C_{V h}(\infty)$, at given magnitudes of Debye temperature versus lattice temperature ratios, $x_{D}(T)=\Theta_{D}(T) / T$, it will hence no longer be necessary either to perform point-bypoint calculations of $\kappa_{D}\left(x_{D}\right)$ values (via numerical integrations according to (A.5)) or to resort to cumbersome pointby-point interpolations between neighbouring $\kappa_{D}\left(x_{D}\right)$ values utilizing an available table with discrete $\kappa_{D}\left(x_{D}\right)$ values (like Table 7 in the present paper or one of those published in $[6,8,47-49])$.

Another significant progress with respect to the intended elaboration of a more easy-to-use analytical apparatus for heat capacity analyses within the frame of the hybrid model (Section 3) has been achieved here (in Section A.2 of Appendix A) by the derivation of couples of appropriate high- and low-capacity formulas for the normalized lowenergy TA phonon contributions of Debye and non-Debye type, $\kappa_{C 1}\left(\Theta_{1} / T\right)$ and $\kappa_{C 2}\left(\Theta_{1} / T\right)$ (see $\left.(10)\right)$, to the measured cryogenic heat capacities. Incorporating the corresponding couples of high- and low-temperature expressions (i.e., (14) to (17)) into the general formalism of the multioscillator 
hybrid model we have established a completely integralfree version of our analytical framework. Its actual use effectuated a significant acceleration of the multiparameter least-mean-square fitting processes performed (in Section 4) for fine simulations of the temperature dependencies of the comprehensive heat capacity data sets under study.

An additional important progress in quantitative respect (in comparison with our previous study [14]) could be made here owing to the availability of the unusually comprehensive and informative low-temperature heat capacity data sets given by Kremer et al. [46] for natural ZnSe and ZnTe. Of great importance just for ZnTe have been in this connection above all the unprecedented cryogenic $C_{p}(T)$ data available for the liquid-helium-hydrogen region $(2 \mathrm{~K}<T<20 \mathrm{~K})$, which gave us here for the first time the chance to perform a realistic estimation of the caloric $T \rightarrow 0$ limiting value of the Debye temperature, $\Theta_{D}(0) \cong 220 \mathrm{~K}( \pm 2 \mathrm{~K})$. Moreover, for the case of $\mathrm{ZnSe}$, the novel $C_{p}(T)$ data implied a noticeable refinement (in comparison with [14]) of the $\Theta_{D}(T)$ behaviour in the region between $100 \mathrm{~K}$ and $300 \mathrm{~K}$ (cf. Figure 4 in the present paper with Figure 12 in [14]).

Finally we would still like to point out that, in comparison with a possible use of the global (apparently universal) nonDebye formula displayed in [15], a decisive advantage of the multioscillator hybrid model used within the present study consists in the delivery of physically important byproducts in form of a multitude of adequate quantitative information (like those presented in Section 6.4) on the PDOS functions of the materials under study. At the same time one must make the reservation that the hybrid model in the specialized four-oscillator version (as displayed in Section 4) used to function quite well just for systems, the PDOS spectra of which are consisting of 3 acoustical in combination with only 3 optical phonon branches. This favourable state of affairs is encountered above all within a large variety of III-V and II-VI materials with cubic structure, including silicon and germanium. However, for systems with PDOS spectra comprising more than 3 optical branches (e.g., for binary systems with hexagonal structure, or ternary systems), it will as a rule be necessary to envisage more or less different constellations of discrete oscillators in order to come again to fine numerical simulations of corresponding heat capacity data sets.

\section{Appendix}

\section{A. Integral-Free Formulas for Debye and Non-Debye Functions}

Within Debye's original model [1], the temperature dependencies of lattice heat capacities, $C_{D}^{(L)}(T)=C_{V h}(\infty) \kappa_{D}\left(x_{D}(T)\right)$, had been assumed to be unambiguously controlled by the ratios, $x_{D}(T)=\Theta_{D} / T$, of a fixed (material-specific) Debye temperature versus lattice temperature. These dimensionless ratios are playing just the role of the upper limits of integration for the corresponding normalized heat capacity shape function of Debye's type [1]

$$
\kappa_{D}\left(x_{D}\right)=\frac{3}{x_{D}^{3}} \int_{0}^{x_{D}} d x \frac{x^{4} e^{x}}{\left(e^{x}-1\right)^{2}} \leq 1,
$$

in analogy to (1). Though this conventional model is as a rule not directly applicable to physically realistic $C_{V h}(T)$ dependencies (like those shown by the dashed curves in Figures 2 and 3), it is worth to perform here a detailed analytical study of Debye's dimensionless heat capacity shape function, $\kappa_{D}\left(x_{D}\right)$ (see (A.1)), for the following reasons:

(i) Firstly, high-accuracy results available for the $\kappa_{D}\left(x_{D}\right)$ dependence are the prerequisite for successful constructions of hitherto lacking analytical formulas for its inverse functional dependence, $x_{D}\left(\kappa_{D}\right)$ (in Appendix B).

(ii) Secondly, elaborate analytical expressions for the dependence of the $\kappa_{D}\left(\kappa_{D}\right)$ function (see (A.1)) on their upper limits of integration $\left(\right.$ ratios $x_{D}(T)=$ $\left.\Theta_{D} / T\right)$ can be directly used for representing, alternatively, the functional dependence of the characteristic low-temperature heat capacity component of Debye's type, $\kappa_{C k=1}\left(x_{1}\right)$ (see (10)), on the respective ratios $x_{1}(T)=\Theta_{1} / T($ see $(11))$.

A.1. Conventional and Alternative High-Precision Debye Function Formulas. First of all one obtains, via an integration by parts of the integral in (A.1), for the $\kappa_{D}\left(x_{D}\right)$ function the equivalent expression

$$
\kappa_{D}\left(x_{D}\right)=4 D_{3}\left(x_{D}\right)-\frac{3 x_{D}}{\left(\exp \left(x_{D}\right)-1\right)},
$$

where $D_{3}\left(x_{D}\right)$ represents the familiar Debye integral [90] for the 3-dimensional case

$$
\begin{aligned}
D_{3}\left(x_{D}\right) & \equiv \frac{3}{x_{D}^{3}} \int_{0}^{x_{D}} d x \frac{x^{3}}{e^{x}-1} \\
& =\frac{3}{x_{D}^{3}} \int_{0}^{\infty} d x \frac{x^{3}}{e^{x}-1}-\frac{3}{x_{D}^{3}} \int_{x_{D}}^{\infty} d x \frac{x^{3}}{e^{x}-1} \\
& =\frac{\pi^{4}}{5 x_{D}^{3}}-\frac{3}{x_{D}^{3}} \int_{x_{D}}^{\infty} d x \frac{x^{3}}{e^{x}-1} .
\end{aligned}
$$

Expanding then the denominator in the integrand of (A.3) into a Taylor series, $\left(e^{x}-1\right)^{-1}=\sum_{n=1}^{\infty} e^{-n x}$, and performing the integrations for the individual $x^{3} e^{-n x}$ terms from $x_{D}$ to infinity (via three subsequent integrations by parts), one obtains finally for the $\kappa_{D}\left(x_{D}\right)$ function (A.2) Debye's 
conventional low-capacity (low-temperature) series expansion $[1-3]$

$$
\begin{aligned}
& \kappa_{D l}^{n_{D}}\left(x_{D}\right)=\frac{4 \pi^{4}}{5 x_{D}^{3}}-\frac{3 x_{D}}{e^{x_{D}}-1} \\
& -12 x_{D} \sum_{n=1}^{n_{D}(\rightarrow \infty)} e^{-n x_{D}}\left(\frac{1}{n x_{D}}+\frac{3}{\left(n x_{D}\right)^{2}}+\frac{6}{\left(n x_{D}\right)^{3}}\right. \\
& \left.+\frac{6}{\left(n x_{D}\right)^{4}}\right) .
\end{aligned}
$$

We see from Figure 8, among other things, that even strongly truncated versions of this low-temperature (lowcapacity) expansion (A.4), for example, $\kappa_{D l}^{n_{D} \leq 3}\left(x_{D}\right)$, are already capable of providing rather close approaches to the exact $\kappa_{D}\left(x_{D}\right)$ curve through relatively large $x_{D}$-regions. In particular we see from the upper inset of Figure 8 that, for example, for $n_{D}=2$, the relative errors, $\delta \kappa_{D} / \kappa_{D}$, are smaller than $0.2 \%$ for any $x_{D}>3$. At the same time one can state that the accuracy of numerical calculations by means of this conventional $\kappa_{D l}^{n_{D}}\left(x_{D}\right)$ expansion (see (A.4)) increases enormously when we take into account a markedly longer series of summation terms $\left(n_{D}>10\right)$. Actually, we see in particular from the lower inset of Figure 8 that, for example, for $n_{D}=20$, the relative deviations, $\delta \kappa_{D} / \kappa_{D}$, of (A.4) from the exact $\kappa_{D}\left(x_{D}\right)$ values (A.1) are smaller than $10^{-11}$ through the interval $1.4<x_{D}<\infty$. Furthermore we see that, by extending the summation in (A.4), for example, up to $n_{D}=100$, the high-accuracy range (where $\delta \kappa_{D} / \kappa_{D}<10^{-11}$ ) comprises even a much more extended interval $\left(0.26<x_{D}<\right.$ $\infty)$. At the same time it is obvious that the expansion (A.4) is divergent in the $x_{D} \rightarrow 0$ limit. A reasonable calculation of $\kappa_{D}\left(x_{D}\right)$ values for relatively small $x_{D}$ values requires thus a qualitatively different $\kappa_{D}\left(x_{D} \rightarrow 0\right)$ expansion to be available.

Rewriting for convenience the original expression (A.1) for Debye's heat capacity shape function, $\kappa_{D}\left(x_{D}\right)$, in the equivalent form $[13,14,21]$

$$
\kappa_{D}\left(x_{D}\right)=\frac{3}{4 x_{D}^{3}} \int_{0}^{x_{D}} d z \frac{x^{4}}{(\sinh (x / 2))^{2}} \leq 1
$$

and using for the $(\sinh (x / 2))^{-2}$ function (in analogy to [16, $21,54])$ the Taylor series expansion

$$
\begin{aligned}
& \left(\sinh \left(\frac{x}{2}\right)\right)^{-2} \\
& \quad=4 x^{-2}+4 \sum_{m=1}^{\infty} \frac{(-1)^{m}(2 m-1)\left|B_{2 m}\right| x^{2 m-2}}{(2 m) !}
\end{aligned}
$$

which is convergent within the region $|x|<2 \pi$, we satisfy ourselves that the respective high-capacity (high-temperature) behaviour of the $\kappa_{D}\left(x_{D}\right)$ function (see (A.1)/(A.5)) can

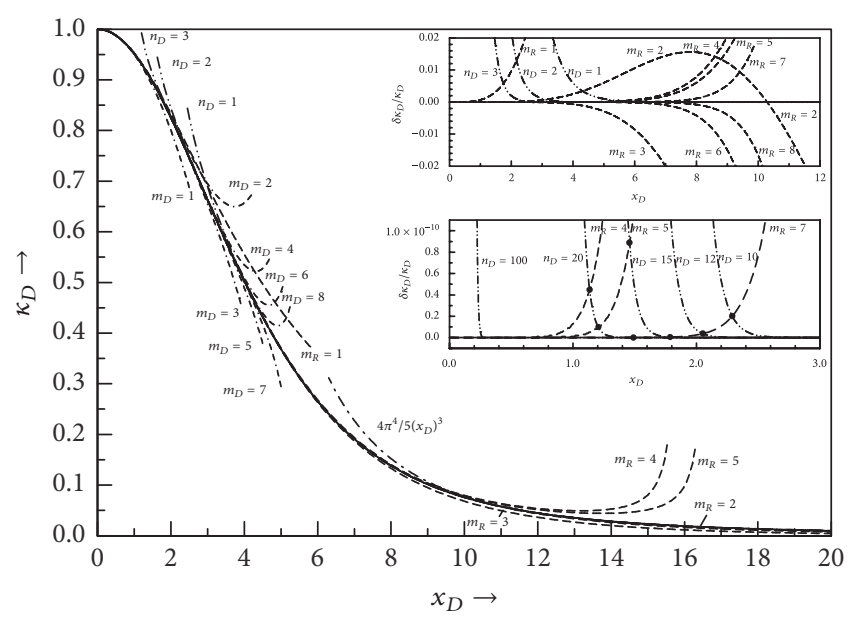

FIGURE 8: Visualization of the unusually rapid approaches of truncated versions of the presently developed (unprecedented) hightemperature $x_{D}^{2 m}$-series expansion, $\kappa_{D h}^{m_{R}}\left(x_{D}\right)(-----$; see (A.11), for $m_{R}=1$ to 8$)$, to the exact $\kappa_{D}\left(x_{D}\right)$ function (-), in comparison with the markedly slower approaches of truncated versions of Debye's conventional high-temperature $x_{D}^{2 m}$-series expansion $[1,2,16,21$, 54], $\kappa_{D h}^{m_{D}}\left(x_{D}\right)\left(--\cdot--\cdot-\right.$; see (A.7), for $m_{D}=1$ to 8$)$.

be represented (primarily) by a Taylor series expression of the conventional form $[1,2,16,21,54]$

$$
\begin{aligned}
\kappa_{D h}^{m_{D}}\left(x_{D}\right) & =1+\sum_{m=1}^{m_{D}(\rightarrow \infty)}(-1)^{m}\left|c_{2 m}^{D}\right| x_{D}^{2 m} \\
& =1+\sum_{m=1}^{m_{D}(\rightarrow \infty)} c_{2 m}^{D} x_{D}^{2 m},
\end{aligned}
$$

where the expansion coefficients for the individual evenorder $x_{D}^{2 m}$ power terms (with alternating signs), $c_{2 m}^{D}=$ $(-1)^{m}\left|c_{2 m}^{D}\right|$, are given by

$$
c_{2 m}^{D}=(-1)^{m} \frac{3(2 m-1)\left|B_{2 m}\right|}{(2 m+3) \cdot(2 m) !}, \quad m=1,2,3, \ldots .
$$

Here we have denoted by $\left|B_{2 m}\right|$ (in analogy to $[16,21,54$, 90]) the absolute values of the respective Bernoulli numbers [90] (i.e., $\left|B_{2}\right|=1 / 6,\left|B_{4}\right|=1 / 30,\left|B_{6}\right|=1 / 42,\left|B_{8}\right|=1 / 30$, $\left|B_{10}\right|=5 / 66,\left|B_{12}\right|=691 / 2730,\left|B_{14}\right|=7 / 6,\left|B_{16}\right|=$ $3617 / 510$, etc.). Accordingly, one finds the numerical values of the expansion coefficients, $c_{2 m}^{D}$, for the first eight $x_{D}^{2 m}$ power terms (in (A.7)) to be explicitly given by the following fractions:

$$
\begin{aligned}
& c_{2}^{D}=\frac{-1}{20}, \\
& c_{4}^{D}=\frac{1}{560}, \\
& c_{6}^{D}=\frac{-1}{18144},
\end{aligned}
$$




$$
\begin{aligned}
c_{8}^{D} & =\frac{1}{633600}, \\
c_{10}^{D} & =\frac{-1}{23063040}, \\
c_{12}^{D} & =\frac{691}{594397440000}, \\
c_{14}^{D} & =\frac{-1}{32572108800}, \\
c_{16}^{D} & =\frac{3617}{4505374089216000}, \ldots
\end{aligned}
$$

Note that the first 5 expansion coefficients, that is, $c_{2}^{D}$ up to $c_{10}^{D}$, had already been quoted in $[1,2]$.

However, due to the alternating signs of the $c_{2 m}^{D}$ coefficients (A.9), this conventional high-capacity (high-temperature) expansion (see (A.7)) turned out to be only a slowly converging one (as shown, in Figure 8 , by the rapidly seceding $\kappa_{D h}^{1 \leq m_{D} \leq 8}\left(x_{D}\right)$ curves following from truncated series expansions of type (A.7)). As already mentioned by Debye [1], one finds, for example, that expansions of type (A.7), which have been truncated after the $x_{D}^{6}$ or $x_{D}^{10}$ term, show deviations of less than $1 \%$ from the exact $\kappa_{D}\left(x_{D}\right)$ curve (A.1) only within rather small $x_{D}$ intervals (from 0 up to about 2 or 3 , resp.). In particular one finds that the conventional high-capacity (high-temperature) expansion (A.7) is obviously not capable of providing high-precision results for the vicinity of $x_{D} \approx 2$.

We had already found in [54] that a considerable improvement of the convergence properties of the highcapacity (high-temperature) Taylor series expansion of type $\kappa_{D h}^{m_{D}}\left(x_{D}\right)$ (see $(\mathrm{A} .7)$ ) can be achieved by a straightforward transformation of the latter, for example, into an associated (equivalent) exponential series representation. Actually, by considering (in the appendix of [54]) just the logarithm, $\kappa_{D L h}\left(x_{D}\right) \equiv \log \left(\kappa_{D h}\left(x_{D}\right)\right)$, of the original $\kappa_{D h}^{m_{D}}\left(x_{D}\right)$ function and performing a straightforward transformation of the expansion coefficients, $\left\{c_{2 m}^{D}\right\} \rightarrow\left\{c_{2 m}^{D L}\right\}$ (in analogy to the general infinite series transformation procedure sketched in Section 4.5.4 of [91]), we succeeded to construct a corresponding exponential Taylor series representation (see Eqs. (A.2) and (A.7) in [54]), which showed markedly better convergence properties than the original series expansion, $\kappa_{D h}^{1 \leq m_{D} \leq 5}\left(x_{D}\right)$, of type (A.6) (cf. Figure 6 in [54]).

Furthermore, within a more comprehensive (hitherto unpublished) analytical study performed in connection with the derivation of Eq. (3) in [15], we have found that an even faster convergence of a conveniently transformed version of the conventional high-capacity Taylor series expansion $[1,2,16,21,54], \kappa_{D h}^{m_{D}}\left(x_{D}\right)$ (see (A.7)), can be achieved when we change from the latter to a corresponding Taylor series for reciprocal $\kappa_{D h}^{-1}$ values, $\kappa_{D R h}\left(x_{D}\right) \equiv 1 / \kappa_{D h}\left(x_{D}\right)$. Performing the straightforward transformation of the expansion coefficients, $\left\{c_{2 m}^{D}\right\} \rightarrow\left\{c_{2 m}^{D R}\right\}$ [91] (which can be done in comfortable way by using, for example, a mathematical standard program like Maple), we come to an unprecedented Taylor series expansion for the reciprocal values, $\kappa_{D R h}\left(x_{D}\right)$, of the form

$$
\begin{aligned}
\kappa_{D R h}^{m_{R}}\left(x_{D}\right)= & 1+\sum_{m=1}^{m_{R}(\rightarrow \infty)} c_{2 m}^{D R} x_{D}^{2 m} \\
= & 1+\frac{x_{D}^{2}}{20}+\frac{x_{D}^{4}}{1400}+\frac{x_{D}^{6}}{648000} \\
& -\frac{73 x_{D}^{8}}{3492720000}+\cdots
\end{aligned}
$$

Note that a more extended list of the 8 lowest-order expansion coefficients, $c_{2 \leq 2 m \leq 16}^{D R}$, is given in Table 6 .

The relevant $\kappa_{D h}\left(x_{D}\right)$ function in question can be thus represented, henceforth, in terms of the reciprocal magnitudes, $\left[\kappa_{D R h}^{m_{R}}\left(x_{D}\right)\right]^{-1}$, of $\kappa_{D R h}^{m_{R}}\left(x_{D}\right)$ values (see (A.10)); that is, explicitly

$$
\begin{gathered}
\kappa_{D h}^{m_{R}}\left(x_{D}\right)=\left[1+\sum_{m=1}^{m_{R}(\rightarrow \infty)} c_{2 m}^{D R} x_{D}^{2 m}\right]^{-1}=\left[1+\frac{x_{D}^{2}}{20}\right. \\
\left.+\frac{x_{D}^{4}}{1400}+\frac{x_{D}^{6}}{648000}-\frac{73 x_{D}^{8}}{3492720000}+\cdots\right]^{-1} .
\end{gathered}
$$

We see from Figure 8 that the truncated (low-order) series expansions of the latter type, $\kappa_{D h}^{1 \leq m_{R} \leq 5}\left(x_{D}\right) \equiv\left[\kappa_{D R h}^{1 \leq m_{R} \leq 5}\left(x_{D}\right)\right]^{-1}$ (see (A.11)), are already much more rapidly converging to the exact $\kappa_{D}\left(x_{D}\right)$ dependence (solid curve) than their conventional predecessors, $\kappa_{D h}^{1 \leq m_{D} \leq 5}\left(x_{D}\right)$ (see (A.7)).

One can easily recognize the cause of the significantly improved convergence properties to be due to a qualitatively quite different constellation, of both signs and magnitudes, of expansions coefficients. Comparing in particular the magnitudes of the original expansion coefficients with the transformed ones, that is, the $\left|c_{2 m}^{D}\right|$ values (in (A.9)) with the $\left|c_{2 m}^{D R}\right|$ values (listed in Table 6), we see that the latter are decreasing much more rapidly with increasing order $(2 m=2,4,6, \ldots)$ of the individual $x_{D}^{2 m}$ power terms. In particular we see that, for example, for $m=8$, the corresponding coefficient, $c_{16}^{D R}=$ $4.952 \times 10^{-17}$ (in (A.11); cf. Table 6), is by more than 4 orders of magnitude smaller than its counterpart, $c_{16}^{D}=3617 /$ $4505374089216000=8.028 \times 10^{-13}$, due to Debye's original high-temperature Taylor series expansion (cf. (A.7)). The unusually rapid decrease of magnitudes of the transformed coefficients, $\left|c_{2 m}^{D R}\right|$, with increasing order of the $x_{D}^{2 m}$ power terms, is the main reason of the significantly improved convergence properties of the unprecedented high-capacity (high-temperature) Taylor series expansion (A.11) (as shown in Figure 8).

The truncated $\kappa_{D h}^{m_{R}=7}\left(x_{D}\right)$ version of the novel expansion (see (A.11)), which is seen (from the lower inset of Figure 8) to intersect the conventional (low-capacity) $\kappa_{D l}^{10 \leq n_{D} \leq 20}\left(x_{D}\right)$ curves somewhere within the interval $1.4<x_{D}<2.3$ (as indicated by solid circles), turned out to be especially useful for calculations of high-precision $\kappa_{D}\left(x_{D}\right)$ values. Consider in particular the point of intersection between the $\kappa_{D h}^{m_{R}=7}\left(x_{D}\right)$ 
TABLE 6: The 8 lowest-order expansion coefficients, $c_{2 \leq 2 m \leq 16}^{D R}$, implied by the unprecedented high-capacity (high-temperature) $x_{D}^{2 m}$-series expansion, $\kappa_{D h}^{m_{R}}\left(x_{D}\right)$ (A.11), for the conventional Debye function, $\kappa_{D}\left(x_{D}\right)$ (A.1). The same expansion coefficients, $c_{2 \leq 2 m \leq 16}^{D R}$, apply also the analogous $x_{1}^{2 m}$-series expansion, $\kappa_{C 1 h}^{m_{R}}\left(x_{1}\right)$ (A.15), for the Debye-like component, $\kappa_{C 1}\left(x_{1}\right)$ (A.13), of the low-energy TA phonon section of the lattice heat capacity. With respect to the latter we give here also the set of the 8 lowest-order expansion coefficients, $c_{2 \leq 2 m \leq 16}^{N R}$, which are implied by the analogous $x_{1}^{2 m}$-series expansion, $\kappa_{\mathrm{C} 2 h}^{m_{R}}\left(x_{1}\right)$ (A.23), for the associated non-Debye heat capacity component, $\kappa_{\mathrm{C} 2}\left(x_{1}\right)(\mathrm{A} .17)$.

\begin{tabular}{lll}
\hline $\mathrm{m}$ & $c_{2 m}^{D R}$ & $c_{2 m}^{N R}$ \\
\hline 1 & $\frac{1}{20}=0.05$ & $\frac{5}{84}=0.05952380952$ \\
2 & $\frac{1}{1400}=7.14285714286 \times 10^{-4}$ & $\frac{13}{10584}=1.228269085 \times 10^{-3}$ \\
3 & $\frac{1}{648000}=1.54320987654 \times 10^{-6}$ & $\frac{205}{19559232}=1.048098412 \times 10^{-5}$ \\
4 & $\frac{-73}{3492720000}=-2.09006161387 \times 10^{-8}$ & $\frac{4559}{160190110080}=2.845993424 \times 10^{-8}$ \\
5 & $\frac{1}{82555200000}=1.21131073512 \times 10^{-11}$ & $\frac{-503}{4485323082240}=-1.121435381 \times 10^{-10}$ \\
6 & $\frac{5591}{5148967824000000}=1.08584869650 \times 10^{-12}$ & $\frac{-478871}{1585247736956083200}=-3.020795986 \times 10^{-13}$ \\
7 & $\frac{-1879}{212199886080000000}=-8.85485866515 \times 10^{-15}$ & $\frac{-48299}{202404310545527029760}=-2.386261987 \times 10^{-17}$ \\
8 & $\frac{563743}{1138310922284800000000}=4.9524518213 \times 10^{-17}$ & $\frac{887853331}{16576922903367866373734400}=5.355959827 \times 10^{-17}$ \\
\hline
\end{tabular}

and $\kappa_{D l}^{n_{D}=12}\left(x_{D}\right)$ curves (being located at $\left.x_{c}=2.052\right)$, where the relative deviation, $\delta \kappa_{D} / \kappa_{D}$, of both curves from the exact $\kappa_{D}\left(x_{c}\right)$ value is $\kappa_{D h}^{m_{R}=7}\left(x_{c}\right)-\kappa_{D}\left(x_{c}\right)=\kappa_{D l}^{n_{D}=12}\left(x_{c}\right)-\kappa_{D}\left(x_{c}\right)=$ $0.37 \times 10^{-11}$. Thus, we can readily determine high-precision values for the whole $\kappa_{D}\left(x_{D}\right)$ function by using, alternatively, the functional values $\kappa_{D h}^{m_{R}=7}\left(x_{D}\right) \rightarrow \kappa_{D}\left(x_{D}\right)$, for $0 \leq x_{D} \leq$ $x_{c}$, and $\kappa_{D l}^{n_{D}=12}\left(x_{D}\right) \rightarrow \kappa_{D}\left(x_{D}\right)$, for $x_{c} \leq x_{D}<\infty$. A series of corresponding high-precision $\kappa_{D}\left(x_{D}\right)$ sample values (limited to 10 significant figures) is presented in Table 7 . The first 4 to 6 significant figures of our $\kappa_{D}\left(x_{D}\right)$ values (as well as the associated $D_{3}\left(x_{D}\right)$ values) are, of course, in complete accordance with corresponding earlier results presented in $[6,47,90]$.

By the way, the precise knowledge of the actual crossing point positions is not really necessary in view of the generally monotonic increase or decrease of the deviations of the $\kappa_{D h}^{m_{R}=7}\left(x_{D}<10\right)$ function and the $\kappa_{D l}^{n_{D}}\left(x_{D}>0\right)$ functions, respectively, from the true $\kappa_{D}\left(x_{D}\right)$ curve. It is thus quite adequate to take (with respect, e.g., to the interval $0 \leq x_{D} \leq 3$ ) simply the lower ones of the numerical values following from the two complementary $\kappa_{D}\left(x_{D}\right)$ branches; that is,

$$
\kappa_{D}\left(x_{D}\right) \longrightarrow \min \left\{\kappa_{D h}^{m_{R}=7}\left(x_{D}\right), \kappa_{D l}^{n_{D} \geq 12}\left(x_{D}\right)\right\}
$$

$$
\begin{array}{r}
\text { for } 0 \leq x_{D} \leq 3, \\
\kappa_{D}(\kappa) \longrightarrow \kappa_{D l}^{n_{D} \geq 12}\left(x_{D}\right), \text { for } 3 \leq x_{D}<\infty .
\end{array}
$$

Thus, at variance with the partly rather laborious procedures (see, e.g., [47]) involved by earlier $\kappa_{D}\left(x_{D}\right)$ calculations, we have devised here a rather comfortable way for calculations of $\kappa_{D}\left(x_{D}\right)$ values with an even considerably higher degree of accuracy (up to at least 10 significant figures), via the couple of the integral-free formulas (A.4) and (A.11). In view of this unforeseen and rather handy calculation scheme for the $\kappa_{D}\left(x_{D}\right)$ function, a use of extensive $\kappa_{D}\left(x_{D}\right)$ tables (like those presented in [6, 47-49]) appears to be completely unnecessary within the frame of possible future Debye function calculations (for 3-dimensional systems, at least).

The $m_{R}=2$ version of the $\kappa_{D h}^{m_{R}}\left(x_{D}\right)$ expansion (see (A.11)), that is, the function $\kappa_{D h}^{m_{R}=2}\left(x_{D}\right)=\left[1+x_{D}^{2} / 20+x_{D}^{4} / 1400\right]^{-1}$, just turns out to be of considerable importance within the present context for the following reasons:

(i) Firstly, as we can see from the upper inset of Figure 8, this simple function deviates by less than $2 \%$ from the exact $\kappa_{D}\left(x_{D}\right)$ dependence within an unusually large $x_{D}$-interval $\left(0 \leq x_{D}<11\right)$.

(ii) Secondly, it provides already a relatively good approximation for the inverse dependence of $x_{D}(T) \equiv$ $\Theta_{D}(T) / T$ values on heat capacity ratios, $\kappa(T) \equiv$ $C_{p}(T) / C_{V h}(\infty)>0.05$ (see (B.3) below). The inverse form of the $\kappa_{D h}^{m_{R}=2}\left(x_{D}\right)$ function plays thus an important role within the analytical apparatus (displayed in Appendix B) for constructions of reasonable $x_{D}(\kappa)$ formulas.

A.2. Integral-Free Formulas for Low-Phonon-Energy Heat Capacity Contributions. The normalized contributions, $\kappa_{C k=1}\left(x_{1}(T)\right)$ and $\kappa_{C k=2}\left(x_{1}(T)\right)$, of the low-phonon energy 
TABLE 7: High-accuracy sample values for Debye's normalized heat capacity shape function, $\kappa_{D}\left(x_{D}\right)$ (A.1), which are directly comparable with their somewhat more restricted precedents listed in $[6,47]$ (up to 6 or 8 significant figures, resp.). The individual high-accuracy $\kappa_{D}\left(x_{D}\right)$ values have been calculated here for the interval $0 \leq x_{D} \leq 1.8$, via the unprecedented high-capacity (high-temperature) Taylor series expansion, $\kappa_{D h}^{m_{R}=7}\left(x_{D}\right)$ (A.11) and, for the interval $1.8 \leq x_{D} \leq 40$, by means of Debye's conventional low-capacity (low-temperature) expansion, $\kappa_{D l}^{n_{D}=15}\left(x_{D}\right)$ (A.4) (note that a simple asymptotic expression, $\kappa_{D}\left(x_{D}\right) \rightarrow 77.92727286 / x_{D}^{3}$, applies to $x_{D}>40$ ). For comparisons with numerical results quoted in mathematical standard literature (see, e.g., Table 27.1 in [90]) we have listed here also the respective magnitudes of the conventional Debye function, $D_{3}\left(x_{D}\right)$ (for 3-dimensional systems), $D_{3}\left(x_{D}\right)=\left[\kappa_{D}\left(x_{D}\right)+3 x_{D} /\left(\exp \left(x_{D}\right)-1\right)\right] / 4(\operatorname{according}$ to (A.2) and (A.3)).

\begin{tabular}{|c|c|c|c|c|c|}
\hline$x_{D}$ & $\kappa_{D}\left(x_{D}\right)$ & $D_{3}\left(x_{D}\right)$ & $x_{D}$ & $\kappa_{D}\left(x_{D}\right)$ & $D_{3}\left(x_{D}\right)$ \\
\hline 0.0 & 1.0000000000 & 1.0000000000 & 5.0 & 0.3686348236 & 0.1175974118 \\
\hline 0.1 & 0.9995001785 & 0.9629999405 & 5.5 & 0.3132547732 & 0.09524080272 \\
\hline 0.2 & 0.9980028536 & 0.9269990483 & 6.0 & 0.2655968891 & 0.07758132473 \\
\hline 0.3 & 0.9955144242 & 0.8919951865 & 6.5 & 0.2250561623 & 0.06360434235 \\
\hline 0.4 & 0.9920454895 & 0.8579848069 & 7.0 & 0.1908564046 & 0.05250585097 \\
\hline 0.5 & 0.9876107521 & 0.8249629690 & 7.5 & 0.1621687997 & 0.04365502115 \\
\hline 0.6 & 0.9822288834 & 0.7929233677 & 8.0 & 0.1381873779 & 0.03656029567 \\
\hline 0.7 & 0.9759223556 & 0.7618583673 & 8.5 & 0.1181717647 & 0.03084031600 \\
\hline 0.8 & 0.9687172408 & 0.7317590428 & 9.0 & 0.1014670944 & 0.02619989259 \\
\hline 0.9 & 0.9606429816 & 0.7026152269 & 9.5 & 0.08750918665 & 0.02241065587 \\
\hline 1.0 & 0.9517321357 & 0.6744155641 & 10.0 & 0.07582100303 & 0.01929576569 \\
\hline 1.1 & 0.9420200996 & 0.6471475686 & 11.0 & 0.05773082402 & 0.01457049734 \\
\hline 1.2 & 0.9315448136 & 0.6207976880 & 12.0 & 0.04478011684 & 0.01125032746 \\
\hline 1.3 & 0.9203464535 & 0.5953513709 & 13.0 & 0.03534728217 & 0.0088588588804 \\
\hline 1.4 & 0.9084671129 & 0.5707931370 & 14.0 & 0.02835178383 & 0.007096677015 \\
\hline 1.5 & 0.8959504801 & 0.5471066514 & 15.0 & 0.02307128730 & 0.005771263228 \\
\hline 1.6 & 0.8828415127 & 0.5242747994 & 16.0 & 0.01901817415 & 0.004755893959 \\
\hline 1.7 & 0.8691861161 & 0.5022797638 & 17.0 & 0.01585873694 & 0.003965212076 \\
\hline 1.8 & 0.8550308264 & 0.4811031024 & 18.0 & 0.01336097602 & 0.003340449610 \\
\hline 1.9 & 0.8404225040 & 0.4607258256 & 19.0 & 0.01136091855 & 0.002840309476 \\
\hline 2.0 & 0.8254080384 & 0.4411284737 & 20.0 & 0.009740756601 & 0.002435220067 \\
\hline 2.2 & 0.7943467192 & 0.4041938106 & 21.0 & 0.008414505229 & 0.002103638250 \\
\hline 2.4 & 0.7622123728 & 0.3701368830 & 22.0 & 0.007318466926 & 0.001829621334 \\
\hline 2.6 & 0.7293549760 & 0.3387926100 & 23.0 & 0.006404797367 & 0.001601201112 \\
\hline 2.8 & 0.6961028658 & 0.3099951582 & 24.0 & 0.005637096943 & 0.001409274915 \\
\hline 3.0 & 0.6627580442 & 0.2835798281 & 25.0 & 0.004987344231 & 0.001246836318 \\
\hline 3.2 & 0.6295928776 & 0.2593847028 & 26.0 & 0.004433731487 & 0.001108432971 \\
\hline 3.4 & 0.5968481116 & 0.2372520497 & 28.0 & 0.003549893920 & 0.0008874734947 \\
\hline 3.6 & 0.5647320637 & 0.2170294741 & 30.0 & 0.002886195280 & 0.0007215488222 \\
\hline 3.8 & 0.5334208309 & 0.1985708314 & 32.0 & 0.002378151635 & 0.0005945379091 \\
\hline 4.0 & 0.5030593309 & 0.1817369138 & 34.0 & 0.001982680461 & 0.0004956701152 \\
\hline 4.2 & 0.4737629932 & 0.1663959309 & 36.0 & 0.001670251904 & 0.0004175629760 \\
\hline 4.4 & 0.4456199253 & 0.1524238050 & 38.0 & 0.001420164616 & 0.0003550411541 \\
\hline 4.6 & 0.4186933946 & 0.1397043074 & 40.0 & 0.001217613638 & 0.0003044034095 \\
\hline 4.8 & 0.3930244869 & 0.1281290579 & & & \\
\hline
\end{tabular}

terms, $\propto \varepsilon^{2}$ and $\propto \varepsilon^{4}$ (in the PDOS model function (7)), to the harmonic lattice heat capacities are generally given by the integrals (10). Yet, in order to avoid, henceforth, the corresponding integration procedures also within the frame of least-mean-square fitting processes (like those ones performed in Section 4), it is desirable to substitute the corresponding integrals by appropriate integral-free formulas. This substitution can be immediately performed, for the $\kappa_{C k=1}\left(x_{1}(T)\right)$ function (of Debye type) at least, on the basis of the results obtained in Section A.1. Actually, 
by comparing the integral representations (10) for the $\kappa_{C k=1}\left(x_{1}(T)\right)$ function

$$
\begin{aligned}
\kappa_{C 1}\left(x_{1}\right) & =\frac{3}{x_{1}^{3}} \int_{0}^{x_{1}} d x \frac{x^{4} e^{x}}{\left(e^{x}-1\right)^{2}} \\
& =\frac{3}{4 x_{1}^{3}} \int_{0}^{x_{1}} d x \frac{x^{4}}{(\sinh (x / 2))^{2}}
\end{aligned}
$$

with the integral representations (A.1) and (A.5) for Debye's normalized heat capacity function, $\kappa_{D}\left(x_{D}\right)$, we see that the only difference consists in different limits of integration (and the respective denominators in prefactors), namely, $x_{1}(T) \equiv$ $\Theta_{1} / T$ (in (A.13)), instead of $x_{D}(T) \equiv \Theta_{D} / T$ (in Section A.1). Thus one can directly use the final formulas derived in Section A.1 for the $\kappa_{D}\left(x_{D}(T)\right)$ dependence (like (A.4) and (A.11)) by making but the corresponding substitutions of the characteristic (dimensionless) phonon versus lattice temperature ratios, $x_{D}(T) \rightarrow x_{1}(T)$. Accordingly, the low- and high-temperature branches of the $\kappa_{C 1}\left(x_{1}(T)\right)$ function are explicitly given by

$$
\begin{gathered}
\kappa_{C 1 l}^{n_{D}}\left(x_{1}\right)=\frac{4 \pi^{4}}{5 x_{1}^{3}}-\frac{3 x_{1}}{e^{x_{1}}-1} \\
-12 x_{1} \sum_{n=1}^{n_{D}(\rightarrow \infty)} e^{-n x_{1}}\left(\frac{1}{n x_{1}}+\frac{3}{\left(n x_{1}\right)^{2}}+\frac{6}{\left(n x_{1}\right)^{3}}\right. \\
\left.+\frac{6}{\left(n x_{1}\right)^{4}}\right), \quad \\
\kappa_{C 1 h}^{m_{R}}\left(x_{1}\right)=\left[1+\sum_{m=1}^{m_{R}(\rightarrow \infty)} c_{2 m}^{D R} x_{1}^{2 m}\right]^{-1}=\left[1+\frac{x_{1}^{2}}{20}\right. \\
\left.+\frac{x_{1}^{4}}{1400}+\frac{x_{1}^{6}}{648000}-\frac{73 x_{1}^{8}}{3492720000}+\cdots\right]^{-1},
\end{gathered}
$$

in analogy to (A.4) and (A.11), respectively. An especially useful approximate version of the latter expansion (see (A.15)) is due again to a cut just after the $x_{1}^{14}$ term (i.e., for $m_{R}=$ $7)$. Observing further that the contributions of components $\kappa_{C 1}\left(x_{1}\right)$ of Debye type (as well as the contributions of the components $\kappa_{C 2}\left(x_{1}\right)$ of non-Debye type; see below) to the complete $\kappa_{h}(T)$ function (see (9)) are usually very small (owing to their relatively small weights, $w_{C k} \ll 1$; cf. Table 1 ), it used to be sufficient for practical applications to determine only the first 4 significant figures of these functions. We have found that this reduced degree of precision is achieved when we cut the low-temperature series expansion (A.14) just after the $n_{D}=1$ term. Consequently, we have used for the $\kappa_{C 1}\left(x_{1}\right)$ component (in Section 4) an approximation of the form

$$
\begin{aligned}
\kappa_{\mathrm{Cl}}^{\text {appr. }}\left(x_{1}\right) \longrightarrow \min \left\{\kappa_{\mathrm{C} 1 h}^{m_{R}=7}\left(x_{1}\right), \kappa_{\mathrm{C} 1 l}^{n_{D}=1}\left(x_{1}\right)\right\} & \\
& \text { for } 0 \leq x_{1} \leq 10,
\end{aligned}
$$

in combination with $\kappa_{C 1}^{\text {appr. }}\left(x_{1}\right) \rightarrow \kappa_{C 1 l}^{n_{D}=1}\left(x_{1}\right)$, for $10 \leq x_{1}<$ $\infty$. We see from the upper inset of Figure 1 (in Section 3) that the respective crossing point between the $\kappa_{C 1 h}^{m_{R}=7}\left(x_{1}\right)$ and $\kappa_{C 1 l}^{n_{R}=1}\left(x_{1}\right)$ branches is located at $x_{c 1(D)}=6.5135$, where the deviation of the approximate values, $\kappa_{C 1}^{\text {appr. }}\left(x_{c 1(D)}\right)$ (due to (A.16)) from the exact $\kappa_{C 1}\left(x_{c 1(D)}\right)$ value (A.13), reaches a (maximum) value of $0.748 \times 10^{-4}$.

Consider finally the associated non-Debye function, $\kappa_{C k=2}\left(x_{1}(T)\right)$ (see $(10)$ ), whose dependence on $x_{1}(T)$ was described hitherto by the respective integral representations

$$
\begin{aligned}
\kappa_{\mathrm{C} 2}\left(x_{1}\right) & =\frac{5}{x_{1}^{5}} \int_{0}^{x_{1}} d x \frac{x^{6} e^{x}}{\left(e^{x}-1\right)^{2}} \\
& =\frac{5}{4 x_{1}^{5}} \int_{0}^{x_{1}} d x \frac{x^{6}}{(\sinh (x / 2))^{2}} .
\end{aligned}
$$

Comparing the latter with (A.13) we see that the difference consists in different prefactors (i.e., $5 / x_{1}^{5}$, instead of $3 / x_{1}^{3}$ ) in combination with a higher-order power of the integration variable (i.e., $x^{6}$, instead of $x^{4}$ ), in the integrand of (A.17) versus (A.13). These are the causes of a pronounced qualitative difference of the $x_{1}$-dependence of the $\kappa_{C 2}\left(x_{1}\right)$ versus $\kappa_{C 1}\left(x_{1}\right)$ curve (cf. Figure 1, in Section 3).

A corresponding high-accuracy series expansion for the $\kappa_{C 2 l}\left(x_{1}\right)$ dependence can be derived, again, via several subsequent integrations by parts of the first integral version in (A.17) (in analogy to the derivation of the $\kappa_{D l}^{n_{D}}\left(x_{1}\right)$ series expansion (see (A.4)) from the original integral form (see (A.1)). In this way we have obtained for the low-temperature branch of the $\kappa_{\mathrm{C} 2}\left(x_{1}\right)$ function (see (A.17)) an expansion of the form

$$
\begin{aligned}
& \kappa_{C 2 l}^{n_{N}}\left(x_{1}\right)=\frac{80 \pi^{6}}{21 x_{1}^{5}}-\frac{5 x_{1}}{e^{x_{1}}-1} \\
& -30 x_{1} \sum_{n=1}^{n_{N}(\rightarrow \infty)} e^{-n x_{1}}\left(\frac{1}{n x_{1}}+\frac{5}{\left(n x_{1}\right)^{2}}+\frac{20}{\left(n x_{1}\right)^{3}}\right. \\
& \left.+\frac{60}{\left(n x_{1}\right)^{4}}+\frac{120}{\left(n x_{1}\right)^{5}}+\frac{120}{\left(n x_{1}\right)^{6}}\right) .
\end{aligned}
$$

For the complementary high-temperature behaviour one can use, again, the Taylor series expansion (A.6) for the $(\sinh (x / 2))^{-2}$ function in the second version of (A.17). In this way one comes readily to an infinite Taylor series expansion of the form

$$
\begin{aligned}
\kappa_{\mathrm{C} 2 h}^{m_{N}}\left(x_{1}\right) & =1+\sum_{m=1}^{m_{N}(\rightarrow \infty)}(-1)^{m}\left|c_{2 m}^{N}\right| x_{1}^{2 m} \\
& =1+\sum_{m=1}^{m_{N}(\rightarrow \infty)} c_{2 m}^{N} x_{1}^{2 m}
\end{aligned}
$$

where the respective expansion coefficients for the individual even-order $x_{1}^{2 m}$ power terms (with alternating signs), $c_{2 m}^{N}=$ $(-1)^{m}\left|c_{2 m}^{N}\right|$, are given by

$$
c_{2 m}^{N}=(-1)^{m} \frac{5(2 m-1)\left|B_{2 m}\right|}{(2 m+5) \cdot(2 m) !}, \quad m=1,2,3, \ldots
$$


Accordingly, one finds the numerical values of the expansion coefficients, $c_{2 m}^{N}$, for the first eight $x_{1}^{2 m}$ power terms to be explicitly given by the following fractions:

$$
\begin{aligned}
c_{2}^{N} & =\frac{-5}{84}, \\
c_{4}^{N} & =\frac{1}{432}, \\
c_{6}^{N} & =\frac{-5}{66528}, \\
c_{8}^{N} & =\frac{1}{449280}, \\
c_{10}^{N} & =\frac{-1}{15966720}, \\
c_{12}^{N} & =\frac{691}{404190259200}, \\
c_{14}^{N} & =\frac{-1}{21842472960}, \\
c_{16}^{N} & =\frac{3617}{298777439600400} .
\end{aligned}
$$

However, due to the alternating signs of the $c_{2 m}^{N}$ coefficients (A.21), this high-temperature expansion (see (A.19)) turned out to be, again, a rather slowly converging one (in analogy to the conventional high-temperature expansion (see (A.7)) for the Debye function).

We have found that a considerably faster convergence can be achieved also for the latter case when we change from the original $\kappa_{C 2 h}^{m_{N}}\left(x_{1}\right)$ expansion (see (A.19)) to an associated Taylor series for reciprocal $\kappa_{\mathrm{C} 2 h}^{-1}$ values, $\kappa_{\mathrm{C} 2 \mathrm{Rh}}\left(x_{\mathrm{D}}\right) \equiv$ $1 / \kappa_{C 2 h}\left(x_{D}\right)$. Performing the corresponding transformation of the expansion coefficients, $\left\{c_{2 m}^{N}\right\} \rightarrow\left\{c_{2 m}^{N R}\right\}$ (in analogy, again, to the transformation procedure sketched in Section 4.5.4 of [91]), we came to a Taylor series expansion for the reciprocal values, $\kappa_{C 2 R h}\left(x_{D}\right)$, of the form

$$
\begin{aligned}
\kappa_{C 2 R h}^{m_{R}}\left(x_{1}\right) & =1+\sum_{m=1}^{m_{R}(\rightarrow \infty)} c_{2 m}^{N R} x_{1}^{2 m} \\
& =1+\frac{5 x_{1}^{2}}{84}+\frac{13 x_{1}^{4}}{10584}+\frac{205 x_{1}^{6}}{19559232}+\cdots .
\end{aligned}
$$

Note that a list of the 8 lowest-order expansion coefficients, $c_{2 \leq 2 m \leq 16}^{N R}$, is given in Table 6. Accordingly, the $\kappa_{C 2 h}^{m_{N}}\left(x_{1}\right)$ function in question can be represented, alternatively, in the form of the reciprocal of (A.22); that is, explicitly

$$
\begin{aligned}
\kappa_{C 2 h}^{m_{R}}\left(x_{1}\right) & =\left[1+\sum_{m=1}^{m_{R}(\rightarrow \infty)} c_{2 m}^{N R} x_{1}^{2 m}\right]^{-1} \\
& =\left[1+\frac{5 x_{1}^{2}}{84}+\frac{13 x_{1}^{4}}{10584}+\frac{205 x_{1}^{6}}{19559232}+\cdots\right]^{-1} .
\end{aligned}
$$

One can recognize the cause of significantly improved convergence properties of the latter series expansion (see
(A.23)), in comparison with (A.19), to be due, again, to the qualitatively different constellations of both signs and magnitudes of respective expansions coefficients. Comparing in particular the magnitudes of the original with the transformed expansions coefficients, that is, the $\left|c_{2 m}^{N}\right|$ values (in (A.21)) with the $\left|c_{2 m}^{N R}\right|$ values (listed in Table 6), we see that the latter are decreasing much more rapidly with increasing order $(2 m=2,4,6, \ldots)$ of the individual $x^{2 m}$ power terms. In particular we see that, for example, for $m=8$, the corresponding coefficient, $c_{16}^{N R}=5.356 \times 10^{-17}$ (in (A.23)); cf. Table 6), is by more than 5 orders of magnitude smaller than its counterpart, $c_{16}^{N}=3617 / 298777439600400=1.211 \times 10^{-11}$, due to the original high-temperature Taylor series expansion (A.19). The unusually rapid decrease of magnitudes of the transformed coefficients, $\left|c_{2 m}^{N R}\right|$, with increasing order of the $x_{1}^{2 m}$ power terms is the main reason of significantly improved convergence properties of the high-temperature Taylor series expansion, $\kappa_{C 2 h}^{m_{R}}\left(x_{1}\right)\left(\right.$ see $\left(\right.$ A.23)), for the $\kappa_{C 2}\left(x_{1}\right)$ function (see (A.19)) of non-Debye type.

An especially convenient version of the latter expansion within the present context has been found to be given by a cut just after the $x_{1}^{12}$ term (i.e., for $m_{R}=6$ ). Furthermore it turns out to be sufficient to limit the associated low-temperature expansion (see (A.18)) to the lowest order $\left(n_{N}=1\right)$. Thus we can combine the couple of the corresponding $\kappa_{C 2 h}^{m_{R}=6}\left(x_{1}\right)$ and $\kappa_{C 2 l}^{n_{N}=1}\left(x_{1}\right)$ branches to provide a reasonable approximation for the $\kappa_{\mathrm{C} 2}\left(x_{1}\right)$ function (see (A.17)) of the form

$$
\begin{aligned}
\kappa_{\mathrm{C} 2}^{\text {appr. }}\left(x_{1}\right) \longrightarrow \min \left\{\kappa_{\mathrm{C} 2 h}^{m_{R}=6}\left(x_{1}\right), \kappa_{\mathrm{C} 2 l}^{n_{N}=1}\left(x_{1}\right)\right\} & \\
& \text { for } 0 \leq x_{1} \leq 10,
\end{aligned}
$$

in combination with $\kappa_{\mathrm{C} 2}^{\text {appr. }}\left(x_{1}\right) \rightarrow \kappa_{\mathrm{C} 2 l}^{n_{N}=1}\left(x_{1}\right)$, for $10 \leq x_{1}<\infty$.

We see from the lower inset of Figure 1 (in Section 3) that the respective crossing point between the $\kappa_{C 2 h}^{m_{R}=6}\left(x_{1}\right)$ and $\kappa_{C 2 l}^{n_{N}=1}\left(x_{1}\right)$ branches is located at $x_{c 2(N)}=7.0962$, where the deviation of the approximate values $\kappa_{C 2}^{\text {appr. }}\left(x_{c 2(N)}\right)$ (due to (A.24)) from the exact $\kappa_{\mathrm{C} 2}\left(x_{c 2(N)}\right)$ value (see (A.13)) reaches a (maximum) value of $1.306 \times 10^{-4}$.

\section{B. Integral-Free Formulas for the Inverse Debye Function}

Let us now study in due detail the hitherto almost completely ignored (though practically highly important) inverse problem of devising appropriate, integral-free formulas for the dependence of the upper limits of integration, $x_{D}(T)=$ $\Theta_{D}(T) / T$ (in (A.1)), on the given magnitudes of capacity ratios, $\kappa(T)=C_{p}(T) / C_{V h}(\infty)$.

B.1. Basic Models. For very low (liquid-helium-hydrogen) temperatures, the first term, $\kappa_{D l}^{\lim }\left(x_{D}\right)=4 \pi^{4} / 5 x_{D}^{3}$, in the low-capacity expansion (A.4) is the strongly dominating one. Inverting this asymptotic relationship between $\kappa$ and $x_{D}$ one obtains readily for the corresponding asymptotic 
dependence of $x_{D}$ on $\kappa$ the simple (cubic root) expression formula

$$
x_{D}(\kappa \longrightarrow 0) \longrightarrow x_{D}^{l_{0}}(\kappa)=\sqrt[3]{\frac{4 \pi^{4}}{5 \kappa}} .
$$

Yet, a comparison (in Figure 9) of the asymptotic (dotted) $x_{D}^{l_{0}}(\kappa)$ curve with the exact $x_{D}(\kappa)$ dependence (solid curve) shows that the deviations, $x_{D}^{l_{0}}(\kappa)-x_{D}(\kappa)>0$, are smaller than $1 \%$ only for very low magnitudes of heat capacities, namely, $0<\kappa<0.03$ (cf. the upper inset of Figure 9). This corresponds to a confinement of the applicability of (B.1) to a limiting region of relatively high $x_{D}$ values $\left(x_{D}>14\right.$; see also $[3,13]$ and cf. Table 7$)$. At the same time it is clear that, for higher $\kappa$ values, it is impossible to derive a more general $x_{D}(\kappa)$ formula directly from Debye's rather complex low-capacity (low-temperature) expression [1-3], $\kappa_{D l}^{n_{D}}\left(x_{D}\right)$ (see (A.4)).

In order to come to a practicable algebraic expression for the qualitatively different $x_{D}(\kappa)$ behaviour adopted at sufficiently high $\kappa$ values (including the $\kappa \rightarrow 1$ limit) we start from the unprecedented Taylor series expansion for reciprocal values $\left(\kappa\left(x_{D}\right)\right)^{-1}=\kappa_{D R h}^{m_{R}}\left(x_{D}\right)$ (see (A.10)); that is,

$$
\kappa^{-1}=1+\frac{x_{D}^{2}}{20}+\frac{x_{D}^{4}}{1400}+\frac{x_{D}^{6}}{648000}-\cdots .
$$

Limiting ourselves, for convenience, to the two lowestorder $x_{D}^{2 n}$ terms (which corresponds to the limitation of the summation in (A.10) to $m_{R}=2$ ), we readily obtain via a solution of the respective quadratic equation, $z_{D}^{2} / 1400+$ $z_{D} / 20=\kappa^{-1}-1$ (where $z_{D} \equiv x_{D}^{2}$ ), for the dependence of $x_{D} \equiv \sqrt[2]{z_{D}}$ on $\kappa^{-1}$ in exact way a simple algebraic expression of the form [15]

$$
x_{D}^{h_{0}}(\kappa)=\sqrt[2]{-35+5 \cdot \sqrt[2]{49+56 \cdot\left(\kappa^{-1}-1\right)}} .
$$

This highly useful approximation for the high-capacity (high-temperature) dependence, $x_{D}^{h}(\kappa)$, had already preliminarily been used for some numerical comparisons with the exact $x_{D}(\kappa)$ dependence in our preceding paper [15] (though without indicating, there, its actual origin; cf. Eq. (26) in [15]). Now we have displayed, within the frame of the closely interrelated Appendices A and B, the underlying analytical basis. Furthermore we would like to note that the behaviour of the $x_{D}^{h_{0}}(\kappa)$ function close to the upper limit, $\kappa \rightarrow 1$, follows readily from (B.3) to tend to the asymptote $x_{D}^{h_{0}}(\kappa \rightarrow 1) \rightarrow$ $\sqrt[2]{20 \cdot\left(\kappa^{-1}-1\right)}$ (in accordance with Eq. (27) in [15]).

The whole $x_{D}^{h_{0}}(\kappa)$ dependence (B.3) is represented by the dashed curve (labelled by $h_{0}\left(m_{R}=2\right)$ ) in Figure 9 . Within the large scale representation, at least, this $x_{D}^{h_{0}}(\kappa)$ curve appears to be almost indistinguishable from the exact (solid) $x_{D}(\kappa)$ curve, throughout a very large interval of $\kappa$-values $(0.05<$ $\kappa \leq 1$ ). On the other hand we see from the upper inset (to Figure 9) that the approximate $x_{D}^{h_{0}}(\kappa)$ curve shows within this interval deviations, $\delta x_{D} / x_{D}$, from the exact $x_{D}(\kappa)$ curve up to about $0.6 \%$.

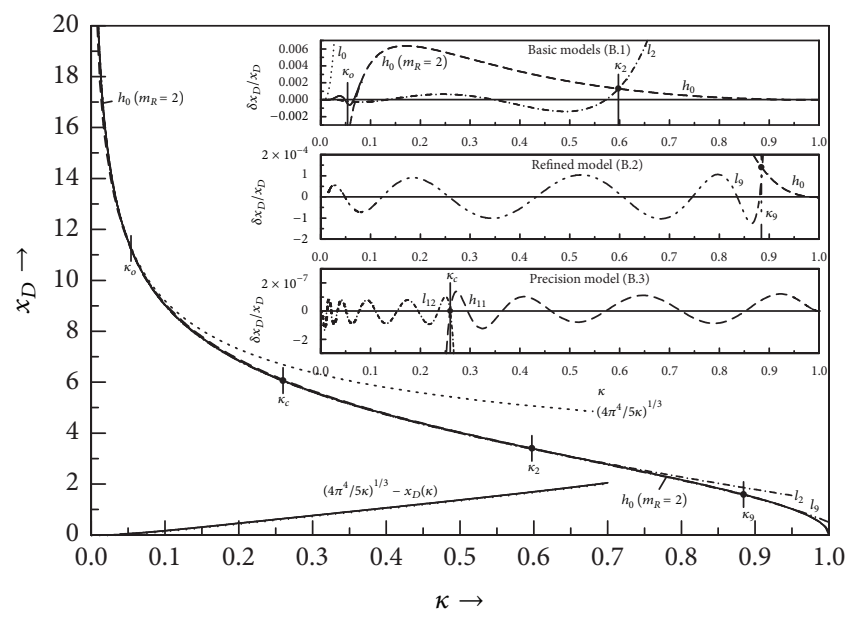

Figure 9: Exact dependence (-) of the characteristic Debye variable $x_{D}\left(=\Theta_{D}(T) / T\right)$ on the relative (normalized) magnitudes of heat capacities, $\kappa\left(=C_{p}(T) / C_{V h}(\infty)\right)$, in comparison with the low- $\kappa$ asymptote, $x_{D}^{l_{0}}(\kappa)=\left(4 \pi^{4} / 5 \kappa\right)^{1 / 3}(\cdots$; see (B.1)), and the characteristic high- $\kappa$ approximation, $x_{D}^{h_{0}}(\kappa)(-----$; see (B.3)). Considerable reductions of maximum deviations to $\pm 0.13 \%$ (see the upper inset) or $\pm 0.0153 \%$ (see the middle inset) are achieved by means of combinations of two more elaborate, qualitatively different versions of low-to-intermediate- $\kappa$ approximations, $x_{D}^{l_{2}}\left(0 \leq \kappa \leq \kappa_{2}\right)$ $\left(-\cdot-\cdot-\cdot ;\right.$ see (B.5a) and (B.5b)) and $x_{D}^{l_{9}}\left(0 \leq \kappa \leq \kappa_{9}\right)(--\cdot--\cdot-$; see (B.6)), in combination with the complementary high- $\kappa$ curve sections, that is, $x_{D}^{h_{0}}\left(\kappa_{2} \leq \kappa \leq 1\right)$ or $x_{D}^{h_{0}}\left(\kappa_{9} \leq \kappa \leq 1\right)$, respectively. From the lower inset we see that the residual deviations of the complementary high-precision formulas $x_{D}^{l_{12}}\left(0 \leq \kappa \leq \kappa_{c}\right)$ (see (B.8)) and $x_{D}^{h_{11}}\left(\kappa_{c} \leq \kappa \leq 1\right)$ (see (B.9)) are throughout smaller than $\pm 0.000016 \%$.

Considering in more detail the characteristic deviations of both the $x_{D}^{l_{0}}(\kappa)$ curve (see (B.1)) and of the $x_{D}^{h_{0}}(\kappa)$ curve (see (B.3)) from the exact $x_{D}(\kappa)$ dependence in low-capacity region $(0<\kappa<0.07)$ we see that they have different signs, and their magnitudes are reaching the same order (of about $\pm 1.5 \%$ ) in the vicinity of $\kappa \approx 0.035$. For such a constellation of noncrossing curve branches it is possible to achieve a significant reduction (partial mutual compensation) of deviations via a connection of the two complementary curve branches, $x_{D}^{l_{0}}(\kappa)$ and $x_{D}^{h_{0}}(\kappa)$, by an appropriate junction formula of type

$$
\tilde{x}_{D}(\kappa)=\frac{x_{D}^{h_{0}}(\kappa)+\exp \left(\left(\kappa_{o}-\kappa\right) / \Delta \kappa\right) x_{D}^{l_{0}}(\kappa)}{1+\exp \left(\left(\kappa_{o}-\kappa\right) / \Delta \kappa\right)} .
$$

An optimal approach of $\tilde{x}_{D}(\kappa)$ to the exact $x_{D}(\kappa)$ function, within the interval $0<\kappa<0.1$, has been found via a corresponding least-mean square fitting process. This led to the following couple of junction parameter values:

$$
\begin{aligned}
\kappa_{o} & =0.054, \\
\Delta \kappa & =0.006 .
\end{aligned}
$$

The respective $\tilde{x}_{D}(\kappa)$ curve is represented in the upper inset of Figure 9 by the slightly oscillating solid curve section, 
for $\kappa<0.07$ (corresponding to an $x_{D}$-region of $x_{D}>10$ ). In view of the resulting limitation of residual deviations to on order of $\pm 0.06 \%$ (within the interval $0<\kappa<0.07$ ) it is obvious that the adoption of the junction formula, $\tilde{x}_{D}(\kappa)$ (see (B.4a) and (B.4b)), effectuates here a significantly closer approach to the exact $x_{D}(\kappa)$ curve than their individual components per se, $x_{D}^{l_{0}}(\kappa)($ see $(B .1))$ and $x_{D}^{h_{0}}(\kappa)$ (see (B.3)). On the other hand we see also from the upper inset of Figure 9 that, outside this relatively narrow low-capacity interval (i.e., for $\kappa>0.07)$, the junction formula, $\widetilde{x}_{D}(\kappa)$ (see (B.4a) and (B.4b)), is still implying deviations from the exact $x_{D}(\kappa)$ curve up to about $0.6 \%$ (owing to the asymptotic approach $\tilde{x}_{D}(\kappa) \rightarrow$ $x_{D}^{h_{0}}(\kappa)$, which is implied by the actual junction parameter constellation, $\kappa_{o}$ and $\Delta \kappa(B .4 b)$, in (B.4a)).

An important chance for significant improvements of the analytical description of the $x_{D}(\kappa)$ dependence within the middle region $(0.1<\kappa<0.7)$ is provided by the unfamiliar observation that the difference, $x_{D}^{l_{0}}(\kappa)-x_{D}(\kappa) \geq 0$, between the $\kappa \rightarrow 0$ asymptote, $x_{D}^{l_{0}}(\kappa)=\sqrt[3]{4 \pi^{4} / 5 \kappa}$ (dotted curve), and the exact $x_{D}(\kappa)$ dependence (solid curve) corresponds, roughly, to a linear dependence on $\kappa$. This peculiarity is apparent from the respective $\sqrt[3]{4 \pi^{4} / 5 \kappa}-x_{D}(\kappa) \geq 0$ curve (as shown in the lower part of Figure 9). Estimating the slope of this curve (e.g., within an interval of $0.2<\kappa<0.5$ ) we have obtained an average value of $d\left(x_{D}^{l_{0}}(\kappa)-x_{D}(\kappa)\right) / d \kappa \cong$ 3.036. At the same time we see (from Figure 9) that the slope of this curve decreases monotonically, with decreasing $\kappa$, and finally tends to 0 in the $\kappa \rightarrow 0$ limit. This behaviour of the slope can be simulated, for example, by some "slope reduction factor" of exponential type, $\exp (-p / \kappa)$, so that the difference in question has a chance to be reasonably approximated by a function of type $g \kappa \exp (-p / \kappa)$ (where the factor $g$ should be approximately equal to the effective slope (quoted above) for the middle region). Accordingly, we choose for an approximate simulation of the $x_{D}(\kappa)$ dependence in the region of low-to-intermediate $\kappa$ values an effective 2-parameter formula of type

$$
x_{D}^{l_{2}}(\kappa)=\sqrt[3]{\frac{4 \pi^{4}}{5 \kappa}}-g \kappa \exp \left(-\frac{p}{\kappa}\right) .
$$

Performing a least-means square process of the exact $x_{D}(\kappa)$ curve by means of this approximate 2-parameter formula (B.5a), within the range $0<\kappa<0.63$, we have obtained the parameter values

$$
\begin{aligned}
& g=3.145972, \\
& p=0.07037359 .
\end{aligned}
$$

This extended low- $\kappa$ curve branch, $x_{D}^{l_{2}}(\kappa)$ (see (B.5a) and (B.5b)), crosses the prefabricated high- $\kappa$ curve branch, $x_{D}^{h_{0}}(\kappa)$ (see (B.3)), at the point $\kappa_{2}=0.5975$ (see the upper inset of Figure 9). At this point the deviations of both approximate curves from the exact one are found to amount to about $0.13 \%$. This maximum overestimation at the crossing point, $\kappa_{2}$, is seen to be of the same order as the maximum underestimation of the exact $x_{D}(\kappa)$ function by the $x_{D}^{l_{2}}(\kappa)$ function (see (B.5a) and (B.5b)), in the vicinity of $\kappa \approx 0.5$. Henceforth, by switching from the extended low- $\kappa$ formula $x_{D}^{l_{2}}(\kappa)$ (see (B.5a) and (B.5b)) to the high- $\kappa$ formula $x_{D}^{h_{0}}(\kappa)$ (see (B.3)) just at the crossing point, $\kappa_{2}=0.5975$, we are obtaining a complete sequence of approximate $x_{D}(0 \leq \kappa \leq 1)$ values; the maximum deviations from the exact ones are not exceeding an order of $\pm 0.13 \%$. Such approximate results may already appear to be largely acceptable with respect to various practical applications.

In this connection we would still like to note that, even within the low-temperature region $(0<T<300 \mathrm{~K})$, the uncertainties of experimentally measured heat capacities, $C_{p}(T)$, used to be of order 0.5 to $1 \%$. Thus the inherent experimental uncertainties used to have a significantly stronger influence on residual uncertainties of final results than the computational inaccuracies that are involved by the use of a couple of complementary approximation formulas, like $x_{D}^{l_{2}}\left(0<\kappa \leq \kappa_{2}\right)$ (see (B.5a) and (B.5b)) in combination with $x_{D}^{h_{0}}\left(\kappa_{2} \leq \kappa \leq 1\right)(\operatorname{see}($ B.3 $))$.

B.2. Refined Model. A significant refinement of the analytical model for low-to-intermediate heat capacities can be achieved when we take properly into account that the slope of the difference, $\sqrt[3]{4 \pi^{4} / 5 \kappa}-x_{D}(\kappa) \geq 0$, tends to be slightly increasing in the region $\kappa>0.5$ (cf. the corresponding curve in the lower part of Figure 9). One can readily simulate these successively increasing deviations from linearity by including a series of higher-order power terms, $\kappa^{n}(n=2,3, \ldots)$, into (B.5a). Limiting ourselves here to a series of 7 additional power terms, and adopting a unique slope reduction factor for the whole power series, we can well represent the $x_{D}^{l}(\kappa)$ dependence by a significantly refined 9-parameter expression of the form

$$
x_{D}^{l_{9}}(\kappa)=\sqrt[3]{\frac{4 \pi^{4}}{5 \kappa}}-\exp \left(-\frac{p}{\kappa}\right) \sum_{n=1}^{8} g_{n} \kappa^{n}
$$

Performing a least-means square fitting process of the exact $x_{D}(\kappa)$ curve by means of this 9-parameter formula (B.6) within the range $0<\kappa<0.89$ we have obtained the parameter values listed in the first column of Table 8 . The respective $x_{D}^{l_{9}}(\kappa)$ curve is seen to cross the prefabricated high- $\kappa$ curve branch, $x_{D}^{h_{0}}(\kappa)$ (see (B.3)), at the point $\kappa_{9}=0.8844$ (see the middle inset of Figure 9). At this point the deviations of the two complementary (approximate) curve branches, $x_{D}^{l_{9}}(\kappa)$ and $x_{D}^{h_{0}}(\kappa)$, from the exact $x_{D}(\kappa)$ curve are seen to be of order $0.015 \%$. This slight overestimation at the crossing point is seen to be of the same order of magnitude as the maximum deviations, $\left(\delta x_{D} / x_{D}\right)_{\max } \approx \pm 0.015 \%$, of the significantly refined 9-parameter formula, $x_{D}^{l_{9}}(\kappa)$ (see (B.6)) from the exact $x_{D}(\kappa)$ dependence throughout the whole interval of its possible applicability, $0 \leq \kappa \leq \kappa_{9}$. Consequently, by switching from the latter to the prefabricated high $-\kappa$ formula, $x_{D}^{h_{0}}(\kappa)($ see $(B .3))$ just at the respective crossing point, $\kappa_{9}=$ 0.8844 , one is coming to a significantly refined sequence of approximate $x_{D}(0 \leq \kappa \leq 1)$ values; the maximum deviations 
TABLE 8: Fitted values of the model-specific coefficients involved by the refined low-to-intermediate capacity $x_{D}(\kappa)$ formula, $x_{D}^{l_{9}}\left(0<\kappa \leq \kappa_{9}\right)$ (B.6), and for the couple of complementary high-accuracy low- and high-capacity Debye temperature formulas, $x_{D}^{l_{12}}\left(0<\kappa \leq \kappa_{c}\right)($ B.8) and $x_{D}^{h_{11}}\left(\kappa_{c} \leq \kappa \leq 1\right)($ B.9), which have been determined by least-mean-square fittings of a sufficiently comprehensive set of couples of high-accuracy $\kappa$ versus $x_{D}$ curve points (in accordance with Table 7 ).

\begin{tabular}{lcr}
\hline$x_{D}^{l_{9}}(\kappa)($ see $($ B.6 $))$ & $x_{D}^{l_{12}}(\kappa)($ see $(\mathrm{B} .8))$ & $x_{D}^{h_{11}}(\kappa)(\mathrm{see}(\mathrm{B} .9))$ \\
$0<\kappa \leq \kappa_{9}=0.8844$ & $0<\kappa \leq \kappa_{c}=0.260$ & $0.260=\kappa_{c} \leq \kappa \leq 1$ \\
\hline$p=0.071797185$ & $a_{2}=2.8743354 \times 10^{-1}$ & $b_{2}=6.0777673 \times 10^{-3}$ \\
$g_{1}=3.042348$ & $a_{3}=1.1377662 \times 10^{-1}$ & $b_{3}=5.1466371 \times 10^{-3}$ \\
$g_{2}=1.816742$ & $a_{4}=-9.5999179 \times 10^{-2}$ & $b_{4}=-6.8442924 \times 10^{-3}$ \\
$g_{3}=-6.892814$ & $a_{5}=4.3062699 \times 10^{-2}$ & $b_{5}=2.6051894 \times 10^{-2}$ \\
$g_{4}=6.954053$ & $a_{6}=-4.1060421 \times 10^{-3}$ & $b_{6}=-3.6011454 \times 10^{-2}$ \\
$g_{5}=5.456090$ & $a_{7}=-1.1955013 \times 10^{-3}$ & $b_{7}=3.6169365 \times 10^{-2}$ \\
$g_{6}=-11.20018$ & $a_{8}=4.9953204 \times 10^{-4}$ & $b_{8}=-3.7204523 \times 10^{-2}$ \\
$g_{7}=2.022044$ & $a_{9}=-6.2681506 \times 10^{-5}$ & $b_{9}=1.6804524 \times 10^{-2}$ \\
$g_{8}=2.824376$ & $a_{10}=3.3449835 \times 10^{-6}$ & $b_{10}=-3.0967328 \times 10^{-3}$ \\
- & $p=1.2969$ & $b_{11}=5.2219439 \times 10^{-2}$ \\
- & $g_{2}=-3.1541530$ & $b_{12}=-6.2935315 \times 10^{-2}$ \\
- & $g_{3}=8.7184026$ & - \\
\hline
\end{tabular}

from the exact ones are not surpassing the rather narrow inaccuracy range of $\pm 0.015 \%$.

B.3. Precision Model. Notwithstanding the circumstance that the refined model just displayed (within the preceding Section B.2) may already be looked upon as a quite adequate one for most practical applications, it appears useful from theoretical points of view to envisage still the construction of a highly elaborate analytical model, the numerical accuracy of which should be by several orders of magnitude higher than the preceding one. To this end we observe, first of all, that a detailed study of the $\kappa \rightarrow 0$ limiting behaviour of the exact $x_{D}(\kappa)$ dependence does not really show a perfect plateau behaviour (as suggested above by the global exponential slope reduction factors, $\exp (-p / \kappa)$, in (B.5a), (B.5b), and (B.6)). Actually, we have found within a careful numerical analysis that the difference between the $\kappa \rightarrow 0$ asymptote, $x_{D}^{l_{0}}(\kappa)$ (see (B.1)), and the exact $x_{D}(\kappa)$ dependence at $\kappa \ll 0.01$ is tending just to a 5 th-order power dependence, $\left[x_{D}^{l_{0}}(\kappa)-x_{D}(\kappa)\right] \propto$ $\kappa^{5}$. Towards higher $\kappa$ values, the effective power function exponent decreases successively until the difference reaches a nearly linear dependence, in the vicinity of $\kappa \approx 0.2$. We have found that a very fine simulation of this gradual change from a $\kappa^{5}$ to a $\kappa^{1}$ dependence can be achieved via the reciprocal values of a truncated power series consisting of $\kappa^{-5}$ to $\kappa^{-1}$ power terms (including, for convenience, even terms with half integer exponents); that is,

$$
\left[x_{D}^{l_{0}}(\kappa)-x_{D}(\kappa)\right] \longrightarrow\left[\sum_{n=2}^{10} \frac{a_{n}}{\kappa^{n / 2}}\right]^{-1}
$$

within the range $0<\kappa<0.2$.

For the sake of an extension of the applicability of the envisaged high-accuracy formula up to $\kappa \approx 0.25$ (at least) we shall still include (in analogy to (B.6)) a quadratic and a cubic term in combination, again, with an exponential slope reduction factor for these two additional terms. In summary, we shall represent the $x_{D}(\kappa)$ dependence within the region $0<\kappa<0.26$ by the elaborate 12 -parameter formula

$$
\begin{aligned}
x_{D}^{l_{12}}(\kappa)= & \sqrt[3]{\frac{4 \pi^{4}}{5 \kappa}}-\left[\sum_{n=2}^{10} \frac{a_{n}}{\kappa^{n / 2}}\right]^{-1} \\
& -\exp \left(-\frac{p}{\kappa}\right) \sum_{n=2}^{3} g_{n} \kappa^{n} .
\end{aligned}
$$

Performing a least-mean-square fitting process of the exact $x_{D}(\kappa)$ curve by means of this 12 -parameter formula (B.8) within the range $0<\kappa<0.26$ we have obtained the adjusted parameter values $\left(a_{n}, n=2\right.$ to $10, g_{2}, g_{3}$, and $\left.p\right)$ listed in Table 8 . From the lower inset of Figure 9 we see that the still remaining deviations of the respective $x_{D}^{l_{12}}(\kappa)$ curve (see (B.8)) from the exact $x_{D}(\kappa)$ dependence are limited to an order of $\pm 0.000013 \%$. This means an increase of accuracy of numerical results due to (B.8) by 3 orders of magnitude in comparison with (B.6). A set of corresponding high-accuracy $x_{D}^{l_{12}}(\kappa)$ values (for $0 \leq \kappa \leq 0.26$ ) is presented in Table 9 .

In order to achieve a similarly strong increase of accuracy also for the rest interval of intermediate-to-high $\kappa$ values $(0.26 \leq \kappa \leq 1)$ we endow the prefabricated high-capacity formula $x_{D}^{h_{0}}(\kappa)\left(\right.$ B.3) by an appropriate power series of $(1-\kappa)^{n}$ terms ( $n=2$ to 12 ) occurring in the denominator. This model corresponds thus to an 11-parameter expression of the form

$$
x_{D}^{h_{11}}(\kappa)=\frac{\sqrt[2]{-35+5 \cdot \sqrt[2]{49+56 \cdot\left(\kappa^{-1}-1\right)}}}{\left[1+\sum_{n=2}^{12} b_{n}(1-\kappa)^{n}\right]} .
$$




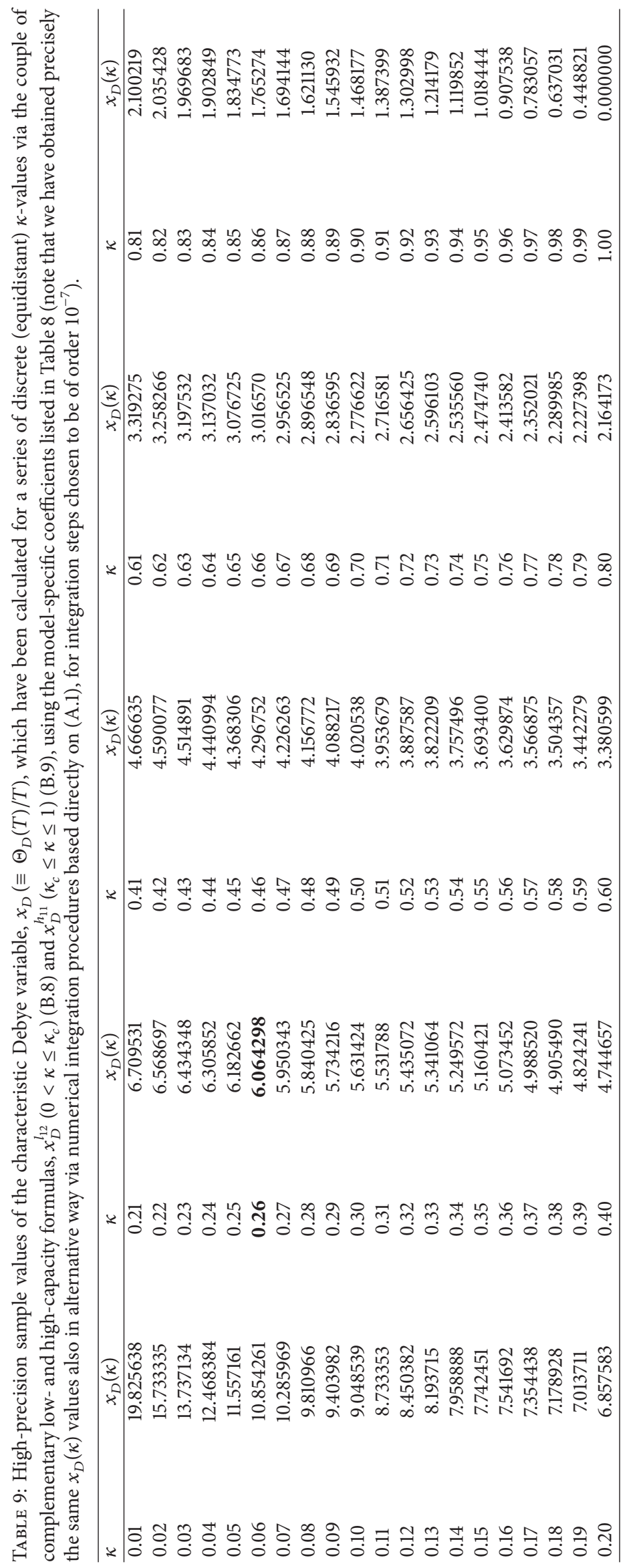


Performing a least-means square fitting process of the exact $x_{D}(\kappa)$ curve by means of this 11-parameter formula (B.9) within the range $0.25<\kappa<1$ we have obtained the adjusted parameter values $\left(b_{n}, n=2\right.$ to 12$)$ listed in Table 8 . From the lower inset of Figure 9 we see that the residual deviations of the respective $x_{D}^{h_{11}}(\kappa)$ curve (see (B.9)) from the exact $x_{D}(\kappa)$ dependence are limited to an order of $\pm 0.000016 \%$ (which are only slightly higher than the residual deviations due to the complementary low-to-intermediate $\kappa$ high-accuracy formula $x_{D}^{l_{12}}(\kappa)$ (see (B.8))). A set of corresponding highaccuracy $x_{D}^{h_{11}}(\kappa)$ values (for $0.26 \leq \kappa \leq 1$ ) is presented in Table 9.

\section{Conventional Estimation Method for True Debye Temperatures}

An effective procedure for determining high-temperature limiting values of the "true" (harmonic-lattice-related) Debye temperatures, $\Theta_{D h}(T \rightarrow \infty)$, on the basis of measured (isobaric) low-temperature heat capacity data, $C_{p}(T) \cong$ $C_{V h}(T)$, had been invented years ago by Barron et al. [16, 17]. A crucial feature of this conventional method is due to the circumstance that, in analogy to the exponential series analysis scheme [54] for heat capacities, its use for analyses of appropriate $\Theta_{D}(T)$ data sets does not assume a preliminary analytical modelling of PDOS spectra functions. The corresponding analysis procedure is based on the observation that certain sections of low-temperature dependencies of the squares, $\left(\Theta_{D h}(T)\right)^{2}$, of "true" Debye temperatures can be generally represented in form of Taylor series expansions involving exclusively even-order power terms of the reciprocal temperature with alternating signs [14-18, 21, 71, 88]; that is, explicitly

$$
\begin{aligned}
& \left(\Theta_{D h}(T)\right)^{2} \\
& \quad=\left(\Theta_{D h}(\infty)\right)^{2}\left(1+\sum_{s=1}^{\infty}(-1)^{s} a_{2 s}\left(\frac{\Theta_{D h}(\infty)}{T}\right)^{2 s}\right) .
\end{aligned}
$$

The symbol $\Theta_{D h}(\infty)$ represents here again the $T \rightarrow \infty$ limit of the "true" (harmonic) Debye temperature, and the expansion coefficients, $a_{2 s}$, used to be positive (and strongly decreasing in magnitude, with increasing order; $1 \gg a_{2} \gg$ $\left.a_{4} \gg a_{6}, \ldots\right)$. The applicability of (C.1) is naturally limited to those regions of (usually relatively low) temperatures where the isochoric/harmonic and isobaric heat capacities are nearly coincident, $C_{V h}(T) \cong C_{p}(T)$, which implies, as assumed, an approximate equality of "true" Debye temperatures with their effective $\left(C_{p}(T)\right.$-related) counterparts, $\Theta_{D h}(T) \cong \Theta_{D}(T)$. This requirement finds its visual realization (within a $\Theta_{D}$ versus $T^{-2}$ representation like Figure 10) in limitations of fitting ranges to those regions where the sequences of the $\Theta_{D}(T)$ points show exclusively concave shapes.

The corresponding material-specific limitations of fitting ranges are indicated in the insets of Figure 10 by vertical (broken) lines and are quoted in Table 10. Accordingly, the upper limits of harmonic lattice behaviour are corresponding

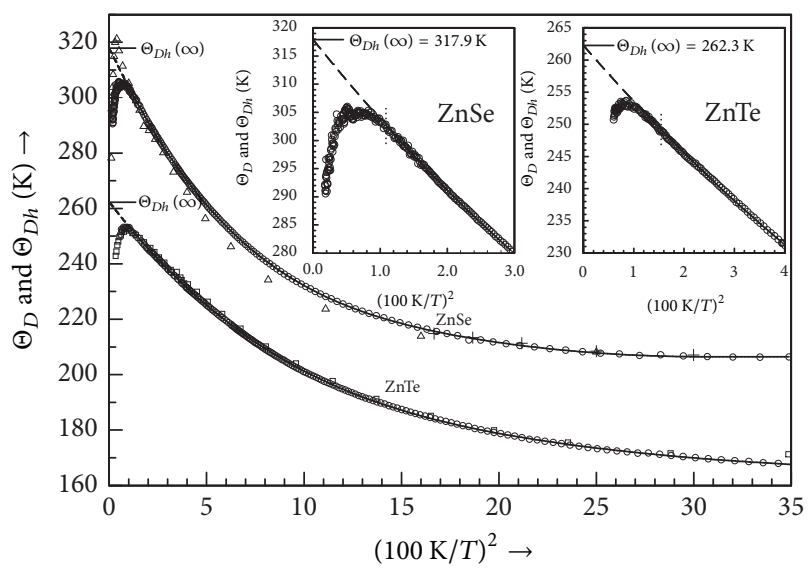

FIGURE 10: Fitting by means of (C.1) of the temperature dependencies of the harmonic sections (solid curves) of Debye temperature data, $\Theta_{D h}(T) \cong \Theta_{D}(T)$, that were directly resulting from the highaccuracy transformations (via (27) or (28)) of the experimental $C_{p}(T)$ data points provided by Kremer et al. ( $\left.\bigcirc[46]\right)$, for natural $\mathrm{ZnSe}$ and $\mathrm{ZnTe}$. The extrapolations of the harmonic (concave) $\Theta_{D h}(T)$ versus $\propto T^{-2}$ curve sections to the $T \rightarrow \infty$ limit are represented by dashed curve sections. For comparison we have also shown the more or less deviating $\Theta_{D}(T)$ data points due to $C_{p}(T)$ data that have been available from earlier studies for ZnSe by Birch $(+[34])$ and Sirota et al. $(\triangle[60])$ and for ZnTe by Gavrichev et al. $(\square[02 \mathrm{G}])$.

to cuts of the relevant $T$-intervals at $T \leq 96 \mathrm{~K}$, for $\mathrm{ZnSe}$, and at $T \leq 80 \mathrm{~K}$, for ZnTe (i.e., at temperatures of the order $(4 / 3) T_{h}$, in both cases; cf. also Figures 2 and 3). Performing hence the least-mean-square fittings of the duly limited series of $\Theta_{D}(T)$ points $(O)$ for $\mathrm{ZnSe}$ and $\mathrm{ZnTe}$ (due to the measured $C_{p}(T)$ data provided by Kremer et al. [46]) via (C.1), we have obtained the respective magnitudes (listed in Table 10) of limiting Debye temperature, $\Theta_{D h}(\infty)$, in combination with material-specific series of even-order expansion coefficients, $a_{2 s}$.

On the basis of these sets of fitted parameter values it is possible to calculate a series of even-order PDOS spectra moments, $\mu_{P}^{(2 s)}$, via the following equations [14]:

$$
\begin{aligned}
\mu_{P}^{(2)} & =\frac{3}{5}\left(\varepsilon_{D h}(\infty)\right)^{2}, \\
\mu_{P}^{(4)} & =\left[\frac{3}{7}+12 a_{2}\right]\left(\varepsilon_{D h}(\infty)\right)^{4}, \\
\mu_{P}^{(6)} & =\left[\frac{3}{9}+\frac{108}{5} a_{2}+\frac{1512}{5} a_{4}\right]\left(\varepsilon_{D h}(\infty)\right)^{6}, \\
\mu_{P}^{(8)} & =\left[\frac{3}{11}+\frac{200}{7} a_{2}+\frac{2160}{7}\left(2 a_{4}+\left(a_{2}\right)^{2}\right)\right. \\
+ & \left.8640 a_{6}\right]\left(\varepsilon_{D h}(\infty)\right)^{8}, \\
\mu_{P}^{(10)} & =\left[\frac{3}{13}+\frac{168}{5} a_{2}+880\left(a_{4}+\left(a_{2}\right)^{2}\right)\right. \\
+ & \left.19008\left(a_{6}+a_{2} a_{4}\right)+266112 a_{8}\right]\left(\varepsilon_{D h}(\infty)\right)^{10}
\end{aligned}
$$


TABLE 10: Limiting values of the "true" (harmonic lattice) Debye temperatures, $\Theta_{D h}(\infty)$, including adjusted magnitudes of several even-order expansion coefficients, $a_{2 s}$, that are representing the weights of the individual $\left(\Theta_{D h}(\infty) / T\right)^{2 s}$ power terms in (C.1). These sets of parameter values have been determined via fittings (shown in Figure 10) of an appropriate selection of usable Debye temperature points, $\Theta_{D h}(T) \cong \Theta_{D}(T)$, implied by the experimental $C_{p}(T)$ data points given by Kremer et al. [46] for natural $\mathrm{ZnSe}$ and ZnTe. Listed are also the magnitudes of the respective (Debyemodel-related) cut-off phonon energies, $\varepsilon_{D h}(\infty) \equiv k_{B} \Theta_{D h}(\infty)$, and several even-order phonon energy values, $\varepsilon_{P}(2 s) \equiv\left(\mu_{P}^{(2 s)}\right)^{1 / 2 s}$ (for $2 s=2$ to 10 ), which are resulting from the corresponding PDOS spectra moments, $\mu_{P}^{(2 s)}$, calculated on the basis of the analytical expressions (C.2a) to (C.2e) (note that the latter equations are exactly corresponding to the series of $\mu_{P}^{(2 s)}$ formulas originally derived within the frame of the supporting information to [21]; see also appendix B of [14] and/or the appendix B of [15]).

\begin{tabular}{lcc}
\hline & ZnSe & ZnTe \\
\hline Intervals (K) & 17 to 96 & 15 to 80 \\
\hline$\Theta_{D h}(\infty)(\mathrm{K})$ & 317.9 & 262.3 \\
$a_{2}$ & $0.9259 \times 10^{-2}$ & $0.9995 \times 10^{-2}$ \\
$a_{4}$ & $0.6895 \times 10^{-4}$ & $0.7851 \times 10^{-4}$ \\
$a_{6}$ & $0.2869 \times 10^{-6}$ & $0.3410 \times 10^{-6}$ \\
$a_{8}$ & $0.6533 \times 10^{-9}$ & $0.7714 \times 10^{-9}$ \\
$a_{10}$ & $\left(0.7135 \times 10^{-12}\right)$ & $\left(0.7038 \times 10^{-12}\right)$ \\
$a_{12}$ & $\left(0.2510 \times 10^{-15}\right)$ & - \\
\hline$\varepsilon_{D h}(\infty)(\mathrm{meV})$ & 27.40 & 22.60 \\
$\varepsilon_{P}(2)(\mathrm{meV})$ & 21.22 & 17.51 \\
$\varepsilon_{P}(4)(\mathrm{meV})$ & 23.48 & 19.45 \\
$\varepsilon_{P}(6)(\mathrm{meV})$ & 24.83 & 20.60 \\
$\varepsilon_{P}(8)(\mathrm{meV})$ & 25.75 & 21.38 \\
$\varepsilon_{P}(10)(\mathrm{meV})$ & 26.42 & 21.95 \\
\hline
\end{tabular}

in complete accordance with the set of $\mu_{P}^{(2 s)}$ formulas originally derived within the supporting information to [21]. Inserting into (C.2a) to (C.2e) the estimated magnitudes (listed in Table 10) for the cut-off phonon energies, $\varepsilon_{D h}(\infty) \equiv$ $k_{B} \Theta_{D h}(\infty)$, and the associated expansion coefficients, $a_{2}$ to $a_{8}$, we have calculated the respective even-order moments, $\mu_{P}^{(2)}$ to $\mu_{P}^{(10)}$. From these moments follow readily the materialspecific magnitudes of the corresponding even-order phonon energies, $\varepsilon_{P}(2 s)=\left(\mu_{P}^{(2 s)}\right)^{1 / 2 s}$. The latter are listed in Table 10 and represented by open squares in Figure 7.

\section{Conflicts of Interest}

The author declares that there are no conflicts of interest regarding the publication of this paper.

\section{Acknowledgments}

The author would like to thank Dr. R. K. Kremer, at the MaxPlanck-Institut für Festkörperphysik, Stuttgart, Germany, for having sent him, in digital form, the experimental heat capacity data published for natural $\mathrm{ZnSe}$ and $\mathrm{ZnTe}$ in [46].

\section{References}

[1] P. Debye, "Zur Theorie der spezifischen Wärmen," Annalen der Physik, vol. 344, no. 14, pp. 789-839, 1912.

[2] E. Schrödinger, "'Spezifische Wärme', in Handbuch der Physik," in Thermische Eigenschaften der Stoffe, C. Drucker, E. Grüneisen, Ph. Kohnstamm et al., Eds., pp. 275-320, Springer-Verlag, Berlin, Heidelberg, 1926.

[3] M. Blackman, "The Specific Heat of Solids," in Encyclopedia of Physics, vol. 3 of part 1: Crystal Physics 1, pp. 325-382, Springer Berlin Heidelberg, Berlin, Heidelberg, 1955.

[4] P. H. Keesom and N. Pearlman, "Low Temperature Heat Capacity of Solids," in Encyclopedia of Physics, S. Flügge, Ed., vol. 14 of Low temperature physics I, pp. 282-337, Springer-Verlag, Berlin-Göttingen-Heidelberg, Berlin, Heidelberg, 1956.

[5] A. J. Dekker, Solid State Physics, Macmillan Education UK, London, 1981.

[6] E. S. Gopal, Specific Heats at Low Temperatures, Springer US, Boston, MA, 1966.

[7] S. Kobe, Crystal Research and Technology, Group III, vols. 17a/b, 22a/b, and 41a/b, Springer-Verlag, Berlin, Germany, 1982.

[8] T. H. Barron and G. K. White, Heat Capacity and Thermal Expansion at Low Temperatures, Springer US, Boston, MA, 1999.

[9] S. Adachi, Handbook on Physical Properties of Semiconductors, vol. vols. 1, 2, and 3, Kluwer Academic Publishers, Boston, Dordrecht, New York, London, 2004.

[10] S. Adachi, Properties of Group-IV, III-V, and II-VI Semiconductors, Wiley Sons Ltd, The Atrium, Southern Gate, Chichester, West Sussex, England, UK, 2005.

[11] U. Piesbergen, "Heat Capacity and Debye Temperatures," in Semiconductors and Semimetals, R. K. Willardson and A. C. Beer, Eds., vol. 2 of Physics of III-V Compounds, Chapter 3, pp. 49-60, Academic Press New York and London, 1966.

[12] J. S. Blakemore, "Semiconducting and other major properties of gallium arsenide," Journal of Applied Physics, vol. 53, no. 10, pp. R123-R181, 1982.

[13] R. Pässler, "Limiting Debye temperature behavior following from cryogenic heat capacity data for group-IV, III-V, and IIVI materials," Physica Status Solidi (B) Basic Research, vol. 247, no. 1, pp. 77-92, 2010.

[14] R. Pässler, "Non-Debye behaviours of heat capacities of cubic IIVI materials," Journal of Physics and Chemistry of Solids, vol. 72, no. 11, pp. 1296-1311, 2011.

[15] R. Pässler, "Non-Debye heat capacity formula refined and applied to GaP, GaAs, GaSb, InP, InAs, and InSb," AIP Advances, vol. 3, no. 8, Article ID 082108, 2013.

[16] T. H. Barron, W. T. Berg, and J. A. Morrison, "The thermal properties of alkali halide crystals. II. Analysis of experimental results," in Proceedings of the Royal Society, vol. 242, pp. 478492, London, UK, 1957.

[17] T. H. Barron, W. T. Berg, and J. A. Morrison, "On the heat capacity of crystalline magnesium oxide," in Proceedings of the Royal Society, vol. 250, pp. 70-83, London, UK, 1959.

[18] P. Flubacher, A. J. Leadbetter, and J. A. Morrison, "The Heat Capacity of Pure Silicon and Germanium and Properties of their Vibrational Frequency Spectra," The Philosophical Magazine, vol. 4, no. 39, pp. 273-294, 1959.

[19] G. Dolling and R. A. Cowley, "The thermodynamic and optical properties of germanium, silicon, diamond and gallium arsenide," Proceedings of the Physical Society, vol. 88, no. 2, article no. 318, pp. 463-494, 1966. 
[20] W. Schnelle and E. Gmelin, "Heat capacity of germanium crystals with various isotopic composition," Journal of Physics Condensed Matter, vol. 13, no. 27, pp. 6087-6094, 2001.

[21] R. Pässler, "Dispersion-related theory for heat capacities of semiconductors," Physica Status Solidi (B) Basic Research, vol. 244, no. 12, pp. 4605-4623, 2007.

[22] U. Piesbergen, "Die durchschnittlichen Atomwärmen der A ${ }^{I I I}$ $\mathrm{B}^{V}$-Halbleiter ALSb, GaAs, GaSb, InP, InAs, InSb und die Atomwärme des Elements Germanium zwischen 12 und 273 -K," Zeitschrift fur Naturforschung - Section A Journal of Physical Sciences, vol. 18, no. 2, pp. 141-147, 1963.

[23] T. C. Cetas, C. R. Tilford, and C. A. Swenson, "Specific heats of $\mathrm{Cu}, \mathrm{GaAs}, \mathrm{GaSb}$, InAs, and InSb from 1 to $30 \circ \mathrm{K}$," Physical Review, vol. 174, no. 3, pp. 835-844, 1968.

[24] A. F. Demidenko, V. I. Koshchenko, A. S. Pashinkin, and V. E. Yachmenev, "Temperature dependences of the specific heat and thermodynamic properties of gallium phosphide," Inorganic Materials, vol. 17, pp. 677-680, 1981.

[25] N. N. Sirota, V. V. Novikov, and A. M. Antyukhov, "Heat Capacity and Thermodynamic Functions of (GaAs)x(InAs)1-x Solid Solutions in the 5-300 K Temperature Range," Doklady Akademii Nauk SSSR, vol. 263, no. 1, pp. 96-100, 1982.

[26] N. N. Sirota, A. M. Antyukhov, V. V. Novikov, and A. A. Sidorov, "Temperature Dependence of the Heat Capacity and Characteristic Thermodynamic Functions of the Solid Solutions of $(\mathrm{GaAs})_{x}(\mathrm{InP})_{1-x}$ in the Temperature Range of 5 to $300 \mathrm{~K}$," Doklady Akademii Nauk SSSR, vol. 266, no. 3, pp. 105-108, 1982.

[27] N. N. Sirota, A. M. Antyukhov, V. V. Novikov, and V. A. Fyodorov, "Thermodynamic functions of (InP)x(InAs)1-x from 5 to $300 \mathrm{~K}$," Crystal Research and Technology, vol. 17, no. 3, pp. 279-287, 1982.

[28] J. C. Nipko and C.-K. Loong, "Phonon excitations and related thermal properties of aluminum nitride," Physical Review B-Condensed Matter and Materials Physics, vol. 57, no. 17, pp. 10550-10554, 1998.

[29] J. C. Nipko, C.-K. Loong, C. M. Balkas, and R. F. Davis, "Phonon density of states of bulk gallium nitride," Applied Physics Letters, vol. 73, no. 1, pp. 34-36, 1998.

[30] V. V. Novikov, "The lattice heat capacity of binary compounds in the approximation of three-dimensional debye sublattices," Russian Journal of Physical Chemistry A, vol. 80, no. 9, pp. 14561460, 2006.

[31] A. F. Demidenko and A. K. Maltsev, "Heat capacity of Zinc Telluride in the interval 56-300 ${ }_{o} \mathrm{~K}$," Neorganicheskie Materialy, vol. 5, pp. 158-160, 1969.

[32] N. Vagelatos, D. Wehe, and J. S. King, "Phonon dispersion and phonon densities of states for ZnS and ZnTe," The Journal of Chemical Physics, vol. 60, no. 9, pp. 3613-3618, 1974.

[33] J. C. Irwin and J. Lacombe, "Specific heats of ZnTe, ZnSe, and GaP," Journal of Applied Physics, vol. 45, no. 2, pp. 567-573, 1974.

[34] J. A. Birch, "Heat capacities of ZnS, ZnSe and CdTe below 25K," Journal of Physics C: Solid State Physics, vol. 8, no. 13, article no. 013, pp. 2043-2047, 1975.

[35] J. G. Collins, G. K. White, J. A. Birch, and T. F. Smith, “Thermal expansion of ZnTe and HgTe and heat capacity of $\mathrm{HgTe}$ at low temperatures," Journal of Physics C: Solid State Physics, vol. 13, no. 9, article no. 011, pp. 1649-1656, 1980.

[36] D. N. Talwar, M. Vandevyver, K. Kunc, and M. Zigone, "Lattice dynamics of zinc chalcogenides under compression: Phonon dispersion, mode Grneisen, and thermal expansion," Physical Review B, vol. 24, no. 2, pp. 741-753, 1981.
[37] H. -M. Kagaya and T. Soma, "Temperature dependence of specific heat and Debye temperature of tetrahedral compounds," Physica Status Solidi B, vol. 134, no. 2, pp. K101-K104, 1986.

[38] K. S. Gavrichev, V. N. Guskov, J. H. Greenberg, T. Feltgen, M. Fiederle, and K. W. Benz, "Low-temperature heat capacity of ZnTe," Journal of Chemical Thermodynamics, vol. 34, no. 12, pp. 2041-2047, 2002.

[39] W. T. Berg and J. B. Morrison, "The thermal properties of alkali halide crystals. I. The heat capacities of potassium chloride, potassium bromide, potassium iodide and sodium iodide between 2.8 and $270^{\circ} \mathrm{K}$," in Proceedings of the Royal Society A: Mathematical, Physical and Engineering Sciences, vol. 242, 1231, pp. 467-477, 1957.

[40] M. P. Tosi and F. G. Fumi, "Temperature dependence of the Debye temperatures for the thermodynamic functions of alkali halide crystals," Physical Review, vol. 131, no. 4, pp. 1458-1465, 1963.

[41] I. I. Guseinov and B. A. Mamedov, "Calculation of integer and noninteger $\mathrm{n}$-dimensional debye functions using binomial coefficients and incomplete gamma functions," International Journal of Thermophysics, vol. 28, no. 4, pp. 1420-1426, 2007.

[42] A. E. Dubinov and A. A. Dubinova, "Exact integral-free expressions for the integral Debye functions," Technical Physics Letters, vol. 34, no. 12, pp. 999-1001, 2008.

[43] S. Sönmezoğlu, "Investigation of the entropy and specific heat capacity of GaN using incomplete gamma functions," International Journal of Modern Physics B, vol. 22, no. 30, pp. 5349-5355, 2008.

[44] B. A. Mamedov, E. Eser, H. Koç, and I. M. Askerov, "Accurate evaluation of the specific heat capacity of solids and its application to mgo and zno crystals," International Journal of Thermophysics, vol. 30, no. 3, pp. 1048-1054, 2009.

[45] Y. Yu, H. L. Han, M. J. Wan, T. Cai, and T. Gao, "Structural, vibrational and thermodynamics propertiesof $\mathrm{Zn}$-based semiconductors," Solid State Sciences, vol. 11, no. 8, pp. 1343-1349, 2009.

[46] R. K. Kremer, M. Cardona, R. Lauck, G. Siegle, and A. H. Romero, "Vibrational and thermal properties of $\mathrm{ZnX}(\mathrm{X}=\mathrm{Se}$, Te): Density functional theory (LDA and GGA) versus experiment," Physical Review B - Condensed Matter and Materials Physics, vol. 85, no. 3, Article ID 035208, 2012.

[47] J. A. Beattie, "Six Place Tables of the Debye Energy and Specific Heat Functions," Journal of Mathematics and Physics, vol. 6, no. 1-4, pp. 1-38, 1926.

[48] "Landoldt-börnstein: physikalisch-chemische tabellen," in Die Einsteinschen und Debyeschen Funktionen, W. Roth and K. Scheel, Eds., vol. 1, 1, pp. 702-707, Springer-Verlag, Berlin, Germany, 5th edition, 1927.

[49] P. Harteck, "Landolt-Börnstein: Zahlenwerte und Funktionen aus Physik, Chemie, Astronomie, Geophysik und Technik," in Debye-Funktionen, vol. 67, pp. 767-768, 23 edition, 1955.

[50] R. Pässler, "Representative hybrid model used for analyses of heat capacities of group-IV, III-V, and II-VI materials," Physica Status Solidi (B) Basic Research, vol. 248, no. 4, pp. 904-920, 2011.

[51] A. A. Maradudin and R. F. Wallis, "Lattice-dynamical calculation of the surface specific heat of a crystal at low temperatures," Physical Review, vol. 148, no. 2, pp. 945-961, 1966.

[52] A. A. Maradudin, E. W. Montroll, G. H. Weiss, and I. P. Ipatova, "Theory of Lattice Dynamics in the Harmonic Approximation," in Solid State Physics, H. E. Ehrenreich et al., Ed., 3, p. viii+319, 
Academic Press, New York-London, New York, NY, USA, 2nd edition, 1971.

[53] C. Lee and X. Gonze, "Ab initio calculation of the thermodynamic properties and atomic temperature factors of $\mathrm{SiO} 2 \alpha$ quartz and stishovite," Physical Review B, vol. 51, no. 13, pp. 8610-8613, 1995.

[54] R. Pässler, "Exponential series representation for heat capacities of semiconductors and wide-bandgap materials," Physica Status Solidi (B) Basic Research, vol. 245, no. 6, pp. 1133-1146, 2008.

[55] W. Xue, Y. Yu, Y. Zhao, H. Han, and T. Gao, "First principles calculations of the electronic, dynamical, and thermodynamic properties of the rocksalt $\mathrm{ScX}(\mathrm{X}=\mathrm{N}, \mathrm{P}, \mathrm{As}, \mathrm{Sb})$," Computational Materials Science, vol. 45, no. 4, pp. 1025-1030, 2009.

[56] R. Pässler, "Basic moments of phonon density of states spectra and characteristic phonon temperatures of group IV, III-V, and II-VI materials," Journal of Applied Physics, vol. 101, no. 9, Article ID 093513, 2007.

[57] H. Neumann, G. Kühn, and W. Möller, "Heat Capacity and Lattice Anharmonicity in Cu-III-VI2 Chalcopyrite Compounds," physica status solidi (b), vol. 144, no. 2, pp. 565-573, 1987.

[58] W. Nernst and F. A. Lindemann, "Spezifische Wärme und Quantentheorie," Zeitschrift für Elektrochemie, vol. 17, pp. 817827, 1911.

[59] R. Pässler, "Characteristic non-Debye heat capacity formula applied to GaN and ZnO," Journal of Applied Physics, vol. 110, no. 4, Article ID 043530, 2011.

[60] A. Sommerfeld, "Zur Elektronentheorie der Metalle auf Grund der Fermischen Statistik. I. Teil: Allgemeines, Strömungs- und Austrittsvorgänge," Zeitschrift für Physik, vol. 47, no. 1-2, pp. 132, 1928.

[61] N. Pearlman and P. H. Keesom, "The atomic heat of silicon below 100॰K," Physical Review, vol. 88, no. 2, pp. 398-405, 1952.

[62] C. A. Bryant and P. H. Keesom, "Low-temperature specific heat of germanium," Physical Review, vol. 124, no. 3, pp. 698-700, 1961.

[63] M. Wagner-Reetz, D. Kasinathan, W. Schnelle et al., "Phonondrag effect in FeGa3," Physical Review B - Condensed Matter and Materials Physics, vol. 90, no. 19, Article ID 195206, 2014.

[64] T. H. K. Barron and J. A. Morrison, "The Thermal Properties of Alkali Halide Crystals. III. The Inversion of the Heat Capacity," Proceedings of the Royal Society A: Mathematical, Physical and Engineering Sciences, vol. 256, no. 1286, pp. 427-439, 1960.

[65] J. C. Holste, "Specific heats of GaSb, GaAs, InSb, InAs, Bi, Cd, Sn, and Zn below 30 K," Physical Review B, vol. 6, no. 6, pp. 2495-2497, 1972.

[66] B. Hennion, F. Moussa, G. Pepy, and K. Kunc, "Normal modes of vibrations in ZnSe," Physics Letters A, vol. 36, no. 5, pp. 376-378, 1971.

[67] H. Bilz and W. Kress, Phonon Dispersion Relations in Insulators, vol. 10 of Springer Series in Solid-State Sciences, Springer, Heidelberg, Berlin, Germany, 1979.

[68] A. Einstein, "Die Plancksche Theorie der Strahlung und die Theorie der spezifischen Wärme," Annalen der Physik, vol. 327, no. 1, pp. 180-190, 1906.

[69] H. Thirring, "Zur Theorie der Raumgitterschwingungen und der spezifischen $\mathrm{W} \Sigma$ rme fester $\mathrm{K} \div$ rper," Physikalische Zeitschrift, vol. 14, pp. 876-873, 1913.

[70] E. Gmelin, "Thermal Properties of Alkaline Earth Oxides: II. Analysis of Experimental Results for $\mathrm{MgO}, \mathrm{CaO}, \mathrm{SrO}$, and $\mathrm{BaO}$," Zeitschrift fur Naturforschung - Section A Journal of Physical Sciences, vol. 25, no. 6, pp. 887-893, 1970.
[71] A. P. Rusakov, Y. K. Vekilov, and A. E. Kadyshevich, "Specific Heats of CdTe and HgTe and their Vibrational Frequency Spectra," Fizika Tverdogo Tela, vol. 12, no. 11, pp. 3238-3243, 1970.

[72] R. Pässler, "Moments of phonon density of states spectra and characteristic phonon temperatures of wide band gap materials," Physica Status Solidi (B) Basic Research, vol. 243, no. 12, pp. 2719-2727, 2006.

[73] H. Wagini, "Systematik charakteristischer Temperaturen von Halbleitern mit Zinkblende-Gitter," Zeitschrift fur Naturforschung - Section A Journal of Physical Sciences, vol. 22, no. 7, pp. 1135-1136, 1967.

[74] B. H. Lee, "Elastic constants of ZnTe and ZnSe between 77o300॰K," Journal of Applied Physics, vol. 41, no. 7, pp. 2984-2987, 1970.

[75] J. K. D. Verma and M. D. Aggarwal, "An averaging method for the shear modulus and Debye temperatures of cubic solids," Journal of Applied Physics, vol. 46, no. 7, pp. 2841-2844, 1975.

[76] D. Singh and Y. P. Varshni, "Debye temperatures for hexagonal crystals," Physical Review B, vol. 24, no. 8, pp. 4340-4347, 1981.

[77] H. Siethoff and K. Ahlborn, "The Dependence of the Debye Temperature on the Elastic Constants," Physica Status Solidi (B), vol. 190, no. 1, pp. 179-191, 1995.

[78] A. S. Pashinkin and A. S. Malkova, "Heat capacity of Zinc Selenide," Russian Journal of Physical Chemistry A, vol. 77, no. 12, pp. 2068-2069, 2003.

[79] N. N. Sirota, Z. K. Petrova, and T. D. Sokolovsky, "Heat Capacity of Zinc Selenide over the Temperature Range 4.2 to $300 \mathrm{~K}$," Doklady Akademii Nauk Belorusi, vol. 24, no. 3, pp. 214-217, 1980.

[80] G. G. Gadzhiev, Sh. M. Ismailow, and A. I. Dadashev, “Thermal Properties of AIIBVI-Based Ceramics," High Temperature, vol. 31, no. 3, pp. 350-354, 1993.

[81] I. Hurtado and D. Neuschütz, Landolt-Börnstein: Numerical Data and Functional Relationships in Science and Technology, Group IV, (Thermodynamic Properties of Inorganic Materials), vol. 19 of Subvolume A, Parts 1-4, Springer-Verlag, Berlin, Heidelberg, 1999.

[82] K. S. Gavrichev, G. A. Sharpataya, V. N. Guskov et al., “Thermodynamic properties of ZnTe in the temperature range 15-925 K," Physica Status Solidi (B) Basic Research, vol. 229, no. 1, pp. 133-135, 2002.

[83] T. H. K. Barron and J. A. Morrison, "On the specific heat of solids at low temperatures," Canadian Journal of Physics, vol. 35, no. 7, pp. 799-810, 1957.

[84] M. Cardona, R. K. Kremer, R. Lauck, G. Siegle, J. Serrano, and A. H. Romero, "Heat capacity of PbS: Isotope effects," Physical Review B - Condensed Matter and Materials Physics, vol. 76, no. 7, Article ID 075211, 2007.

[85] F. Kelemen, E. Cruceanu, and D. Niculescu, "Untersuchung einiger thermischer Eigenschaften der Verbindungen HgSe, HgTe und ZnTe," physica status solidi (b), vol. 11, no. 2, pp. 865872, 1965.

[86] L. Guo, G. Hu, W.-J. Feng, and S.-T. Zhang, "Structural, elastic, electronic and optical properties of zinc-blende MTe (M=Zn/Mg)," Wuli Huaxue Xuebao/ Acta Physico-Chimica Sinica, vol. 29, no. 5, pp. 929-936, 2013.

[87] A. K. Kushwaha, "Phonon spectrum and thermal properties of semiconducting compounds $\mathrm{ZnS}$ and ZnSe," Physica B: Condensed Matter, vol. 405, no. 6, pp. 1638-1642, 2010. 
[88] U. C. Boehnke, G. Kühn, F. I. Frolova, I. E. Paukov, and H. Neumann, "Heat capacity of LiInTe2," Journal of Thermal Analysis, vol. 33, no. 1, pp. 205-209, 1988.

[89] K. Kunc, M. Balkanski, and M. A. Nusimovici, "Lattice dynamics of several $\mathrm{a}^{N} \mathrm{~B}^{8-N}$ compounds having the zincblende structure. II. Numerical Calculations," Physica Status Solidi B, vol. 72, no. 1, pp. 249-254, 1975.

[90] M. Abramowitz and I. A. Stegun, "Pocketbook of mathematical functions, Verlag Harri Deutsch," Thun-Frankfurt/Main, 1984.

[91] H. J. Schell, "Unendliche Reihen," in Mathematik für Ingenieure, Naturwissenschaftler, Ökonomen und Landwirte, K. Manteuffel, Ed., vol. 3, BSB B. G. Teubner Verlagsgesellschaft, Leipzig, Germany, 1st edition, 1974. 

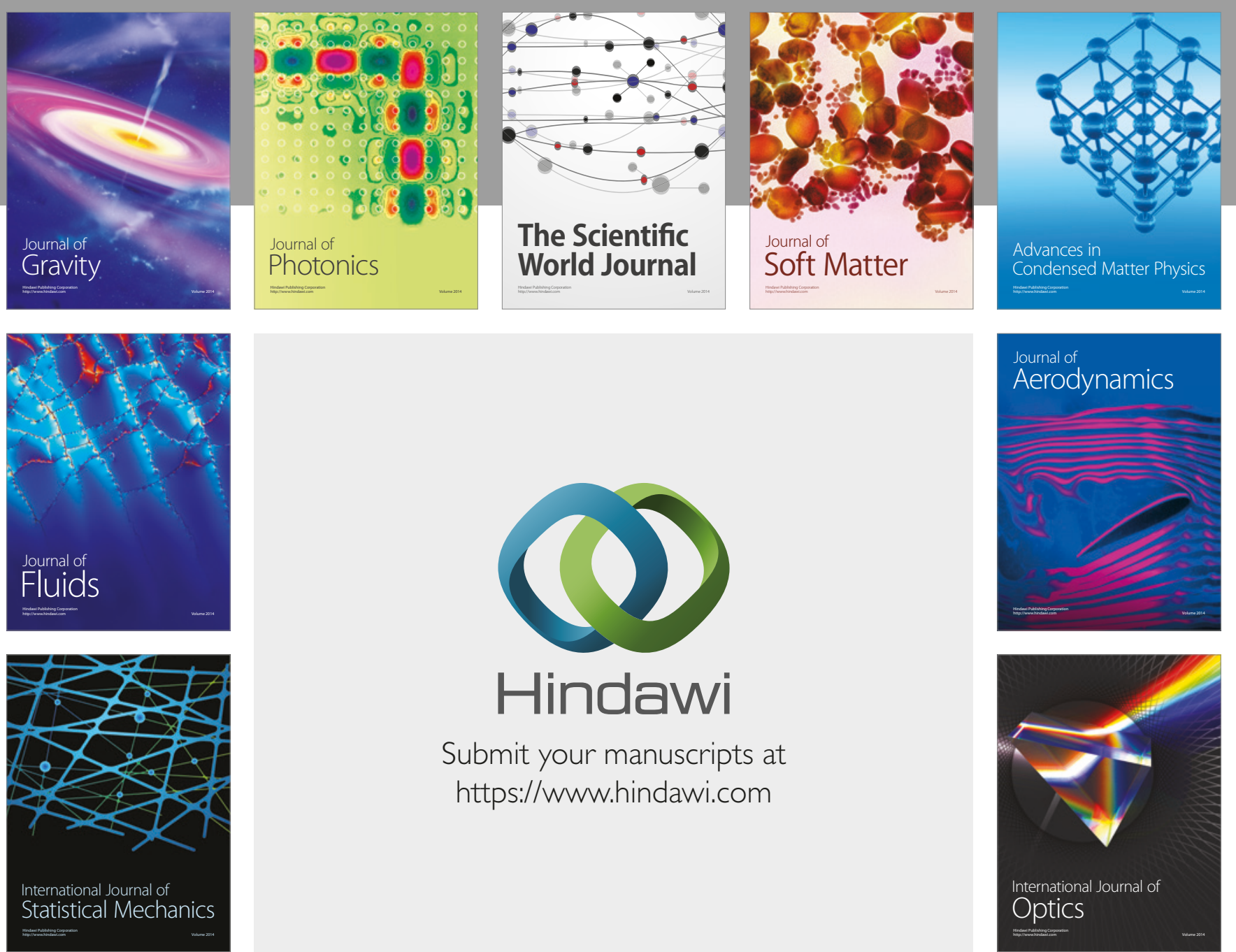

Submit your manuscripts at

https://www.hindawi.com
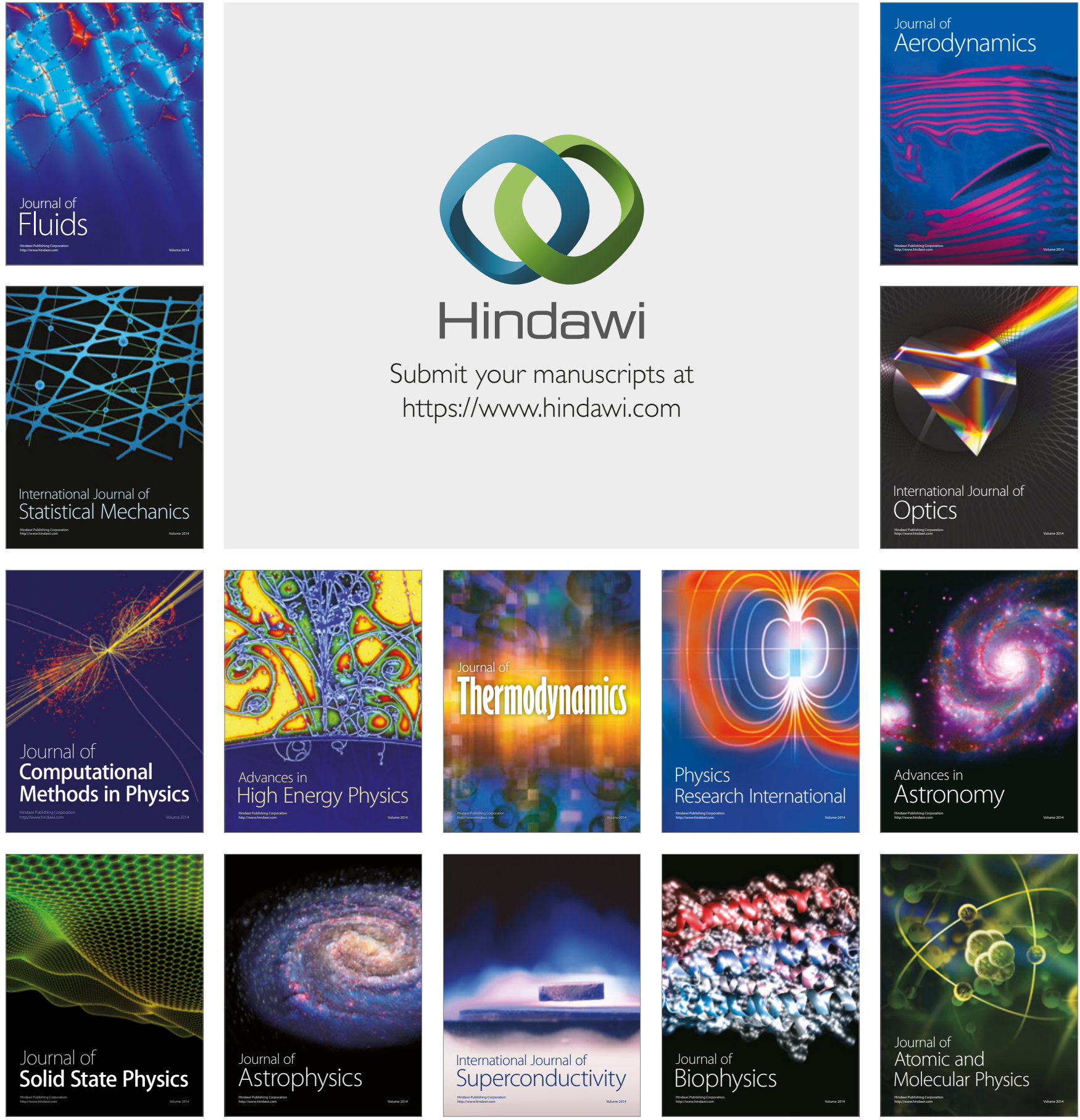